\title{
An Ecosystem-Based Approach to Habitat Restoration Projects with Emphasis on Salmonids in the Columbia River Estuary
}

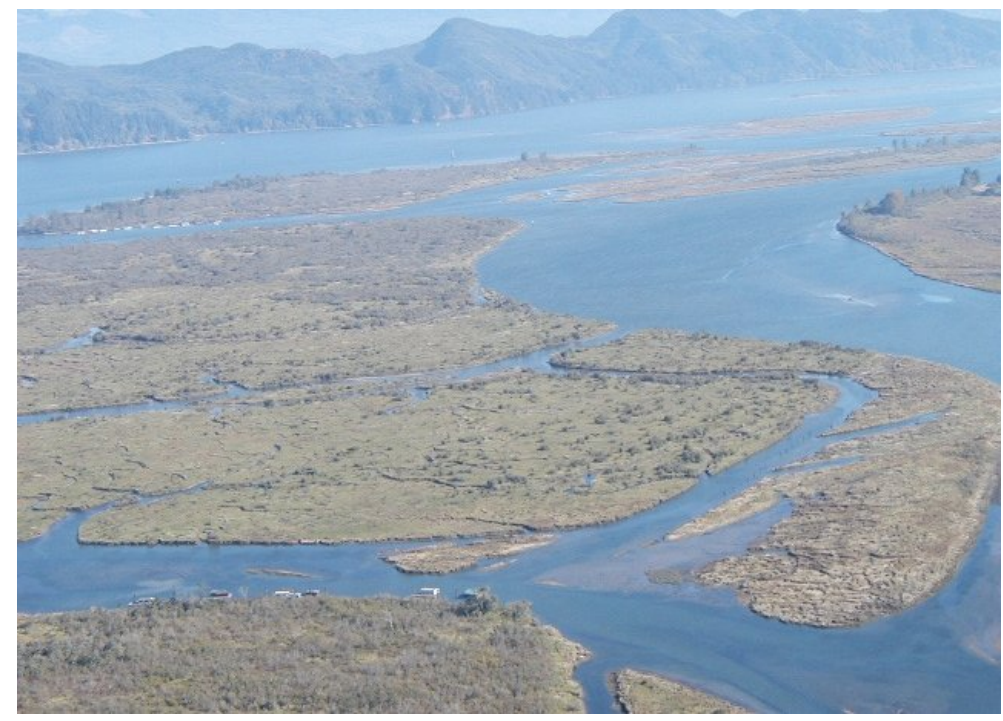

FINAL REPORT

November 2003

Prepared for the U.S. Department of Energy under Contract DE-AC06-76RL01830

\section{Prepared by:}

Pacific Northwest National Laboratory

Columbia River Estuary Study Taskforce

Lower Columbia River Estuary Partnership

Bonneville Power Administration

U. S. Army Corps of Engineers, Portland District 


\title{
DISCLAIMER
}

This report was prepared as an account of work sponsored by an agency of the United States Government. Neither the United States Government nor any agency thereof, nor Battelle Memorial Institute, nor any of their employees, makes any warranty, express or implied, or assumes any legal liability or responsibility for the accuracy, completeness, or usefulness of any information, apparatus, product, or process disclosed, or represents that its use would not infringe privately owned rights. Reference herein to any specific commercial product, process, or service by trade name, trademark, manufacturer, or otherwise does not necessarily constitute or imply its endorsement, recommendation, or favoring by the United States Government or any agency thereof, or Battelle Memorial Institute. The views and opinions of authors expressed herein do not necessarily state or reflect those of the United States Government or any agency thereof.

\author{
PACIFIC NORTHWEST NATIONAL LABORATORY \\ operated by \\ BATTELLE \\ for the \\ UNITED STATES DEPARTMENT OF ENERGY \\ under Contract DE-AC06-76RL01830
}

Cover Photo: Cathlamet Bay, courtesy of CREST. 


\section{An Ecosystem-Based Approach to Habitat Restoration Projects with Emphasis on Salmonids in the Columbia River Estuary}
G. E. Johnson ${ }^{(a)}$
B. D. Ebberts (d)
R. M. Thom ${ }^{(a)}$
N. M. Ricci(e)
A. H. Whiting(b)
J. A. Southard(a)
G. B. Sutherland(c)
J.D. Wilcox ${ }^{(e)}$
T. Berquam (d)

FINAL REPORT

November 2003

Prepared by:

Pacific Northwest National Laboratory

Columbia River Estuary Study Taskforce

Lower Columbia River Estuary Partnership

Bonneville Power Administration

U. S. Army Corps of Engineers, Portland District

\footnotetext{
(a) Pacific Northwest National Laboratory

(b) Columbia River Estuary Study Taskforce

(c) Lower Columbia River Estuary Partnership

(d) U. S. Army Corps of Engineers, Portland District

(e) Bonneville Power Administration
} 



\section{Executive Summary}

Habitat restoration in the Columbia River estuary (CRE) is an important off-site mitigation action in the 2000 Biological Opinion (BiOp), an operation of the Federal Columbia River Power System. The CRE, defined as the tidally influenced stretch of river from the mouth to Bonneville Dam 146 miles upstream, is part of the migration pathway for anadromous fish in the Columbia Basin, including salmon listed under the Endangered Species Act (ESA). Salmon in various stages of life, from fry to adults, use tidal channels and wetlands in the CRE to feed, find refuge from predators, and transition physiologically from freshwater to saltwater. Over the last 100 years, however, the area of some wetland habitats has decreased by as much as $70 \%$ because of dike and levee building, flow regulation, and other activities. In response to the decline in available habitat, the BiOp's Reasonable and Prudent Alternative (RPA) included mandates to “... develop a plan addressing the habitat needs of juvenile salmon and steelhead in the estuary” (RPA Action 159) and “...develop and implement an estuary restoration program with a goal of protecting and enhancing 10,000 acres of tidal wetlands and other key habitats...” (RPA Action 160). To meet Action 159 and support Action 160, this document develops a science-based approach designed to improve ecosystem functions through habitat restoration activities in the CRE.

The CRE habitat restoration program's goal and principles focus on habitat restoration projects in an ecosystem context. Since restoration of an entire ecosystem is not generally practical, individual habitat restoration projects have the greatest likelihood of success when they are implemented with an ecosystem perspective. The program's goal is:

Implementation of well-coordinated, scientifically sound projects designed to enhance, protect, conserve, restore, and create 10,000 acres of tidal wetlands and other key habitats to aid rebuilding of ESA-listed salmon populations and native species using the CRE.

The program's underlying principles are: 1) projects are founded on the best available ecological restoration science, implemented in an ecosystem context, and developed with the intent to restore relevant ecological processes; 2) projects incorporate adaptive management practices with testable hypotheses to track ecological responses to a given restoration effort; and 3) projects are implemented in a coordinated, open process and scientific results from monitoring and evaluation are communicated widely and readily accessible. With this goal and these principles in mind, we developed an approach for CRE habitat restoration.

The intent of this document is to provide a scientific basis and implementation guidelines for a habitat restoration program designed to improve ecosystem functions and enhance juvenile salmonid survival in the CRE. The approach advocated in this document may be summarized in the following sequence of steps.

1. Describe the fundamentals of restoration science. The approach draws from the disciplines of restoration ecology, landscape ecology, and conservation biology. Restoration fundamentals include five basic restoration strategies: conservation, creation, enhancement, restoration, and protection (see main text for definitions). The usefulness of a given restoration strategy depends on the level of disturbance at the landscape and local scales. The CRE can be classified as moderately disturbed on the landscape scale because of altered hydrology, loss of tidal wetlands, 
and altered bathymetry. At the local scale, the level of anthropogenic or natural disturbance varies. Restoration success is dependent on recovering and sustaining ecosystem functions and processes.

2. Determine usage of CRE habitats by salmonid life history type, i.e., determine which habitats are most important and why. It is essential for strategic restoration planning to know how fish of various life history types use CRE habitats in space and time in order to identify the habitat attributes juvenile salmon need (capacity) and to establish what habitats are important to what types of fish (life history type). Briefly, literature on salmonid usage reviewed for this document revealed: a) life history diversity is reduced compared to historical levels; b) hatchery fish predominate, therefore timing and abundance are influenced by hatchery release practices; and, c) most juvenile Chinook salmon captured in recent surveys in CRE wetland habitats were from watersheds in the lower Columbia River. However, there are several key uncertainties in our knowledge of salmonid usage of CRE habitats yet to be resolved (see below for suggested next steps for the stakeholders to implement).

3. Determine what CRE habitats have been lost relative to historical conditions (pre-development in 1900s). A habitat change analysis (1890 vs. 1992 habitat maps for the lower 46 river miles) determined tidal swamp and tidal marsh habitats have suffered the largest relative declines. This means that opportunity for juvenile salmonids to use these areas, i.e., accessibility, has been diminished. This habitat decline is due mostly to diking, filling, and flow regulation. These actions effectively eliminated the exchange of water, materials, and organisms between the larger aquatic system and the adjacent, shallowly vegetated habitats, although some exchange occurred through tide gates. Exchange is important not only for the access and use of these habitats by fish, but also for maintenance of habitat-forming processes such as sedimentation and erosion. By restoring tidal exchange, these processes are re-established, and the habitats can develop naturally as well as provide their functions to the ecosystem.

4. Identify and prioritize restoration strategies for the CRE and establish a reasonable future condition, given constraints on the system (e.g., flow regulation). This document suggests priorities for restoration strategies depending on the degree of disturbance in bathymetry at the landscape scale and in tidal exchange at the local scale. Regarding a "reasonable future condition”, historical conditions may be assumed to represent optimal habitat conditions for a particular site, but it must be noted that present-day conditions of the CRE may constrain the type, size, and functionality of said habitat conditions. Therefore, optimal habitat conditions for a site under present-day CRE conditions may differ from optimal historical conditions. In any event, a return to a more "normative" state than is currently present may be advantageous.

5. Determine what specific habitats can be restored and where, i.e., develop an inventory of possible actions. This step integrates and applies knowledge gained in the previous four steps. It is intended to provide specific guidance to restoration planners and requires a high level of detail and analysis from habitat mapping and inventory efforts. Although this step was beyond the scope of this document, it will be important for others to accomplish in the near future.

6. Implement locally supported and scientifically based restoration projects. This document includes guidelines to implement restoration projects. This involves a description of specific types of projects that meet the recommended CRE restoration strategies developed above. We 
proposed new project selection guidelines that build on existing work by the Estuary Partnership. The main phases of a restoration project (planning, construction, effectiveness monitoring, data management and dissemination, and adaptive management) are explained to provide a framework for restoration project planners.

7. Monitor actions using standardized protocols and apply the results to adaptively manage future restoration actions. Restoration projects should be treated as "experiments" with reference sites also included in the monitoring scheme. Then, the results of each experiment can be evaluated and future or ongoing restoration projects revised as necessary. A process to coordinate, monitor performance, collect and disseminate data, and adaptively manage multiple projects is described in the document. These monitoring activities will be consistent with the estuary/plume portion of the regional Research, Monitoring, and Evaluation Plan for the BiOp.

The stepwise approach to CRE habitat restoration outlined above is somewhat general and broad because the available scientific information is incomplete, e.g., juvenile salmon usage of various CRE wetland habitats. As new data become available, a more specific, detailed plan than was possible here can be produced as an outgrowth of this document. Suggested next steps ${ }^{1}$ for the stakeholders to take to develop a detailed CRE habitat restoration plan are to:

1. Organize and empower a coordinating body to provide technical and policy oversight of habitat restoration activities in the CRE. The coordinating body would evaluate proposed projects, seek support and funding for projects, discuss progress and new developments, review monitoring and restoration activities, assess project effectiveness, share data, and disseminate information.

2. Resolve the critical uncertainties concerning salmon habitat requirements in the CRE. To reiterate, these uncertainties (see Chapter 2.3, Juvenile Salmon Usage) include: the relationship between life history strategies and habitat requirements; juvenile usage and basic ecology of the tidal freshwater (RM 46-146) subsystem; linkages between biological and physical processes in various habitat types; the landscape qualities of preferred habitats; growth and survival rates for various life history types in relation to habitat attributes; habitat forming processes for key habitat types; and food web interactions.

3. Intensify the ongoing habitat mapping effort, and apply the results to develop a comprehensive, unified, and quantified habitat inventory for the CRE.

4. Finalize the proposed project selection guidelines and list specific restoration projects and potential sites.

5. Prioritize restoration projects that promote the long-term sustainability of ecosystem function and structure at the landscape scale wherever possible.

\footnotetext{
${ }^{1}$ The next steps incorporate review comments provided by J. Marshall (USFWS), the Independent Scientific Review Panel, and NOAA Fisheries.
} 
6. Consider changing from RM 0-46 to RM 0-146 the credit given for projects under BiOp RPA Action 160.

7. Establish a trust fund for CRE habitat restoration projects.

8. Perform effectiveness monitoring for implemented projects and develop methods to evaluate cumulative effects of multiple projects.

9. Establish a data system for CRE habitat restoration.

10. Embrace a true adaptive management process as part of CRE habitat restoration efforts.

11. Apply the restoration approach and implementation guidelines in this document and as new information becomes available, institute follow-up work to develop a project implementation guidebook and a revised strategic plan for CRE habitat restoration.

In conclusion, this document provides a scientific basis and implementation guidelines for a habitat restoration program designed to improve ecosystem functions and enhance juvenile salmonid survival in the CRE. As more experience is gained with CRE habitat restoration and scientific uncertainties are resolved, this document should be used as a basis for a detailed habitat restoration plan that specifically addresses 1) which habitat types offer the greatest ecological benefit to salmon, 2) the location of potential sites that if restored would likely provide these habitat types, and 3) how and when the restoration work should be done. This document supports the use of adaptive management so that all elements of salmonid habitat restoration actions in the CRE are under continual evaluation and revision at both the project and program levels. Lessons learned from current and proposed habitat restoration projects need to be applied to all future work, such as the Estuary Partnership's habitat restoration program and the U.S. Army Corps of Engineers' General Investigation Study for the CRE, to ensure the most effective use of resources and the best possible long term environment for salmonid growth and survival in the CRE. 


\section{Preface}

The Bonneville Power Administration (BPA), in coordination with the U.S. Army Corps of Engineers (COE) and the National Oceanic and Atmospheric Administration (NOAA), originated this project (BPA Project No. 2002-076-000; Contract No. 652, Release No. 24). Their intent was to address Reasonable and Prudent Alternative (RPA) Action 159 of the 2000 National Marine Fisheries Service Biological Opinion (BiOp) on operation of the Federal Columbia River Power System (FCRPS). Other coordinated efforts underway addressing Action 159 include the COE's General Investigations Study for the Columbia River Estuary CRE, the Lower Columbia River Estuary Partnership's (Estuary Partnership) sub-basin planning and habitat mapping projects, and the BPA/COE's research, monitoring, and evaluation plan for the estuary and plume.

The intent of this document is to provide a scientific basis and implementation guidelines for a habitat restoration program designed to improve ecosystem functions and enhance juvenile salmonid survival in the Columbia River estuary (CRE). The document does not address economic, social, or political aspects of habitat restoration in the CRE. The focus here is on habitat for listed salmon, although the ecosystem-based approach necessarily affects other species as well. Salmon habitat restoration is best undertaken within the context of other biota and physical processes using an ecosystem perspective. The anticipated audience includes entities responsible for, interested in, or affected by habitat restoration in the CRE. Timeframes to apply this document extend from the immediate (2003-2004) to the near-term (2005-2006) to the long-term (2007 and beyond). We anticipate and encourage that the document be revised as new knowledge and experience are attained.

A team comprised of staff from the Columbia River Estuary Study Taskforce (CREST), the Estuary Partnership, and Pacific Northwest National Laboratory (PNNL) wrote this document. As the responsible Action Agencies, the BPA and the COE provided oversight. The Estuary Partnership's Science Work Group, the Independent Scientific Review Panel, NOAA Fisheries staff, and Northwest Power and Conservation Council (NPCC) staff reviewed drafts of this document. Extensive efforts were made to ensure a sound technical and policy basis and to solicit input from all interested parties.

The reader should understand the BPA is only responsible for "protection and enhancement" projects in the Columbia River estuary under the FCRPS BiOp. Similarly, the COE is only responsible for "restoration" projects under their authorities. The actions and recommendations in this document are intended to guide restoration activities, and shall not bind or commit the BPA or COE to any particular project or course of action. 


\section{Acknowledgments}

We gratefully acknowledge contributions to this document by P. Adamus (Oregon State University), H. Diefenderfer (PNNL), N. Evans (PNNL), C. Furey (BPA), T. Gilbride (PNNL), K. Larsen (COE), D. Marriott (Estuary Partnership), A. Ruger (BPA), J. Slater (PNNL), C. Tortorici (NOAA Fisheries), M. Van Ess (CREST), S. Waste (NPCC), B. Willis (COE), and the Estuary Partnership's Science Work Group and staff. In particular, review comments by J. Marshall (USFWS), the Independent Scientific Review Panel, and NOAA Fisheries were instrumental in shaping the discussion of next steps for Columbia River Estuary habitat restoration. 


\section{Contents}

Executive Summary ….....................................................................................

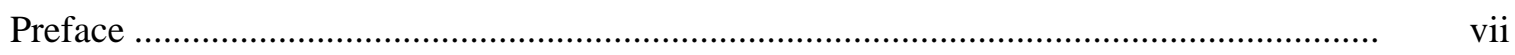

Acknowledgments............................................................................................ ix

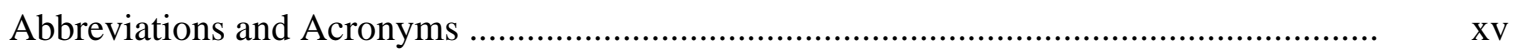

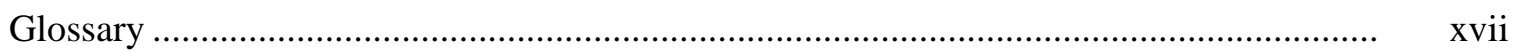

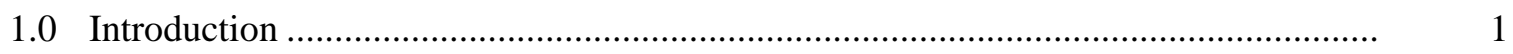

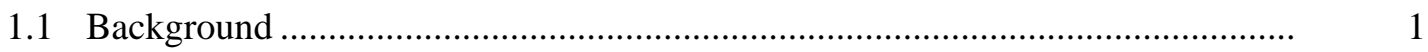

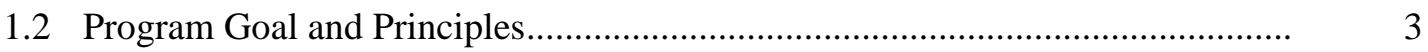

1.3 Study Objectives and Tasks ......................................................................... 4

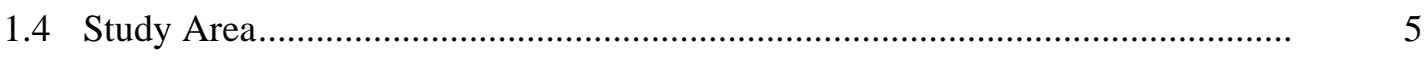

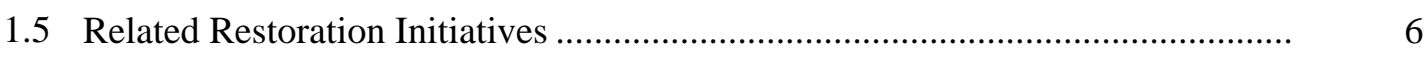

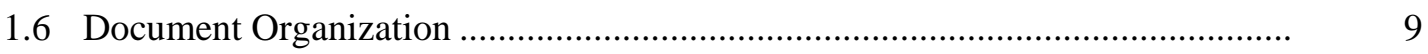

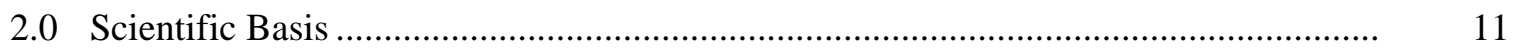

2.1 Fundamentals of Ecological Restoration............................................................. 11

2.1.1 General Model for Ecosystem State .......................................................... 11

2.1.2 Role of Landscape Ecology ..................................................................... 11

2.1.3 Generic Restoration Strategies .............................................................. 11

2.1.4 Influence of Disturbances.................................................................... 11

2.2 Conceptual Ecosystem Model ........................................................................... 20

2.2.1 Background and Objective of a Conceptual Model ................................ 20

2.2.2 A Conceptual Model for the CRE ........................................................... 21

2.3 Juvenile Salmon Usage of the CRE ….................................................................... 23

2.3.1 CRE Salmonid Species......................................................................... 23

2.3.2 Life History Strategies ................................................................................ 24

2.3.3 Temporal Distribution and Residence Time ........................................... $\quad 24$

2.3.4 Spatial Distribution and Physical Habitat Characteristics.......................... 25

2.3.5 Prey Resources .................................................................................... 26

2.3.6 Summary of Habitat Requirements and Uncertainties ............................ 27

2.4 Habitat Changes in the Columbia River Estuary............................................... 28

2.4.1 Habitat Types ............................................................................. 29

2.4.2 Subsystems and Subareas .............................................................. 29 
2.4.3 Summary of Habitat Changes................................................................. $\quad 40$

2.5 Restoration Strategies for the CRE ................................................................. 42

2.6 The Scientific Basis for CRE Restoration: Results of the Analysis ..................... 47

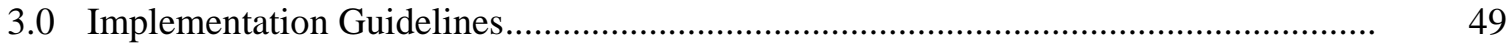

3.1 Types of On-the-Ground Projects to Implement CRE Restoration Strategies ..... 49

3.1.1 Conservation................................................................................... 51

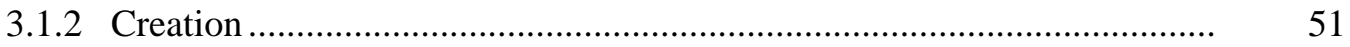

3.1.3 Enhancement ..................................................................................... 51

3.1.4 Restoration ................................................................................... 51

3.1.5 Protection ..................................................................................... 52

3.1.6 Brief Summary of Selected CRE Restoration Projects ............................ 52

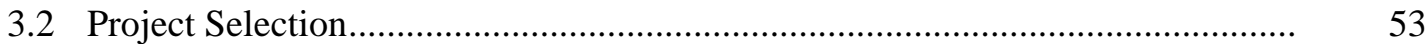

3.2.1 Review of Prioritization Approaches ...................................................... 53

3.2.2 Multi-Criteria Methods ........................................................................... $\quad 55$

3.2.3 Relevance to the CRE ...................................................................... 55

3.2.4 General Prioritization Method for CRE Restoration Projects .................. 56

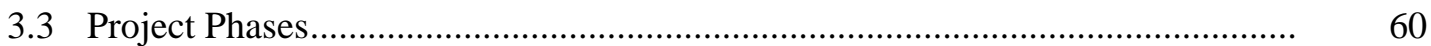

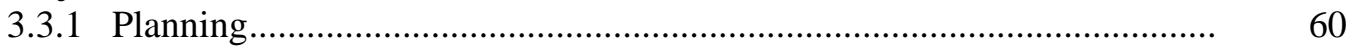

3.3.2 Implementation................................................................................. 63

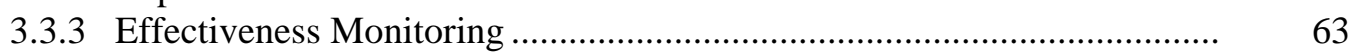

3.3.4 Data Management and Dissemination..................................................... 65

3.3.5 Adaptive Management .................................................................... 66

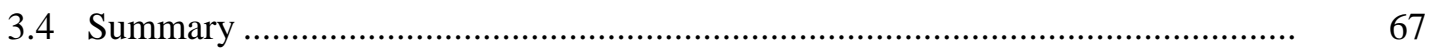

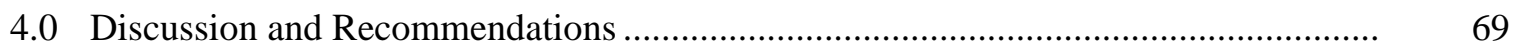

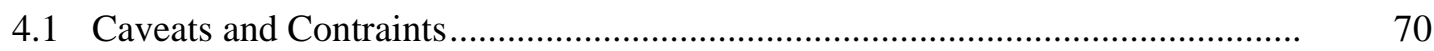

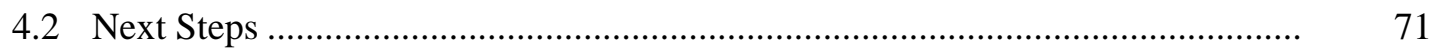

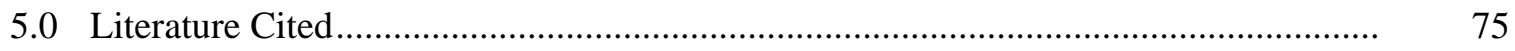

Appendix A - Related Ecosystem Restoration Initiatives ................................................. A.1

Appendix B - Conceptual Ecosystem Model for the CRE ................................................. B.1 


\section{Figures}

1.1 Columbia River Estuary Study Area from the Mouth to Bonneville Dam................. 2

1.2 Satellite Photograph of the Lower Portion of the Columbia River Estuary ................... 6

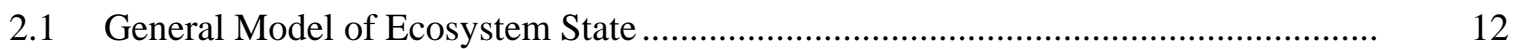

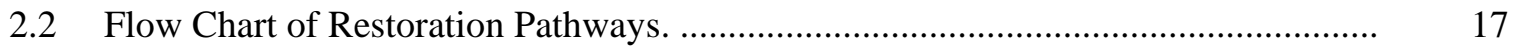

2.3 Restoration Strategies for Estuarine Systems Relative to Disturbance Levels at the Site and in the Landscape....................................................................................... 19

2.4 Conceptual Model of Habitat Forming Processes for the CRE. ................................ 22

2.5 Conceptual Model for Juvenile Salmon Growth and Survival. ................................. 22

2.6 Columbia Estuary Subsystem with Seven Subareas ................................................ 30

2.7 Habitat Maps of the Entrance Subarea ......................................................................... 31

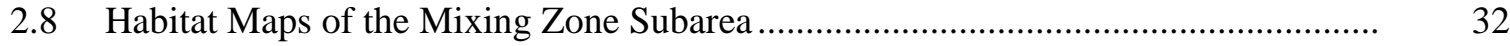

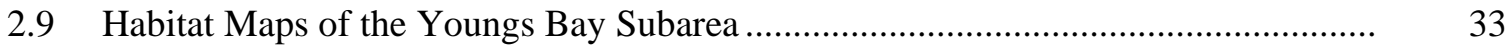

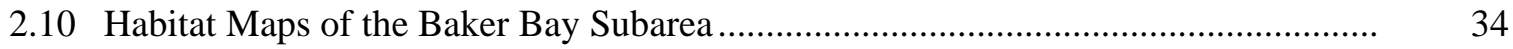

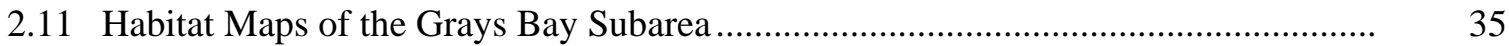

2.12 Habitat Maps of the Cathlamet Bay Subarea ............................................................... 36

2.13 Habitat Maps of the Upper Estuary Subarea................................................................ 37

2.14 Habitat Maps of the Tidal Freshwater Subsystem (RM 46-105) ................................. 38

2.15 Habitat Maps of the Tidal Freshwater Subsystem (RM 105-146) .............................. 37

2.16 Changes in Area Covered by Aquatic Habitat Types in the Estuarine Subsystem (RM 0-46) .............................................................................. 40

2.17 Historical and Present-Day Coverage of Five Habitat Types within the Seven Subareas of the Estuarine Subsystem (RM 0-46)

2.18 Matrix of Habitat Restoration Strategies Appropriate for Increasing Degrees of Disturbance by Subsystem and Subarea

2.19 The Estuary Subareas and the Tidal Freshwater Subsytem Relative to Disturbances in Tidal Exchange and Bathymetry 


\section{Tables}

1.1 Brief Summaries of Major Regional Habitat Restoration Programs in the United States

2.1 Sample list of selected structural and related functional attributes for

2.2 Explanations of Certain Landscape Ecology Concepts............................................. 14

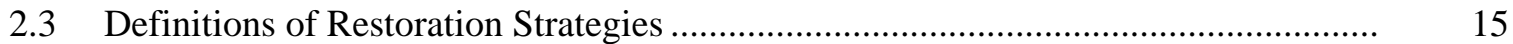

2.4 Technical Recovery Team, Evolutionarily Significant Unit, and Endangered Species Act Listing under the Federal Columbia River Power System Biological Opinion

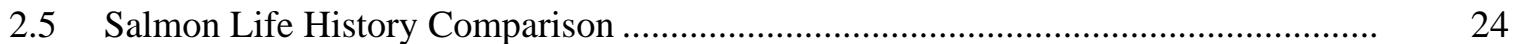

2.6 Physical Habitat Requirements for Juvenile Salmon in the CRE .............................. 26

2.7 Summary of Habitat Mapping Projects for the CRE …............................................ 29

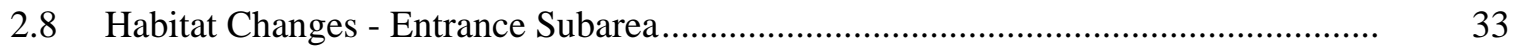

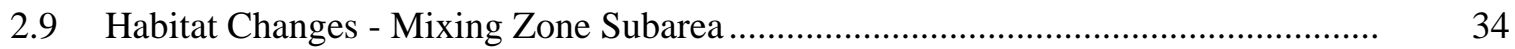

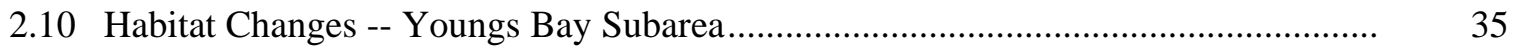

2.11 Habitat Changes - Baker Bay Subarea ....................................................................... 36

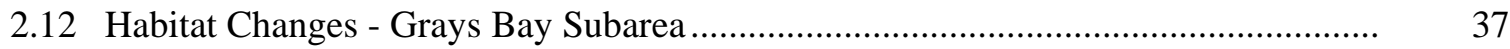

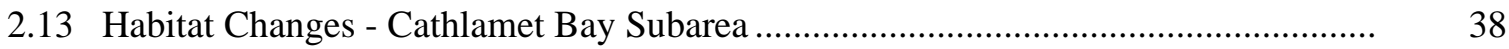

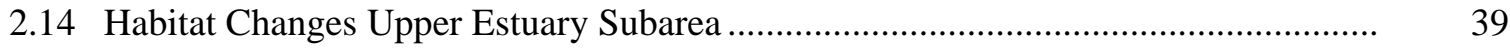

2.15 Summary Totals for the Habitat Changes Presented in Tables 2.8 - 2.14 for the Estuarine Subsystems

2.16 Associated Alterations and Metrics Indicating the Degree of Disturbance for Three Controlling Factors in the CRE

2.17 Summary of Controlling Factor Disturbance Level within each Subarea of the Estuarine and Tidal Freshwater Subsystems.

2.18 Recommended CRE Restoration Strategies by Subarea based on the Disturbance Matrix. 
3.1 Examples of Project Types and Targeted Ecosystem Benefit by Restoration Strategy for the CRE

3.2 Selected Completed and Ongoing Restoration Projects in the CRE.

3.3 Category of metrics used to assess effects of shoreline armoring on salmonid growth and survival.

3.4 Project Selection Guidelines and Scorecard

3.5 An Adaptive Management Framework for Restoration Project Phases, Activities, Suggested Tools, and Pertinent Sections in the Plan 


\section{Abbreviations and Acronyms}

\begin{tabular}{|c|c|}
\hline $\mathrm{BiOp}$ & Biological Opinion \\
\hline BPA & Bonneville Power Administration \\
\hline CAP & Continuing Authorities Program \\
\hline $\mathrm{COE}$ & U.S. Army Corps of Engineers \\
\hline CERP & Comprehensive Everglades Restoration Plan \\
\hline CRE - & Columbia River estuary \\
\hline CREDDP & Columbia River Estuary Data Development Program \\
\hline CREST & Columbia River Estuary Study Taskforce \\
\hline CRFMP & Columbia River Fish Mitigation Project \\
\hline CWA & Clean Water Act \\
\hline CWPPRA & Coastal Wetlands Planning, Protection and Restoration Act \\
\hline ENSO & El Nino Southern Oscillation \\
\hline EPA & U.S. Environmental Protection Agency \\
\hline ESA & Endangered Species Act \\
\hline ESU & evolutionarily significant unit \\
\hline ETM & estuarine turbidity maxima \\
\hline FCRPS & Federal Columbia River Power System \\
\hline GI & general investigation \\
\hline GIS & geographic information system \\
\hline GLO & General Land Office \\
\hline HSM & Habitat Suitability Model \\
\hline IBI & Index of Biological Integrity \\
\hline ISAB & Independent Scientific Advisory Board \\
\hline ISRP & Independent Scientific Review Panel \\
\hline LCFRB & Lower Columbia Fish Recovery Board \\
\hline LCREP & Lower Columbia River Estuary Partnership \\
\hline MHHW & mean high high water \\
\hline MLLW & mean low low water \\
\hline NMFS & National Marine Fisheries Service (now called NOAA Fisheries) \\
\hline NOAA & National Oceanic and Atmospheric Administration \\
\hline NPCC & Northwest Power and Conservation Council (ex- Northwest Power Planning Council) \\
\hline
\end{tabular}


NRC National Research Council

NST Nearshore Science Team

OWEB Oregon Watershed Enhancement Board

PDO Pacific Decadal Oscillation

PNNL Pacific Northwest National Laboratory

RM river mile

RPA Reasonable and Prudent Alternative

SARE Salmon at River's End

SFBJV San Francisco Bay Joint Venture

SRFB Salmon Recovery Funding Board

SWG Science Work Group

TAT Technical Advisory Team

TRT Technical Recovery Team

USFWS U.S. Fish and Wildlife Service

WRDA Water Resources Development Act

WRIA Watershed Resource Inventory Area 


\section{Glossary}

Adaptive Management - A process for testing hypotheses through management experiments in natural systems, collecting and interpreting new information, and making changes based on monitoring information to improve the management of ecosystems; i.e., "learning by doing."

Conceptual Model - A graphical representation or a simple set of diagrams that illustrate a set of relationships among factors important to the function of an ecosystem or its subsystems.

Conservation - Defined in Table 2.3.

Creation - Defined in Table 2.3.

Disturbance - Any relatively discrete event in time that disrupts or alters some portion or portions of an ecosystem.

Ecosystem - A community of organisms in a given area together with their physical environment and its characteristic climate.

Ecosystem Attribute - A component of a natural system or conceptual model of a system. A subset of the potential biological elements or components of a natural system.

Ecosystem Function - Ecosystem function is defined as the role the plant and animal species play in the ecosystem, including primary production, prey production, refuge, water storage, nutrient cycling, etc.

Ecosystem Process - Ecosystem processes are any interaction among physicochemical and biological elements of an ecosystem that involve changes in character or state.

Ecosystem Structure - Ecosystem structure is defined as the types, distribution, abundances, and physical attributes of the plant and animal species comprising the ecosystem.

Effectiveness Monitoring - Activities designed and undertaken to assess how well a particular restoration project performs.

Enhancement - Defined in Table 2.3.

Estuarine Subsystem - For the purpose of this document, defined as the part of the CRE between the river mouth and river mile 46.

Estuarine Turbidity Maxima - Circulation phenomena in an estuary that traps particles and promotes biogeochemical, microbial and ecological processes that sustain a dominant pathway in the estuary's food web (from http://depts.washington.edu/cretmweb/).

Estuary - The tidally influenced waters of a river. In the Columbia River, the estuary is the portion from the mouth (RM 0) to Bonneville Dam (RM 146). 
Habitat - The physical, biological, and chemical characteristics of a specific unit of the environment occupied by a specific plant or animal.

Intervention - To act for the purpose of making modifications.

Lower Columbia River - The tidally-influenced freshwater part of the estuary from RM 46 to RM 146.

Ocean-type - Life history pattern for salmon in which juveniles migrate to sea as subyearlings.

Oligohaline - Water having low salinity.

Plume - The layer of Columbia River water in the nearshore Pacific Ocean.

Project Sponsors - Entities advocating a particular habitat restoration project.

Protection - Defined in Table 2.3.

Restoration - Defined in Table 2.3.

Restricted Development - A land use policy that limits or prohibits potentially harmful land use activities.

Stream-type - Life history pattern for salmon in which juveniles migrate to sea as yearlings.

Stressor - A component of a conceptual model. A physical, chemical, or biological entity or process that induces effects on individuals, populations, communities, or ecosystems.

Subarea - A portion of a larger area that has unique characteristics.

Subsystem - A system within the larger ecosystem that has unique characteristics.

Tidal Freshwater Subsystem - For the purpose of this document, defined as the part of the CRE between RM 46 and RM 146 (Bonneville Dam). 


\subsection{Introduction}

\subsection{Background}

The Columbia River estuary (CRE) extends from the mouth to Bonneville Dam (Figure 1.1) and is part of the migration pathway for anadromous fish in the Columbia Basin, including salmon listed under the Endangered Species Act (ESA). Salmon in various stages of life, from fry to adults, use tidal wetlands in the Columbia River estuary to feed (McCabe et al. 1986), find refuge from predators (McCabe et al. 1983), and transition physiologically from freshwater to saltwater (Bottom et al. 2001). Over the last 100 years, however, the area of some types of wetland habitat in this region has decreased by as much as $70 \%$ from historical levels because of dike and levee building, development, and other activities (Lower Columbia River Estuary Partnership 1999). In addition, Federal Columbia River Power System (FCRPS) operations have altered river flow patterns and, hence, sediment transport and salinity intrusion (Weitkamp 1993). Unprecedented efforts are underway in the tributaries and at the mainstem dams of the Columbia Basin to rebuild migratory salmonid populations. More recently, the importance of the CRE has been recognized (Bisson et al. 2000; National Marine Fisheries Service 2000; Northwest Power Planning Council 2000) and ecological restoration ${ }^{1}$ activities have begun in earnest.

In their 2000 Biological Opinion (BiOp) on operation of the FCRPS, the National Marine Fisheries Service (NMFS) recognized the importance of the Columbia River estuary (CRE) to salmonid survival. Reasonable Prudent Alternative (RPA) Action 159 states, "BPA and the Corps, working with LCREP and NMFS, shall develop a plan addressing the habitat needs of salmon and steelhead in the Estuary" (NMFS 2000). This document addresses RPA Action 159. It uses the best available science and builds on ongoing restoration planning, salmonid recovery planning, and on-the-ground restoration efforts. The document will help guide habitat restoration in the CRE funded by the Action Agencies (BPA and COE) and others. It advocates an ecosystem-based approach to restoration because ecosystem processes are necessary to develop and maintain habitat types and functions important to the support of salmonids and other native species (National Research Council [NRC] 1992). Therefore, this document provides a broad restoration perspective that other interested parties, besides the BPA and the COE, are anticipated to find useful.

\footnotetext{
${ }^{1}$ In this document, the term "restoration" generally refers to any or all of the five fundamental restoration approaches commonly reported in the literature: creation, enhancement, restoration, conservation, and protection. These approaches are defined in Section 2.1.3. When used to refer specifically to restoration as a particular strategy, we will italicize the word; otherwise, assume usage is in the general sense.
} 


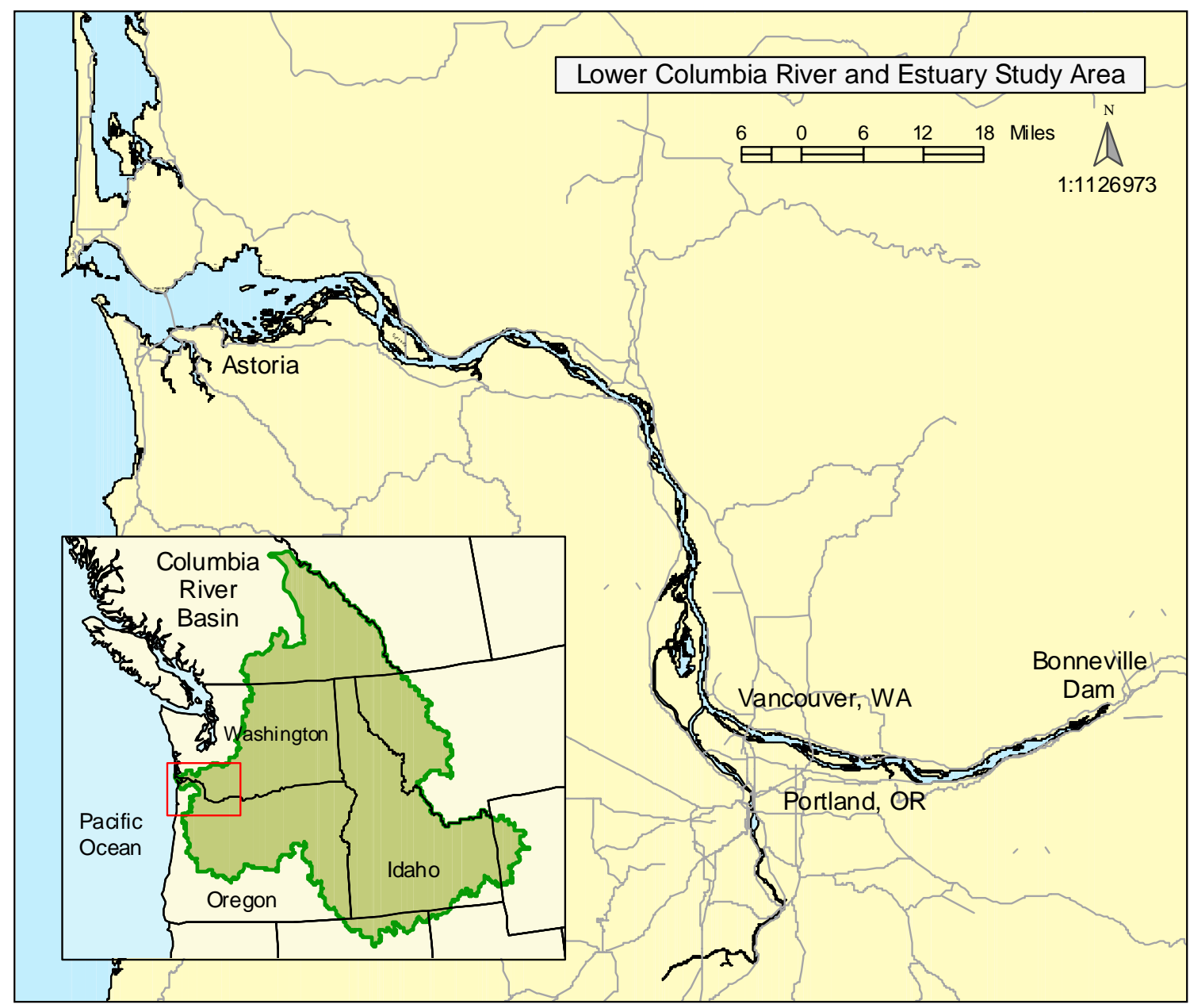

Figure 1.1. Columbia River Estuary Study Area from the Mouth to Bonneville Dam.

The BPA and COE have an overall goal to protect and restore 10,000 acres of wetlands in the CRE, as mandated by RPA Action 160 in the BiOp. This goal is consistent with Action 2 in the Estuary Partnership's Comprehensive Conservation and Management Plan (LCREP 1999). One purpose of this document is to provide a scientific basis and implementation guidelines for projects directed at helping to attain this restoration goal.

The document incorporates the habitat restoration planning efforts over the last few years by the Columbia River Estuary Study Taskforce (CREST), the Lower Columbia River Estuary Partnership (Estuary Partnership), and the Estuary Partnership’s Science Work Group (SWG). These collaborators developed a systematic, ecosystem-based approach to select and implement habitat conservation and restoration projects in the Columbia River estuary. Previous habitat restoration efforts were primarily opportunistic in nature. This approach was not strategically focused on the overall ecosystem, nor did it directly consider salmonid habitat needs. Several key factors and uncertainties have prevented, until recently, development of a formal restoration approach for the CRE. These factors include 1) limited understanding of lower river and estuary ecosystem functions and structures, particularly relative to salmonids and FCRPS effects; 2) incomplete data on quantity and quality of existing habitat; 3) lack of accepted project selection criteria; 4) no coordinating framework, management structure, or mutually agreed upon goals to bring stakeholders together in a meaningful fashion; 5) no agreement on how to 
monitor habitat, and 6) no baseline habitat data, making it difficult to characterize temporal changes and evaluate restoration project success. In the past few years, however, work was initiated and is ongoing today in the CRE to research juvenile salmon usage of various habitats, map and quantify existing habitats, establish project selection criteria, develop a management structure, and identify monitoring and adaptive management protocols. This habitat restoration approach combines these efforts into a unified framework.

The lack of a coordinated management structure to efficiently implement meaningful restoration projects is a particularly important issue. Coordination needs to occur among federal, state, and local agencies, tribes, non-governmental organizations, and other interested parties. The Estuary Partnership has made substantial progress in this regard, but their efforts need to be enhanced as the resource base for CRE restoration increases. With many of the new funds coming from the federal government, deeper coordination between the federal and non-federal agencies will be especially important. In this document, improved coordination and collaboration among the stakeholders is highly recommended to ensure the implementation of scientifically sound restoration projects with sufficient monitoring, data management, and evaluation.

The foundation to initiate a comprehensive and collaborative restoration effort is embodied in this document. The overall approach entails a broad-based, organized effort to identify and implement actions beneficial for CRE ecosystems and, hence, for the salmon and steelhead using this migration pathway. The strategy embraces an ecosystem-based, "top-down" approach to provide overall structure and an ecosystem perspective in conjunction with a "bottom-up" approach to garner local support for individual projects. However, the document does not include proposals for specific restoration projects, nor does it advocate particular projects. This approach may be used to inform restoration efforts outside the purview of the Action Agencies. The approach contained herein provides strategies and guidelines designed to take advantage of the growing momentum for restoration in the CRE.

\subsection{Program Goal and Principles}

The CRE habitat restoration program's goal and principles address habitat restoration projects using an ecosystem context. Since restoration of an entire ecosystem is not generally practical, individual habitat restoration projects have the greatest likelihood of success when they are implemented with an ecosystem perspective, i.e., they are ecosystem-based. The program goal and underlying principles are as follows:

Goal: Implementation of well-coordinated, scientifically sound projects designed to enhance, protect, conserve, restore, and create 10,000 acres of tidal wetlands and other key habitats to aid rebuilding of ESA-listed salmon populations and native species using the Columbia River estuary. 


\section{Principles:}

Ecological Basis -- Projects are founded on the best available ecological restoration science, implemented in an ecosystem context, and developed with the intent to restore relevant ecological processes.

Adaptive Management -- Projects incorporate adaptive management practices with testable hypotheses to track ecological responses to a given restoration effort.

Coordination and Communication -- Projects are implemented in a coordinated, open process and scientific results from monitoring and evaluation are communicated widely and readily accessible.

\subsection{Study Objectives and Tasks}

The intent of this document is to provide a scientific basis and implementation guidelines for a habitat restoration program designed to improve ecosystem functions and enhance juvenile salmonid survival in the Columbia River estuary (CRE). Embodied in Objective 1 below, the document links output from a conceptual model, a description of juvenile salmon usage, and an assessment of existing habitat conditions with fundamental strategies for restoration to drive a prioritization process for the CRE. The document draws from the fields of restoration ecology, landscape ecology, and conservation biology to offer the most useful restoration strategies for the CRE. In Objective 2 below, the focus is on guidelines to implement habitat restoration at the project and program levels. The third and final objective addresses next steps arising from this approach. Specifically, the study objectives and associated tasks are as follows:

Objective 1: Establish a scientific basis for ecological restoration of juvenile salmon habitat in the Columbia River estuary.

Task 1: Explain the fundamentals of ecosystem-based habitat restoration.

Task 2: Provide a conceptual ecosystem model of the CRE.

Task 3: Summarize available data on habitat and ecosystem requirements for juvenile salmon in the CRE and list critical uncertainties in this knowledge base.

Task 4: Describe current conditions and ecological changes over the last century in the CRE.

Task 5: Identify and prioritize habitat restoration strategies for subareas of the CRE.

Objective 2: Develop implementation guidelines for CRE restoration projects in the context of and consistent with ongoing efforts.

Task 1: Describe types of restoration projects for the CRE consistent with the recommended strategies.

Task 2: Refine project selection guidelines.

Task 3: Describe a process to implement habitat restoration projects, including phases for planning, funding, constructing, monitoring and evaluating, and adaptively managing CRE restoration efforts. 
Objective 3: Discuss constraints to implementation and recommend next steps to fulfill the goal of the CRE habitat restoration program.

\subsection{Study Area}

The Columbia River estuary study area is at the terminus of the Columbia Basin watershed (Figures 1.1 and 1.2). The Columbia River is the second largest river in the United States. Its watershed comprises approximately $650,000 \mathrm{~km}^{2}$, has a mean annual discharge of 5,500 $\mathrm{m}^{3} \mathrm{~s}^{-1}$, and crosses two major continental mountain ranges (Bottom et al. 2001; Neal 1972). Because of its latitudinal position, it is affected by the climatic cycles of the Pacific Decadal Oscillation (PDO) and El Nino-Southern Oscillation (ENSO). Historically, the Columbia River was a highly dynamic system that was shaped by a variety of natural physical forces including volcanic activity, floods, and climate changes throughout time. Morgan et al. (1979) provide an annotated bibliography of the CRE.

The study area for this document includes two major physiographic subsystems of the CRE, the estuarine and tidal freshwater subsystems:

1. Estuarine Subsystem: Columbia River mouth to Puget Island (River Mile [RM] 0-46). Includes seven subareas - Entrance, Mixing Zone, Youngs Bay, Baker Bay, Grays Bay, Cathlamet Bay, and Upper Estuary.

2. Tidal Freshwater Subsystem: Puget Island to Bonneville Dam (river mile 46-146). Includes two subareas - RM 46-105 and RM 105-146.

These subsystems are highly dynamic, resulting in part from interactions between seasonal flow and salinity-tidal regimes. Designation of these subsystems was based on the estuary sub-areas developed during the Columbia River Estuary Data Development Program (Simenstad et al. 1984). Each subsystem and its respective subarea(s) have unique characteristics, e.g., habitat structure, salinity concentrations, and sediment composition. Historical and current conditions in the study area are described in more detail in Section 2.3.

The geographic extent of the CRE is the tidally influenced region of the Columbia River from its mouth to Bonneville Dam 146 miles RM upstream (Bottom et al. 2001). The study area does not include the Willamette River or other tributaries in the lower 146 miles of the Columbia River. Although RPA Action 160 calls for restoration activities from the mouth to RM 46 (NMFS 2000), we defined the study area for the document called for in Action 159 as the tidally influenced region from RM 0 to RM 146 because the tidal freshwater reach in RM 46-146 connects mainstem reservoir and estuarine habitats that are important to juvenile salmonids. However, because the restoration approach contained herein concentrates on actions that would restore components of the estuarine and tidal portion of the landscape that are utilized by salmon, as such its does not extend into non-tidal areas where salmon spawning and early life history rearing occurs. Also, it does not deal with plume, coastal, or oceanic habitats. Hence, this document must not be viewed as a comprehensive restoration approach for all salmon habitats. 


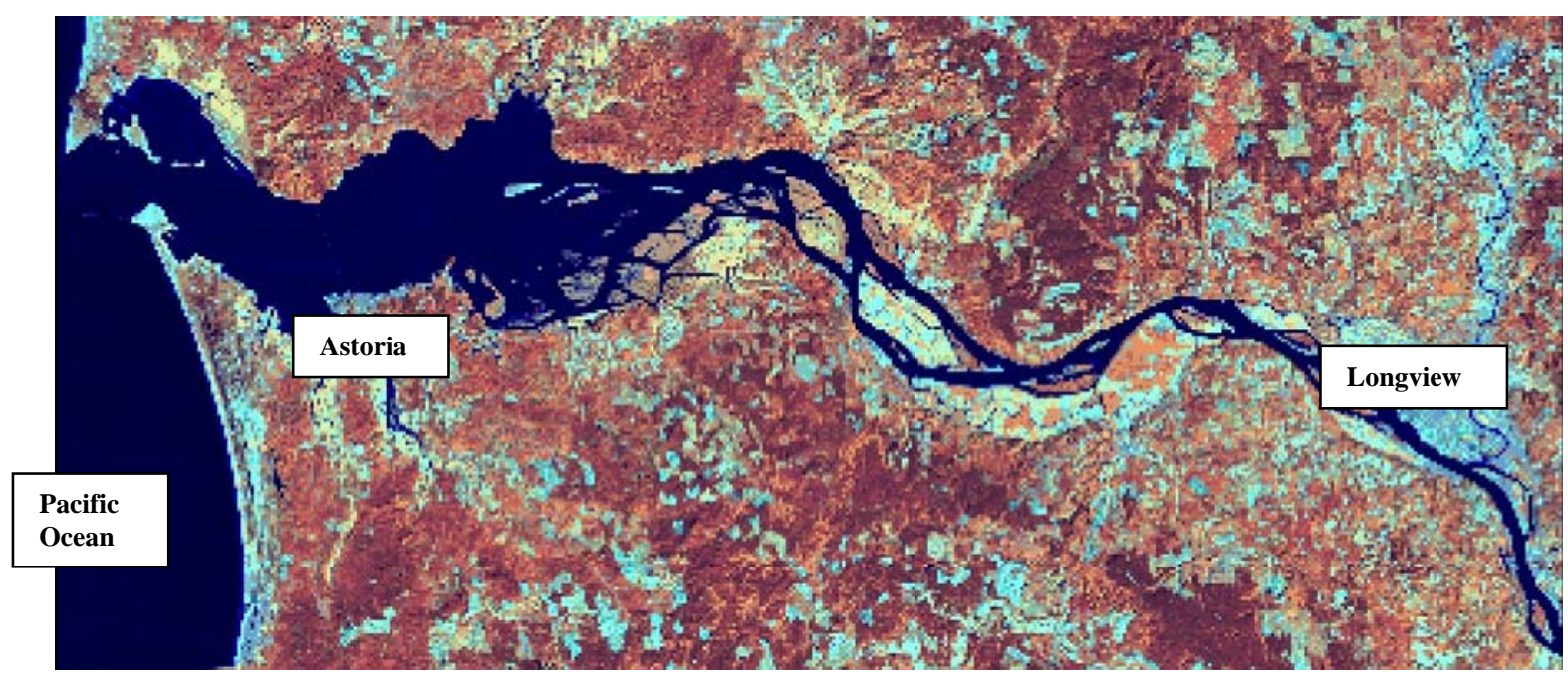

Figure 1.2. Satellite Photograph of the Lower Portion of the Columbia River Estuary (from Garano et al. 2002)

\subsection{Related Restoration Initiatives}

This habitat restoration approach for the CRE incorporates key features of other local and regional initiatives. These initiatives are explained briefly in this section (for more detail, see Appendix A). Locally, there are numerous efforts in the CRE to restore aquatic habitats. The approach contained herein will interface and be coordinated with the ongoing and proposed locally originated projects. Regionally, essential collaboration among many agencies of federal, state, and tribal governments involved in habitat restoration in the CRE is occurring through the BPA, the COE, the Estuary Partnership, the Lower Columbia Fish Recovery Board, and the NPCC (see Appendix A). Of particular note are the relevant efforts in the CRE to implement the NOAA Fisheries BiOp and the Northwest Power and Conservation Council Fish and Wildlife Program.

For the BiOp, the habitat restoration approach responding the RPA Action 159 is related to other estuary RPA actions. Action 158 will provide an overall programmatic action plan for the RPA implementation in the estuary. Action 160 calls for implementation of on-the-ground habitat protection and enhancement work. Action 161 mandates a research, monitoring and evaluation (RME) program. Monitoring of restoration projects will be an element of the RME plan for the CRE being developed for Action 161. In addition under Action 161, research in the estuary involving survival, habitat usage, and linkages between physical conditions and biological responses will help guide restoration planning. Actions 162 and 194 entail conceptual ecosystem and physical modeling efforts, respectively. Action 195 addresses sources of mortality to listed-salmonid smolts below Bonneville Dam. Actions 196 and 197 involve study of salmonid usage in the estuary and plume. Finally, the NOAA Fisheries Technical Recovery Team (TRT) for the Willamette/Lower Columbia, including the CRE, is developing a recovery plan for listed salmonid fishes in that region (see Appendix A, Section A.1, for more details).

The NPCC's Fish and Wildlife Program involves a subbasin planning process to organize, coordinate, and implement projects to rebuild depressed salmon stocks. To initiate the process for the CRE, the Estuary Partnership developed a subbasin summary (Marriott et al. 2001). It is intended that 
subbasin planners incorporate the habitat restoration approach herein as they develop the CRE subbasin plan scheduled for release in May 2004.

Nationally, the approach is consistent with the National Strategy to Restore Coastal and Estuarine Habitat (Restore America's Estuaries and NOAA 2003) and the imperative for estuarine habitat restoration embodied in the Estuary Restoration Act of 2000. For example, the Strategy states, "Implement restoration projects to provide healthy ecosystems that support wildlife, fish, and shellfish..."

There are five major coastal and estuarine restoration programs either in the planning stage or being implemented in the United States (Table 1.1). These are the Mississippi Delta, the San Francisco BayDelta, the Florida Everglades, the Chesapeake Bay, and the Puget Sound Nearshore. Compared to these programs, the spatial extent of the CRE habitat restoration program is roughly the same order of magnitude, but the expenditure level at this time is one to three orders of magnitude higher for the others than the CRE. The approach outlined in the present report has gleaned information on scope and approach from these other major programs. Large restoration programs provide a number of lessons that are applicable to the present document, such as:

- A coordinating body for the program is essential.

- A goal statement for the program is essential and will drive what actions are carried out and to some extent where these actions occur.

- Development of a conceptual model helps organize the understanding of the system and better define what is known and unknown, and what action might have the most direct benefit in reaching a goal.

- Directed research provides essential information that can help refine the model and help target actions.

- Projects can proceed in the face of some uncertainties, as long as monitoring is conducted to learn from the project.

- Site assessment can reduce uncertainties, which can lead to better results and less costly projects.

- Failed projects can provide useful information.

- A coordinated monitoring program is essential.

- Regularly scheduled assessments of the data are essential.

- Dissemination of the information to technical audiences and the public is essential for long-term viability of the program. 
Table 1.1. Brief Summaries of Major Regional Habitat Restoration Programs in the United States.

\section{Mississippi Delta Marsh Restoration}

On the order of $80 \%$ of the loss of coastal wetlands in the United States occurs on the Mississippi River delta in the state of Louisiana. Presently, because of a variety of factors, wetlands are lost at a rate of $65-90 \mathrm{~km}^{2} \mathrm{y}^{-}$

1 . Because of the low-lying topography, wetland loss also threatens roads, pipelines, and the general infrastructure of the region. The Coastal Wetlands Planning, Protection and Restoration Act (CWPPRA) (Public Law 101-646) (http://www.nmfs.noaa.gov/habitat/restoration/projects-programs/CWPPRA/) in 1990 was passed to address this problem. The primary strategy to restoring wetlands is to divert freshwater containing nutrients and sediments into wetland areas adjacent to the Mississippi River. In addition, shoreline protection measures are being implemented to protect vulnerable leading edges of the marsh. Between 1994 and 2001, 60 projects have been constructed covering approximately 600,000 acres. The program is expensive, and some of the strategies used for restoration are unproven. Hence, early on, planners incorporated a strong monitoring program into an adaptive management framework for the program (Raynie and Visser 2002). The primary objective of the adaptive management program is to close the feedback loops between different phases of project planning, implementation, and results monitoring. The program is designed to learn from the implemented projects and design more successful future projects. Toward this end, interdisciplinary teams meet regularly (i.e., every six months) to review data and develop recommendations for changes as needed. This team involves member working on CWPPRA projects as well as other programs. For example, the COE has several restoration projects on the delta. By working together, the members benefit from the learning process and can use the information to improve their own projects.

\section{San Francisco Bay-Delta Restoration Program}

This program is commonly referred to as the CALFED Bay-Delta Restoration Program because it involves a consortium of 11 state and 13 federal agencies with the mission of developing and implementing a longterm comprehensive plan to restore ecological health and improve water management for beneficial uses of the Bay-delta system (http://calwater.ca.gov/). The program was established in 1994 under a Framework Agreement for management and regulatory responsibility in the system. The program has three phases: 1) define problems and develop a range of solution alternatives; 2) prepare environmental documents to satisfy state and federal laws and regulations; and, 3) implement preferred alternative. As of 2003, the program had received \$398,000,000 in state, federal, and stakeholder funds and cost-sharing agreements, which have been awarded to 379 habitat restoration projects. The program has a strong science base to support phase 1 . This science program, which is developed through a peer reviewed proposal process, is established to integrate world-class science and peer review into every aspect of the Bay-Delta Program to guide decisions and evaluate actions that are critical to program success. A total of 23 "science issues" have been identified ranging from chemical contamination (arsenic and mercury) to watershed-wide sediment budget assessment. These issues represent key stressors to the system. A review of the program published in 2003 (Environment. January- February, vol. 45, p. 30) noted a number of points where the program was encountering difficulties including securing adequate and consistent funding, lack of authority by CALFED to force agencies to cooperate, and contracting problems resulting in project delays. On the positive side, the report noted that the CALFED program was facilitating open discussions between stakeholders and that the relationships built through the program have changed public policies.

\section{Florida Everglades Restoration Program}

The Central and South Florida Project, authorized by Congress in the late 1940s, was implemented to ensure adequate water supply for south Florida and its natural systems, as well as flood protection for urban and agriculture centers. This massive project constructed canals and diversion channels and water control structures to achieve these goals. By the late 1980s it was evident that this level of hydrological manipulation had resulted in a long list of environmental ills including 50\% reduction in Everglades marshes, die-offs of tens of thousands of acres of sea grasses in Florida Bay, loss of over half of the islands in the marshes, and listing of over 60 species as threatened or endangered (Ogden et al. 2003). The Comprehensive Everglades Restoration Plan (CERP) authorized by Congress in 1992 was designed to rectify these ills in what is commonly referred to as the largest 
restoration project in the world. It was clear that because of the massive scale of changes in the system, full restoration was not possible. The plan is to shift the system from its present state to somewhere along the spectrum from current condition to pre-drainage condition. The restoration planning process is strongly science-based, and has utilized field and modeling studies to develop a restoration plan for the system. The science team, which directs the research and monitoring and will evaluate the results, developed a set of conceptual models to help identify key uncertainties. These uncertainties were subject to additional study and will be evaluated further during monitoring. A review has been done of existing monitoring data and how these data might be applied to assess performance of the projects. The science team has developed a comprehensive monitoring and assessment program and the implementation phase has been started.

\section{Chesapeake Bay Program}

The Chesapeake Bay Program is a multi-jurisdictional partnership that is working successfully to restore and protect the Bay and its resources. Since the Bay Program's inception, the federal and state partners have met many goals and worked toward others in the effort to restore the Bay. The partners work to restore the Bay by: sound land use, restoring water quality and habitat, monitoring, managing fisheries, and computer modeling. This work will help return the Chesapeake Bay to a condition that more closely resembles its original productivity, with underwater grasses and oyster reefs lining the Bay's shallow edges. More information can be found at: http://www.chesapeakebay.net/restrtn.htm.

\section{Puget Sound Nearshore Ecosystem Restoration Program}

Puget Sound is one of the nation's largest and most complex inland seas and provides habitat for a wide variety of marine resources. Because of development and contaminant discharge, the nearshore (beaches) habitats of Puget Sound have experienced significant changes, which have resulted in declines in many of these species, including highly prized species such as salmon. A general investigation conducted by the U.S. Army Corps of Engineers in 2000 identified a direct link between healthy nearshore habitat and the physical shoreline condition. Based on this study, the COE is in the process of planning a major restoration program for the nearshore of Puget Sound. To date, a science team has developed a conceptual model of the nearshore to help direct key research and to assist in understanding what needs to be restored. In 2003, the science team produced a document on guidance for protection and restoration of the nearshore ecosystem of Puget Sound (Fresh et al. 2003). The program is expected to ramp up in scope and budget over the next several years, with full implementation of restoration actions beginning in FY2008.

\subsection{Document Organization}

The document is organized by study objectives and tasks (see Section 1.3). Following the introduction, the scientific basis for restoration is presented in Section 2. The scientific basis starts with the fundamentals of wetland habitat restoration, includes a conceptual model, a description of juvenile salmonid usage of the CRE, and a habitat change analysis, and ends with recommended restoration strategies for the CRE. In Section 3, guidelines for habitat restoration implementation are described. These include types of projects, project selection criteria, and detailed explanation of restoration project phases. In Section 4, constraints to implementation are discussed and next steps are recommended. Section 4 addresses the issue of how to use the approach. The literature cited is in Section 5. Appendix A details other related habitat restoration efforts and programs in the CRE. Appendix B contains a conceptual ecosystem model for the CRE. 


\subsection{Scientific Basis}

The purpose of this section is to develop the scientific basis for ecological restoration of salmon habitat in the CRE and to arrive at recommended restoration strategies. The scientific basis draws from past experiences in the CRE and other systems and from the growing body of literature on estuarine restoration and salmonid ecology. The framework for restoration used here emphasizes the reestablishment of natural processes needed to build and maintain habitat structure and function. The overarching premise for restoration of the CRE is that re-establishing the factors that control the development, dynamics, and maintenance of native habitat structure will result in restoration of natural habitat and ecosystem functions and processes. Among the functions restored would be the support of habitats utilized by salmonids. This ecosystem/process-based approach encompasses both salmonid restoration and the attendant ecosystem restoration issues. The scientific basis includes fundamentals of ecological restoration, a conceptual CRE ecosystem model, habitat requirements for juvenile salmonids, and habitat changes in the CRE, and recommended restoration strategies for the CRE.

\subsection{Fundamentals of Ecological Restoration}

This section provides a primer on ecological restoration. It includes a general model for ecosystem state, the role of landscape ecology, generic restoration strategies, an explanation of sustainable development, and the influence of disturbance. These fundamentals are applied to the CRE later in the report.

\subsubsection{General Model for Ecosystem State}

The general goal for restoration is to move the ecosystem from a less desirable condition to a more desirable condition as quickly as possible. A general model for ecosystem state (Figure 2.1) is a way to visualize the present (disturbed) and historical (undisturbed) "states" of the system, as well as to identify restoration goals (Thom 1997). First, it is assumed that there is a positive relationship between the structure 1 of an ecosystem and the function 2 of that ecosystem. Next, the system condition on both axes is divided into low, moderate, and high or optimal. The reasons for dividing the figure into three levels is to acknowledge two major sources of uncertainty: 1) our inability to accurately quantify the relationship between structural and functional ecosystem components; and 2) our inability to accurately predict the dynamic nature of regular periodic and stochastic natural variability associated with structural conditions and functional conditions (Shreffler and Thom 1993; Hobbs and Norton 1996). The three levels along each axis are qualitative indicator variables (e.g., acreage) related to the structural condition (e.g., the size of the pond-wetland interface) and the functional conditions (e.g., the number of ducks nesting at this interface). This means that we believe that an ecosystem under optimal conditions of structure and function can have values that vary over a certain range because of natural dynamics, that

\footnotetext{
${ }^{1}$ Ecosystem structure is defined as the types, distribution, abundances, and physical attributes of the plant and animal species comprising the ecosystem.

${ }^{2}$ Ecosystem function is defined as the role the plant and animal species play in the ecosystem,. Including primary production, prey production, refuge, water storage, nutrient cycling, etc.
} 
this range is the target we are aiming the restoration project at, and if we hit within this range we can consider the project a success.

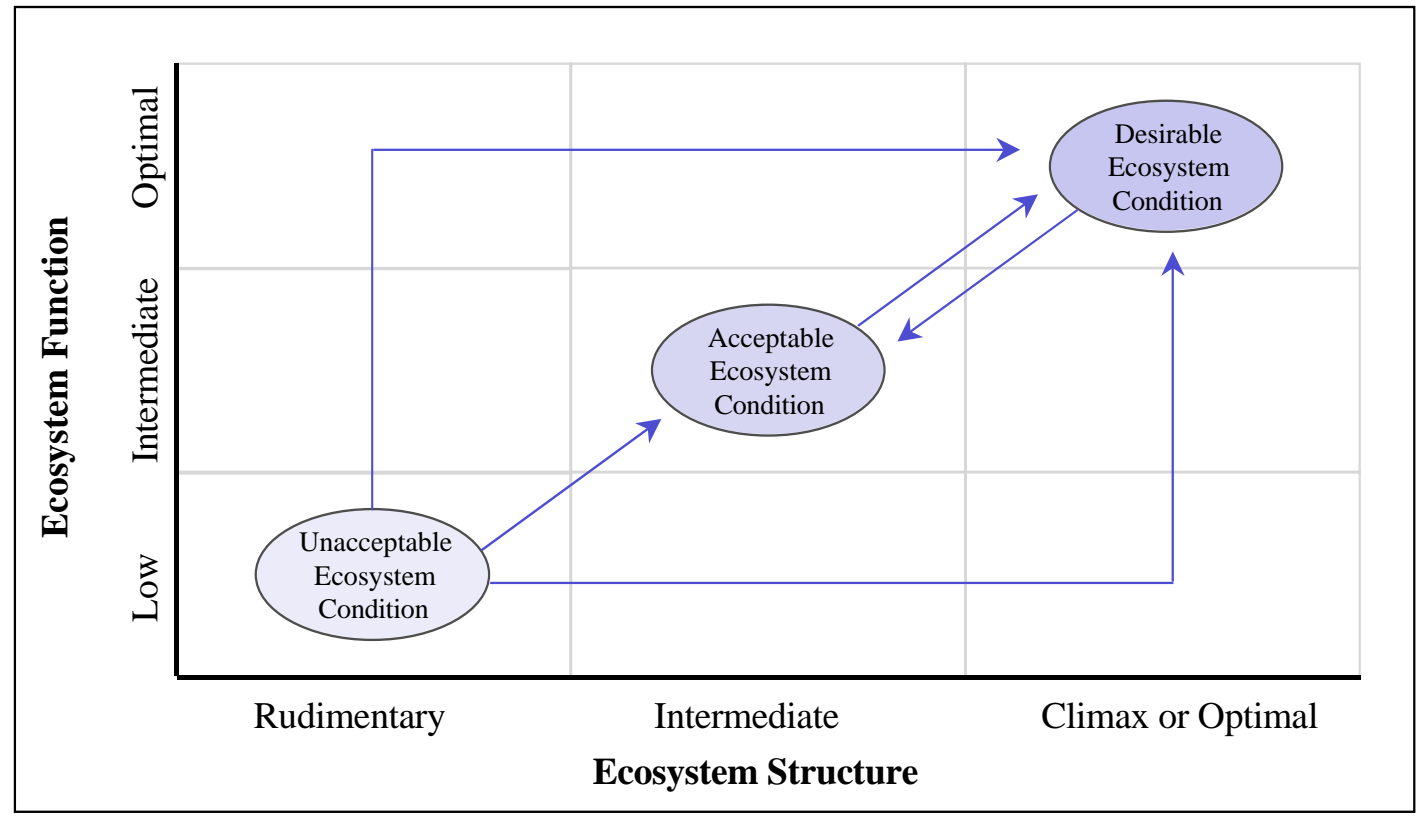

Figure 2.1. General Model of Ecosystem State. An ecosystem or habitat that is in rudimentary condition with low functioning develops into a system with optimal structure and functioning (after Thom 2000). Development can take several pathways and can oscillate between system states.

The natural climax structure of an ecosystem, habitat, or community has a corresponding and predictable functional condition. The upper right hand box, then, would represent a system that was fully developed, optimal biodiversity, self-maintaining, and resilient to disturbances. The upper right hand box labeled "Desired Ecosystem State" might represent the predisturbance conditions of the CRE. The upper right hand box can also represent the desired state of the system following restoration. The present condition of the CRE system is altered from prehistorical conditions, and it is not in an entirely desirable state. The structure and functioning of the system is different than prior to hydrological modification and other anthropomorphic or natural changes, and it probably has reached an equilibrium in this altered state. The growing body of information indicates that the survival of salmon may be dependent on return of the estuary to a less altered state (e.g., Bottom et al. 2001; Karieva et al. 2000). With regard to salmon and the habitats that support salmon, parts of the CRE now exist in what can be termed an Unacceptable Ecosystem Condition.

Table 2.1 provides an example list of structural and functional attributes that are relevant to the CRE. These two sets of attributes are at least correlated if not proven to be functionally linked. For example, as wetland size and complexity (i.e., number of species) increases, so does animal diversity (Forman and Godron 1986). Furthermore, the ability of a wetland to trap and retain sediments is a function of its size. In general, the stronger the proven linkage, the better it is for use in planning restoration actions.

Finally, this development model also indicates that if the system is not in the Desirable state, then there are potential explanations and options for actions that can be developed from the conceptual model (Thom 1997; 2000). For example, very poor ocean conditions could negate any incremental increase in juvenile salmon survival resulting from estuary restoration projects. Hence, if enhanced salmon returns 
are a functional metric, assessment of both factors within the estuary and outside the estuary must be brought to bear in assessing the reasons for the poor performance of the restoration effort.

Table 2.1. Sample list of selected structural and related functional attributes for estuarine systems.

\begin{tabular}{ll}
\hline Structural Attribute & Functional Attribute \\
\hline Area of tidal swamp & Tidal swamp net aerial primary productivity \\
Area of tidal marsh & Tidal marsh sediment trapping capacity \\
Plant species richness & Marsh animal species richness \\
Plant species abundances & Marsh animal species abundance \\
Plant canopy height & Marsh bird nesting \\
Length of marsh-channel edge & Juvenile salmonid prey production \\
Marsh patch size & Animal species diversity \\
Ratio of marsh edge length to interior area & Juvenile salmon residence time \\
\hline
\end{tabular}

\subsubsection{Role of Landscape Ecology}

Restoration projects are now utilizing the concepts and principals of landscape ecology to improve the functioning and success of projects (Kentula 1997). Landscape ecology deals with the effect of the spatial extent, heterogeneity, and geometry of elements (e.g., habitats) of the landscape on the flow of energy, animals, and materials through the landscape. One of the fundamental lessons of landscape ecology is that most elements within a landscape (e.g., watershed) function best when integrated with all other elements of the landscape. The field represents an integrated approach to understanding how large systems function (Forman and Godron 1986). Principles are emerging from the field that have direct applicability to planning and design of estuarine restoration. Human activities are fragmenting natural landscapes into fewer and fewer small pieces at an increasing rate (Weins 1985). The effect of fragmentation and habitat shrinkage is less exchange of materials and species among habitats. Increasing fragmentation and decreasing habitat size has been shown to be responsible for local extinction of some populations (Gonzales et al. 1998; Earn et al. 2000). Minimum area, shape, corridors, and buffers are useful landscape ecology concepts that can be applied to habitat restoration projects (Table 2.2).

The principles of landscape ecology present a framework for reducing fragmentation and designing restoration to limit local extinction. These principles revolve around the facts that a landscape is a heterogeneous matrix of smaller elements, and that the arrangement, size, productivity, resilience to disturbance, etc. of these elements within the matrix will affect the flow of energy, animals, and materials through the landscape. Removal or degradation of one or more elements may lead to the dysfunctional performance of the remaining elements. In deciding on restoration strategies and sites, for example, it is useful to identify and consider the dysfunctional or absent elements. 
Table 2.2. Explanations of Certain Landscape Ecology Concepts.

\begin{tabular}{ll}
\hline Concept & Explanation \\
\hline Minimum Area & $\begin{array}{l}\text { The minimum area or size of a project required for an estuarine habitat to become fully } \\
\text { functional is difficult to estimate because of the lack of empirical data from the CRE } \\
\text { and the Pacific Northwest in general. Size estimates are a function of the minimum area } \\
\text { needed to attract the species of interest, the size of the species, their behavior within the } \\
\text { habitat, and required buffers. In addition, the habitat(s) must be stable over time, and } \\
\text { with increased size comes stability. } \\
\text { The shape of a patch or contiguous habitat affects the types and number of species in } \\
\text { the patch. Species show preferences for edges or interiors of patches. In particular, } \\
\text { juvenile salmon are believed to forage heavily at the marsh-mudflat interface and at the } \\
\text { edge of habitat patches (Bottom et al. 2001). } \\
\text { A corridor is a narrow strip of habitat that differs from the habitats on either side. } \\
\text { Corridors form very important routes of migration for many species. Corridors } \\
\text { represent a more or less protected route of ingress and egress to habitats. Relative to } \\
\text { restoration planning, corridors between sources of recolonizing species and the restored } \\
\text { habitat are critical. If corridors are not present, the restoration effort has little chance of } \\
\text { success no matter how well it is constructed. Corridors may also function as habitat for } \\
\text { some species, and barriers or filters (e.g., riparian buffer zones) (Foman and Godron } \\
\text { 1986). } \\
\text { A vegetated buffer surrounding an aquatic habitat reduces disturbances from noises, } \\
\text { wind, contaminated runoff, and movement. Without a high quality buffer, the functions } \\
\text { and stability of the aquatic habitat may be compromised. }\end{array}$ \\
Buffers &
\end{tabular}

Of particular relevance to estuarine restoration are the concepts of habitat size, shape, and accessibility (Simenstad and Thom 1992; Shreffler and Thom 1993; Bottom et al. 2001; Simenstad and Cordell 2000). Knowledge of the behavioral patterns of target species or species groups can greatly help in maximizing the site selection and design process. The National Research Council (NRC 1991, page 347-348) report viewed landscape ecology as a method to design integrated aquatic ecosystem restoration projects. They concluded that, "Wherever possible...restoration of aquatic resources...should not be made on a small-scale, short-term, site-by-site basis, but should instead be made to promote the longterm sustainability of all aquatic resources in the landscape." Re-creating a self-maintaining ecosystem is more likely when the restoration effort is planned in the context of the landscape. The report, however, cautioned that the complexity of recreating landscapes should not be used as an excuse for postponing restoration efforts. Any shift of a damaged ecosystem to an improved ecological condition is preferable to allowing the system to remain damaged or to suffer further degradation. Recognizing that in some estuaries the extent of human disturbance and alteration may not provide opportunities for large-scale projects, incremental, opportunistic restoration projects can and should be undertaken under the larger umbrella of ecosystem restoration. 
Reiterating the earlier NRC (1992) report, the subsequent NRC (2001) report on wetland mitigation recommended that systems should be designed to incorporate natural processes and, to ensure long-term stability. Project sponsors should 1) consider the hydrogeomorphic and ecological landscape and climate and 2) adopt a dynamic landscape perspective. By this latter point, they meant that both the current and future conditions at the site and in the surrounding landscape should be considered when planning a project. This could mean preserving buffers surrounding the site and the connectivity to other wetlands.

Application to CRE: Most Pacific Northwest estuaries are part of a landscape that includes a steep, heavily wooded, watershed supplying ample flows. Oceanic input is also a major factor influencing processes in the estuary. The gradient of habitats generally encountered in an estuary includes wooded upland, riparian vegetation, swamps and marshes, tidal flats, sea grass meadows, and shallow to deep channels. This basic structure was formed through geomorphic processes that will have a controlling influence on any habitat that is constructed or restored. Furthermore, this natural system is a key to understanding the potential mix of habitat types (i.e., patches) that need to be incorporated into an estuarine landscape matrix.

\subsubsection{Generic Restoration Strategies}

The science of ecological restoration has defined strategies (Table 2.3) that provide guidance for restoration projects. Depending on site-specific characteristics and restoration project goals, more than one strategy may be appropriate for a site. In addition, multiple strategies may be employed at a site to maximize the benefit to the ecosystem. Later in the report, we describe how a strategy can be selected for a particular site. Ecologists also categorize restoration strategies as passive (conservation and protection) or active (creation, enhancement, restoration).

In Figure 2.2, we present six possible approaches to restoration. All try to exploit an aquatic ecosystem's capacity to self-adjust to change. Five approaches involve intervention. The sixth approach is based on the premise that through time, and with control of the sources of disturbance, it is possible for a degraded system to naturally recover. Intervention becomes necessary when an estuary is degraded to the point that it no longer has the capacity for self-maintenance and repair. The success of any of these four intervention approaches is dependent upon pollution abatement and efforts to restore good water quality. In turn, restoration of water quality is dependent on watershed conditions and any water quality restoration or protection activity must include remedial action in the watershed. Source control is a form of intervention that refers to abatement of sources of physical disturbances (e.g., flow regulation; disposal of dredged material) of the system. Without control of the sources of disturbance, the potential benefits of habitat restoration, enhancement, or creation can be compromised. These approaches are not mutually exclusive; within a particular estuary, several of these approaches may be integrated to achieve the overall restoration goal. Descriptions of six basic approaches follow. 
Table 2.3. Definitions of Restoration ${ }^{1}$ Strategies

\begin{tabular}{|c|c|c|}
\hline Strategy & Definition & Comments \\
\hline Conservation & $\begin{array}{l}\text { Maintenance of } \\
\text { biodiversity (Meffe et } \\
\text { al. 1994). }\end{array}$ & $\begin{array}{l}\text { Conservation biology is a synthetic field that applies the principles of } \\
\text { ecology, biogeography, population genetics, economics, sociology, } \\
\text { anthropology, philosophy and other theoretically based disciplines to } \\
\text { the maintenance of biological diversity. Conservation can allow } \\
\text { development to occur as long as biodiversity and the structure and } \\
\text { processes to maintain it are not affected. Restricted development is } \\
\text { an approach to conservation. }\end{array}$ \\
\hline Creation & $\begin{array}{l}\text { Bringing into being a } \\
\text { new ecosystem that } \\
\text { previously did not exist } \\
\text { on the site (NRC } \\
\text { 1992). }\end{array}$ & $\begin{array}{l}\text { In contrast to restoration, creation involves the conversion of one } \\
\text { habitat type or ecosystem into another. }\end{array}$ \\
\hline Enhancement & $\begin{array}{l}\text { Any improvement of a } \\
\text { structural or functional } \\
\text { ecosystem attribute } \\
\text { (NRC 1992). }\end{array}$ & $\begin{array}{l}\text { As noted by Lewis (1991), enhancement and restoration are often } \\
\text { confused. The intentional alteration of an existing habitat to provide } \\
\text { conditions that previously did not exist and which by consensus } \\
\text { increase one or more attributes is enhancement. Shreffler and Thom } \\
\text { (1993) found that, for estuarine systems, enhancement often meant } \\
\text { enhancement of selected attributes of the ecosystem such as } \\
\text { improving the quality or size of a tidal marsh or eelgrass meadow. }\end{array}$ \\
\hline Restoration & $\begin{array}{l}\text { Return of an ecosystem } \\
\text { to a close } \\
\text { approximation of its } \\
\text { previously existing } \\
\text { condition (e.g., Lewis } \\
\text { 1991, NRC 1992). }\end{array}$ & $\begin{array}{l}\text { Includes any form of restoration with the intent of improving habitat } \\
\text { to a state closely approximating a historical or pre-disturbance } \\
\text { condition. }\end{array}$ \\
\hline Protection & $\begin{array}{l}\text { Formal exclusion of } \\
\text { activities that may } \\
\text { negatively affect the } \\
\text { structure and/or } \\
\text { functioning of habitats } \\
\text { or ecosystems. }\end{array}$ & $\begin{array}{l}\text { Protection can also refer to protection of a species or group of species } \\
\text { through management actions such as elimination of harm to a species } \\
\text { directly or indirectly through damage of its habitat. Restricted } \\
\text { development and land use ordinances can also be used to exclude } \\
\text { unwanted activities as an approach to protection. }\end{array}$ \\
\hline \multicolumn{3}{|c|}{$\begin{array}{l}{ }^{1} \text { To repeat, in this document the term "restoration" generally refers to any or all of the five fundamental } \\
\text { restoration approaches commonly reported in the literature: creation, enhancement, restoration, conservation, and } \\
\text { protection. When used to refer specifically to restoration as a particular strategy, we will italicize the word; } \\
\text { otherwise, assume the usage in the general sense. }\end{array}$} \\
\hline
\end{tabular}




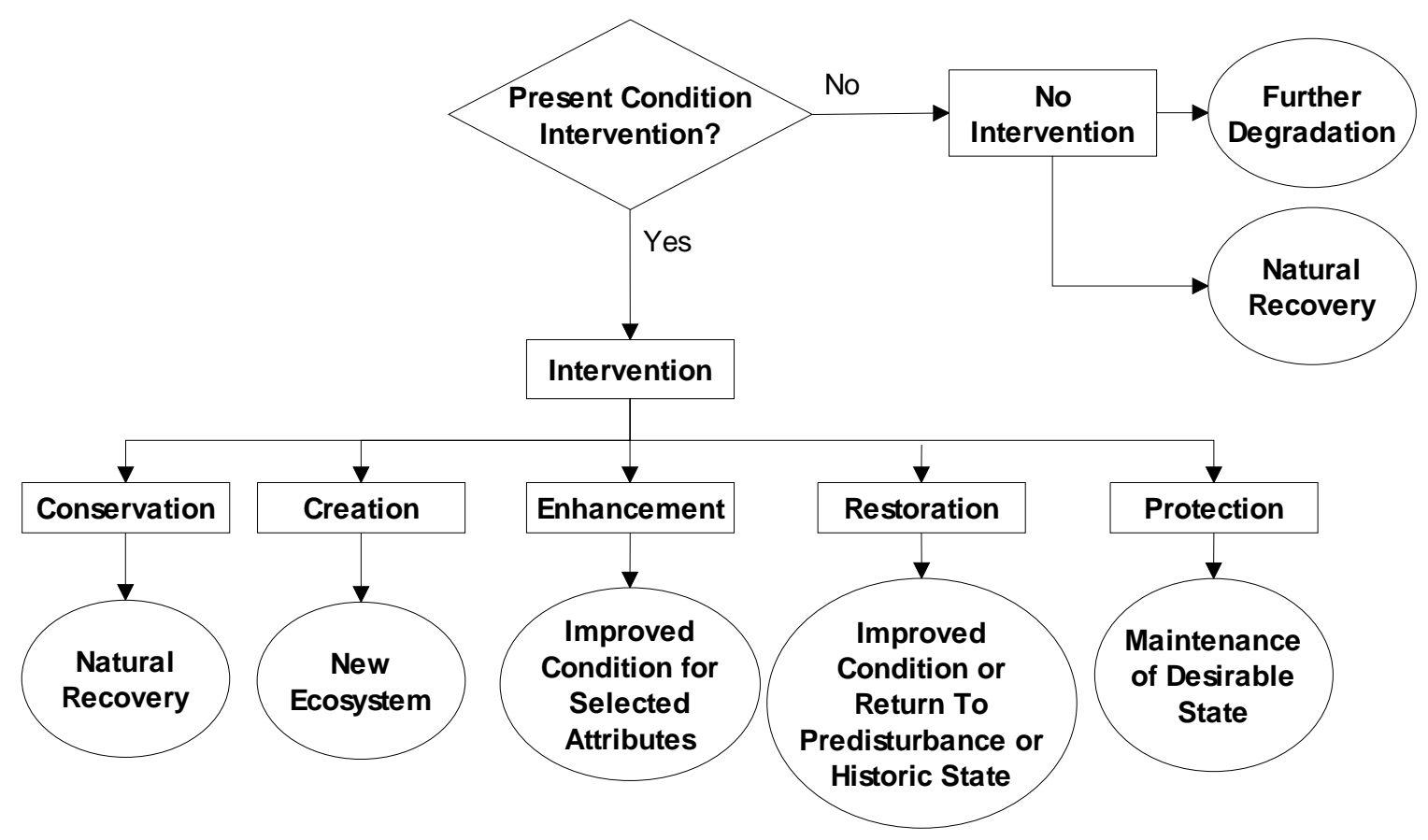

Figure 2.2. Flow Chart of Restoration Pathways (modified from Shreffler and Thom 1993).

No Intervention: In the no intervention approach, recovery is left entirely to natural processes. The outcome of this approach is unpredictable and may not resemble pre-disturbance condition (Class D restoration, Cairns 1991). The two possible trajectories of the no-intervention approach are natural recovery or further degradation. Although represented as two distinct trajectories, further degradation may lead to an alternative steady state, which in turn would progress toward natural recovery. Natural recovery is difficult to grasp because it rarely happens within the lifetime of a scientist or estuary manager and can really only be understood in terms of geological time.

Conservation for Natural Recovery: Conservation can be a practical and effective restoration approach. Conservation biology acknowledges that development of the nearshore and the adjacent upland has and will continue to occur. However, conservation is based on the premise that this development can be done in a way (e.g., using science-based development strategies) to minimize or avoid damage to the biodiversity of the system. Conservation represents a relevant approach for the CRE because there are portions of the system that are highly viable components remaining in the ecosystem, and there will continue to be pressure to develop the region surrounding the system. What is lacking to implement a conservation approach is fundamental information on the relationship between levels and types of development and their impact on biodiversity.

Creation of New Ecosystem: Creation of a new ecosystem involves bringing into being a new ecosystem that previously did not exist on the site (NRC 1992). This approach generally involves "implanting” a created habitat into a coastal shoreline where this type of habitat did not exist previously (Simenstad and Thom 1992). The goal of this approach is to emulate the present condition of an existing, functioning reference ecosystem. Creation of a new ecosystem involves elaborate reconstruction of both physical (e.g., topographic, hydrologic) as well as biotic (e.g., vascular plants) elements. Although created ecosystems may eventually become self-maintaining, there is considerable uncertainty in the outcome. Created ecosystems typically require ongoing management (Class C restoration, Cairns 1991; Simenstad and Thom 1992). 
Enhancement of Selected Attributes: Attributes are characteristics that are correlated with and can serve as indicators of ecosystem structure and function. We have adopted the NRC (1992) definition of enhancement to mean any improvement of a structural or functional attribute. The NRC report (1992) highlighted the importance of considering both structural (state) and functional (process) attributes at population, community, ecosystem, and landscape levels as appropriate. In the Pacific Northwest, the Estuarine Habitat Assessment Protocol (Simenstad et al. 1991) was among the first scientific documents to promote an attribute-oriented approach to assessment of restoration, enhancement, and creation of estuarine habitats. Table 2.1 showed a sample list of selected structural and functional attributes for estuarine systems. As noted by Simenstad and Thom (1992), the probability of successful enhancement is greater if we first understand what processes are required to sustain the integrity of the attributes. Enhancement differs from restoration because only one or several attributes are improved rather than the whole system. Estuarine habitat attributes can be integrated as elements of modified habitats of urbanized estuaries and might increase fish and wildlife function despite the fact that they were not operating within the matrix of a natural habitat (Simenstad and Thom 1992).

Restoration to Improved, Pre-Disturbance, or Historical Condition: Intervention through restoration is intended to improve the existing condition to any degree. Pre-disturbance condition is the condition thought to have previously existed in the estuary prior to the onset of disturbance. From a practical standpoint, pre-disturbance condition is difficult to define precisely and is commonly referred to in the literature as the original, undisturbed condition (Jordan et al. 1997; NRC 1992; Cairns 1989). Historic condition is the condition known to have previously existed in the estuary from historic or recent paleoecological research. The goal of restoration to historic condition is to establish a community that is ecologically superior to the present degraded system and resembles the original system in certain carefully defined ways (Cairns 1988). Simenstad and Thom (1992) note that the opportunity for successful restoration to historic condition is high as long as the primary processes delineating the habitat type are still effective at that site (e.g., salinity intrusion, sedimentation sources and processes, corridors to other natural estuarine and upland habitats). If some, or all, of these processes have been altered or lost, the prospects for restoration to historic condition are greatly diminished.

Protection to Maintain a Desirable State: Although indirect, protection can be an effective intervention tool. Protection helps prevent degradation of existing areas that are presently in a desirable ecosystem state. Protection is distinct from conservation because protection assumes no further development, whereas conservation does not.

\subsubsection{Influence of Disturbance}

The success of a restoration project will vary depending on the level of disturbance (anthropomorphic or natural) of the site and the landscape within which the site resides (NRC 1992). Using the findings of the National Research Council and a review of the literature on estuarine habitat restoration, Shreffler and Thom (1993) concluded that the strategies of restoration, enhancement, and creation should be applied depending on the degree of disturbance of the site and the landscape (Figure 2.3). For example, sites with a high degree of disturbance on both scales, in general have a low probability for restoration, and creation of a new habitat or ecosystem or enhancement of selected attributes would be the only viable strategies to apply in these situations. In contrast, where the site and landscape are essentially intact, restoration to historical (i.e., humans present, but insignificant disturbance) or pre-disturbance (i.e., before man) conditions would be viable options and the probability of success would be high. 


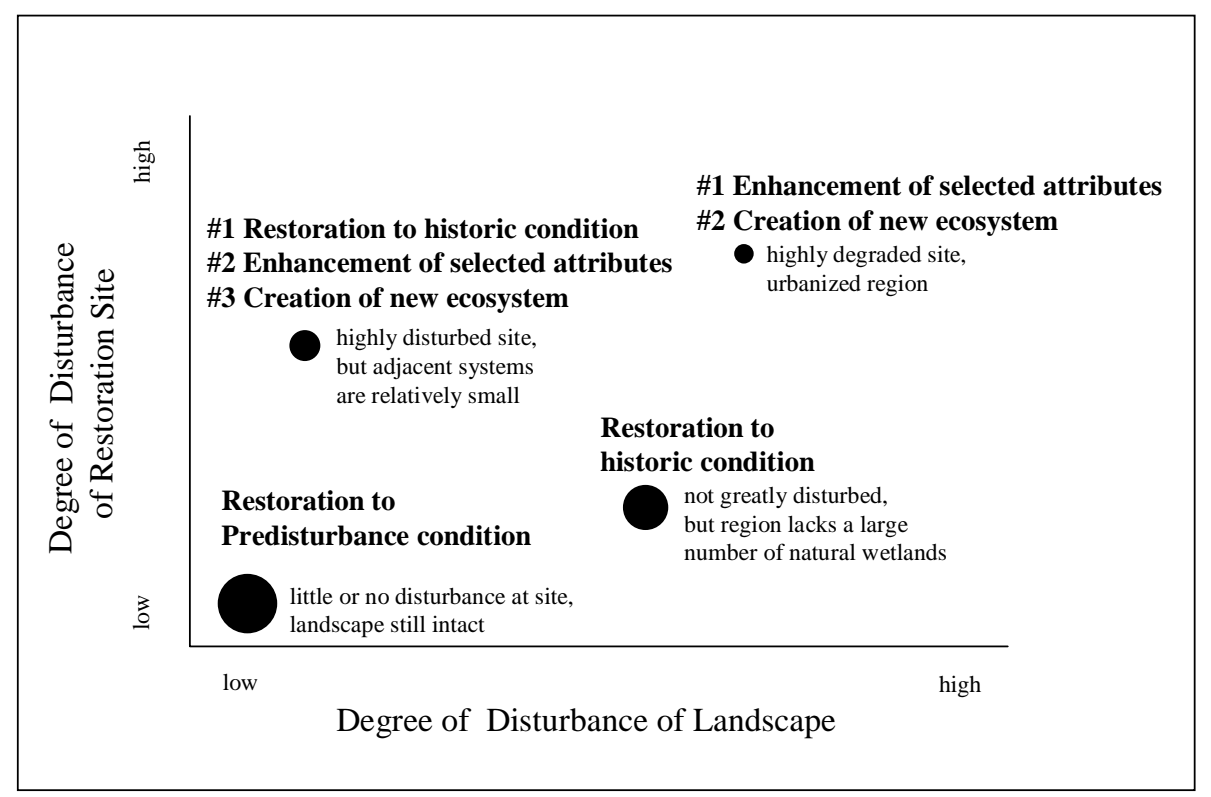

Figure 2.3. Restoration Strategies for Estuarine Systems Relative to Disturbance Levels at the Site and in the Landscape (from Shreffler and Thom 1993). The relative chance of success increases with the size of the dot.

Of the six strategies for restoration presented above (Section 2.1.3), enhancement is well suited to restoration of target species (i.e., individual injured species). Injured species or species groups could benefit directly from enhancement of structural or functional attributes. Watersheds contain arrays of habitats and sites particularly suited for certain species. In many cases, species may be adapted to a unique set of environmental processes or conditions at a particular site that at some time provide a refuge for a species during a period of stress. On the other hand, opportunistic site use allows many species to exist in sites generally not adequate for them. Thus, the importance of a single site for maintaining regional biodiversity is variable--ranging from highly critical during years of restricted habitat to redundant during years of expanded habitat (NRC 1992). Marginal (redundant) sites during one set of conditions may be the best or the only sites when conditions change, but these sites have often been converted to another habitat and are not available when needed. Thus, enhancement actions must be developed with a landscape perspective--an understanding of how specific sites are related to the remaining resources in the watershed or region (NRC 1992). In addition, hypotheses must be formulated, which can be tested by scientifically rigorous methods, about the functions specific habitats or attributes may perform for the target species of concern.

The principal drawback of enhancement is that it is less likely to be self-maintaining than restoration, because only certain attributes at a particular site are re-established or improved rather than the whole ecosystem. Also, from a landscape ecology perspective, enhancement tends to be piecemeal and thus is not the best approach for reducing habitat fragmentation and reintegrating degraded habitats into ecologically optimal locations within the estuary.

The CRE can be classified as moderately disturbed on the landscape scale because of altered hydrology, loss of tidal wetlands, and altered bathymetry. Sites in the Portland/Vancouver area would likely be considered heavily altered, whereas sites in the Cathlamet Bay area might be considered low to moderately disturbed. Strategies in the Portland/Vancouver area would be limited to enhancement of 
selected attributes (e.g., making pocket wetlands larger and more productive) and creation of new ecosystems (e.g., excavating out new wetland habitat). In contrast, restoration in the Cathlamet Bay region might best be accomplished through restoring tidal marshes known to have existed in the region historically.

\subsection{Conceptual Ecosystem Model}

\subsubsection{Background and Objective of a Conceptual Model}

"Conceptual models help to clarify loose thoughts about how a system is composed and how it operates" (Huggett 1993). Conceptual models have been used widely in ecology to depict ecosystems and food webs (e.g., Colby 1978; Odum 1988; Odum and Hornbeck 1997). The National Research Council (NRC 2000) relied on a conceptual model in developing recommendations for a national ecological monitoring program. They used the model to identify and justify key metrics to be monitored. Although not graphically developed, Simenstad et al. (1991) used a conceptual approach to develop environmental monitoring protocols for Puget Sound. Their approach identified measurable characteristics (termed attributes) of estuarine habitats that promote fish and wildlife utilization and fitness. The attributes were selected based on a rigorous assessment (using literature and regional experts) of factors proven to serve these functions. Simenstad et al. (1991; page viii) state that the approach is "designed to accrue information for developing more successful approaches to habitat restoration." Further, they conclude (page 1) that, "neither science nor the technology of estuarine habitat restoration can progress until we treat restoration as rigorous experimentation in which consistent data are gathered in a [n] inductive format." An organized understanding of a system facilitates the inductive process. Finally, Thom (1997; 2000) proposed that conceptual models are a key component of an adaptive management program associated with coastal ecosystem restoration projects and recommended them for planning projects, evaluating the effectiveness of the restoration, providing guidance on adjustments to improve projects success, and refining the understanding of the system being restored.

As has been done for planning restoration of large ecosystems (e.g., Gentile et al. 2001), a conceptual model (or set of models) can significantly help to organize the available information on the CRE that pertains to restoring ecosystem function. The objective of a conceptual model is to contribute to the determination of what needs to be restored, why, and perhaps where the restoration might be most effective. The conceptual model is used to identify the connection between the restoration actions (e.g., physical manipulations) and the physical and biological reactions to such actions, based on the best available information on qualitative and conceptual relationships. As recommended (Simenstad et al. 1991; Thom 2000), a conceptual model is a "living" entity that should be refined and revised as new insight and interpretation becomes available. A conceptual can be one of many tools to aid decisionmakers.

Most conceptual models consist of a simple set of diagrams that illustrate the relationships among the various components of the ecosystem and highlight the more important linkages for the model output (e.g., restoration of natural ecosystem structure and function). In addition to graphically displaying the ecosystem, a model provides a guide for determining what types of data may be most important in understanding long-term component relationships and could be gathered during a monitoring program. The premise of the model is that controlling factors (e.g., hydrology, elevation, sediment type, wave 
energies) result in the development of the habitat structure (i.e., the species and their abundance). In addition, once the habitat structure is developed, the functions (e.g., primary production, food web support, fish and invertebrate rearing and reproduction, organic matter export) normally associated with that structure would be supported. The general format of the model is as follows:

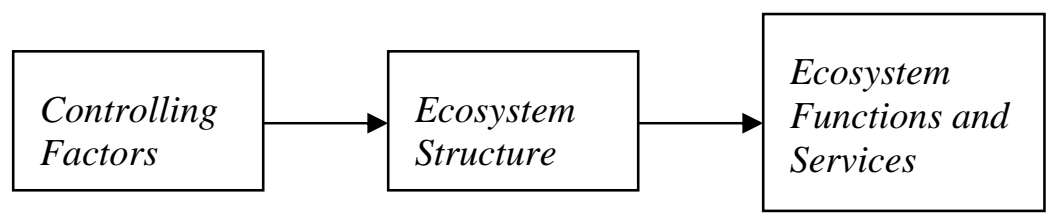

\subsubsection{A Conceptual Model for the CRE}

A conceptual model has been developed for the CRE that has as its endpoint juvenile salmon survival and growth. This model was developed as part of the Biological Assessment of the effects of the COE’s Columbia River Channel Improvement Project and is reproduced in Appendix B. The model utilizes the latest understanding from research and monitoring being conducted on the CRE, including Bottom et al. (2001) and Proctor et al. (1980). The conceptual model in Appendix B can form the basis for determining what factors need to be addressed when developing habitat restoration plans for salmonids. It also provides the basis for factors to consider to maintain restored habitats in the system.

The CRE model can be used as a framework for making decisions in the restoration planning process. By examining the connections between boxes in the model one can determine if the restoration action proposed will directly improve habitat or ecosystem functions. The model also provides key sets of information on what factors must be present to allow a system to develop. For example, are the salinity, elevation, wave energies and sediment input available and adequate to support the development of wetland attributes? Figure 2.4 summarizes the habitat forming processes active in the CRE. These processes result in the types and distribution of habitats in the system. Development and maintenance of these habitats is dependent on the restoration of these processes. If processes such as sediment erosion and accretion, sediment supply, tidal factors, currents, freshwater discharge, and nutrient supply have been degraded or negatively altered past some threshold, a habitat will not develop naturally, and most importantly will not be maintained. In determining the type, size, and configuration of a habitat to be restored, the processes that will allow natural development and maintenance must be considered. In order to benefit salmon, there must be access to the site or the materials (e.g., organic matter, prey resources) must be otherwise available to juvenile salmon through transport to areas where they can access and utilize them.

Figure 2.5 illustrates the relationship between habitats and juvenile salmon in the CRE as developed by Salmon at River's End (SARE) (Bottom et al. 2001). This model indicates that survival and growth of salmonids is dependent on their ability to access habitats that contain ample food resources, and that provide resting (low current) areas and refuge from predation. This element and others from the conceptual model in Bottom et al. (2001) were incorporated into the model in Appendix B. 


\section{Habitat Forming Processes Submodel}

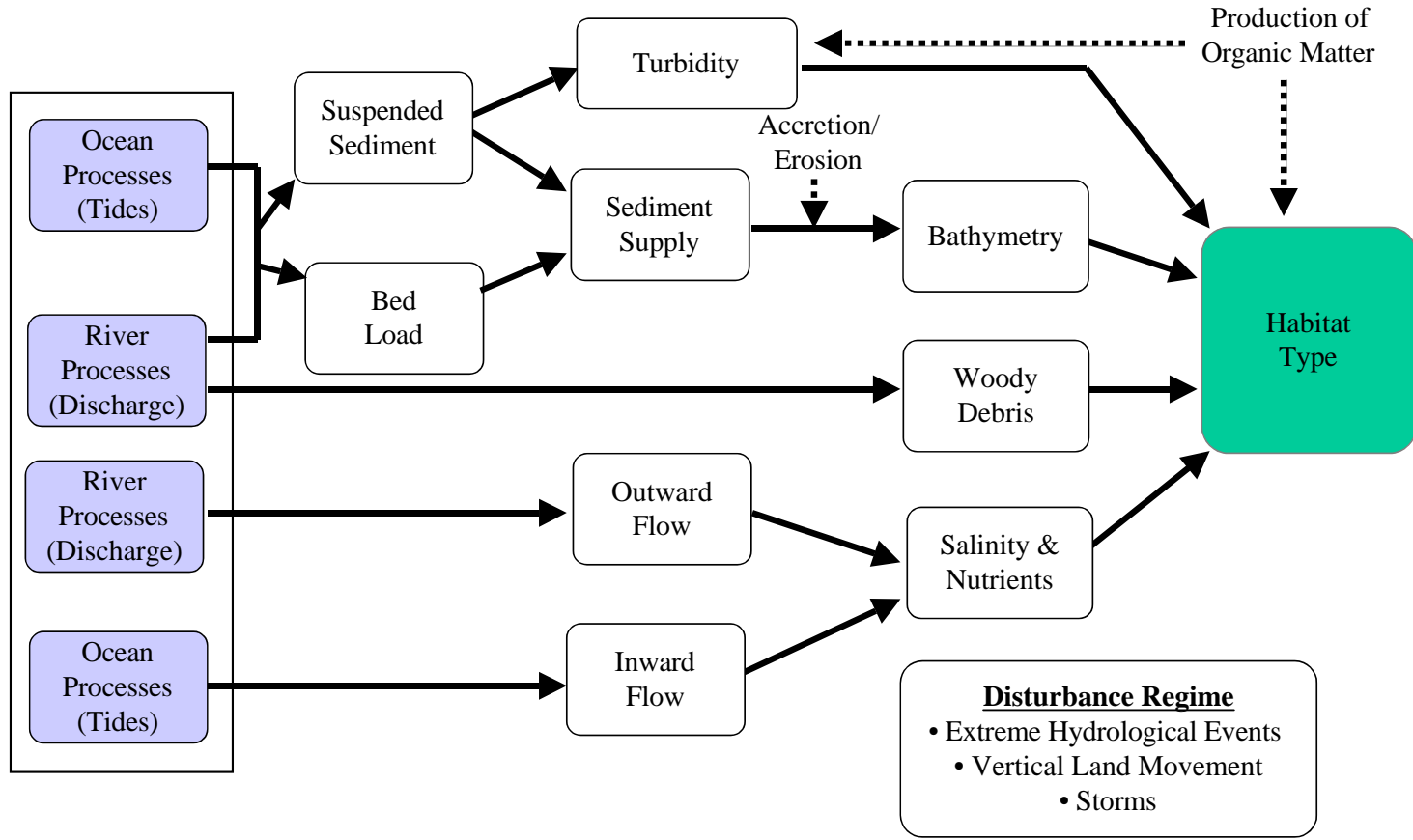

Note: Sediment re-suspension can increase potential for contaminant transport if sediments were contaminated

Figure 2.4. Conceptual Model of Habitat Forming Processes for the CRE (from COE 2001).

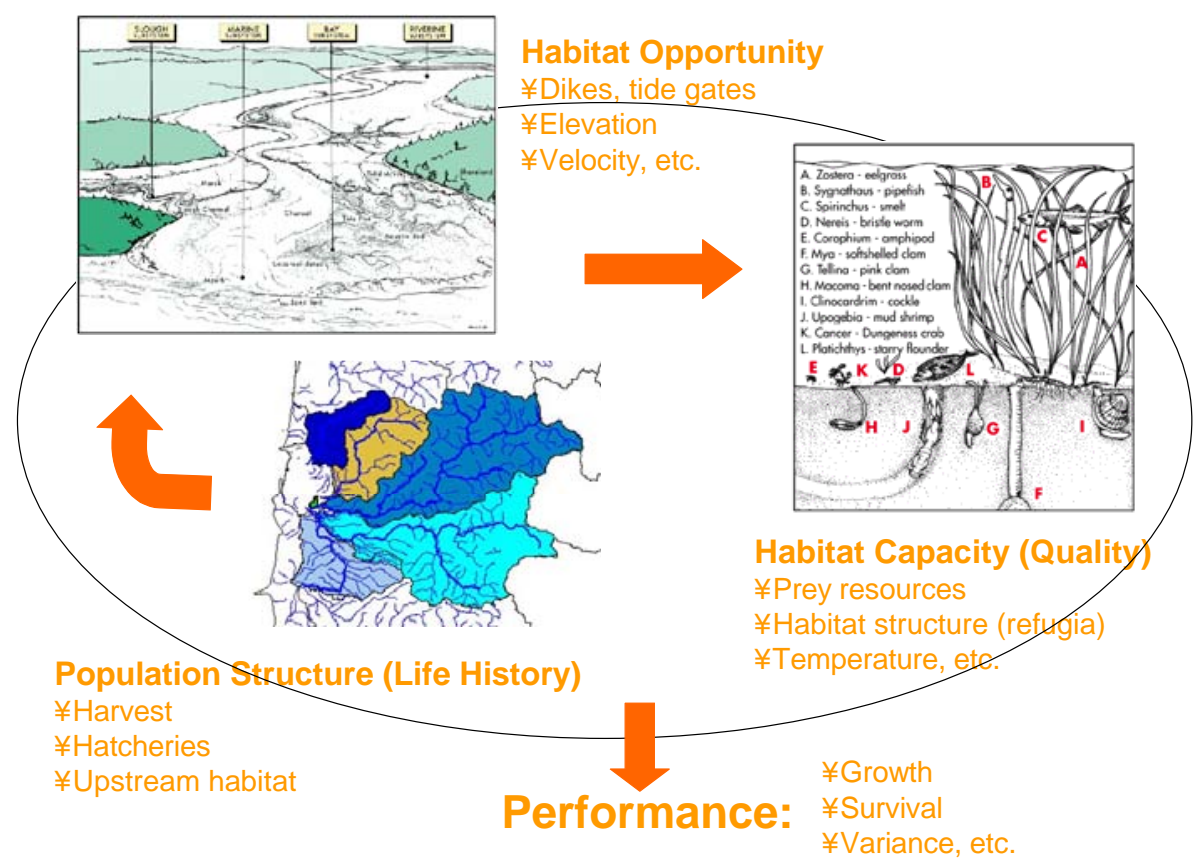

Figure 2.5. Conceptual Model for Juvenile Salmon Growth and Survival (from Bottom et al. 2001). 


\subsection{Juvenile Salmon Usage of the CRE}

The CRE ecosystem is a highly dynamic, structurally complex mix of habitats that form a variety of different environments for juvenile salmonids. The purpose of this section is to review and summarize the existing knowledge of juvenile salmonid usage of the CRE. We base much of this discussion on two recent comprehensive review reports on salmonids in the CRE (Bottom et al. 2001; COE 2001) and on NOAA Technical Memorandums and Status Reviews on salmonid species (Johnson et al. 1999; Johnson et al. 1997; Gustafson et al. 1997; Busby et al. 1996; Hard et al. 1996; Weitkamp et al. 1995). This section describes the available literature on salmonid species present in the estuary, their life history strategies, their physical habitat requirements while residing or migrating through the estuary, and their prey resources. The section closes with a summary and a list of critical uncertainties in the knowledge base for salmon usage and habitat requirements in the CRE.

\subsubsection{CRE Salmonid Species}

In the CRE, there are 60 defined wild or hatchery stocks of salmon and steelhead (Washington State Department of Fish and Wildlife [WDFW] 1992). These include spring and fall Chinook (Oncorhynchus. tshawytscha), fall chum (O. keta), Coho (O. kisutch), and summer and winter run steelhead (O. mykiss). Other salmonid species present in the lower Columbia River estuary include Dolly Varden / bull trout (Salvelinus malma and confluentus) and coastal cutthroat trout (O. clarki) (WDFW 2000). However, juveniles from salmonid stocks upstream must also be considered, as they will also eventually spend some amount of time in the lower Columbia River estuary. There are 64 hatcheries in the states of Washington, Idaho, and Oregon that collectively produce juvenile Chinook (spring and summer), chum, Coho, and steelhead (WDFW 2000). Historically, all salmonids in the Columbia River system were native "wild" fish produced in natural streams, but currently artificial propagation accounts for approximately three-quarters of all returning salmonids. In the Columbia River system there are currently two endangered stocks and 11 threatened stocks listed by Evolutionary Significant Units (ESU) described by the Technical Recovery Teams (TRT) of the National Marine Fisheries Service (NMFS 2003) (Table 2.4).

Table 2.4. Technical Recovery Team, Evolutionarily Significant Unit, and Endangered Species Act (ESA) Listing under the Federal Columbia River Power System Biological Opinion.

\begin{tabular}{lll}
\hline Technical Recovery Team & Evolutionarily Significant Unit & ESA Listing \\
\hline Willamette and Lower Columbia & Lower Columbia River Chinook & threatened \\
River & Upper Willamette River Chinook & threatened \\
& Columbia River Chum & threatened \\
& Lower Columbia River Steelhead & threatened \\
\hline Interior Columbia River Basin & Upper Columbia River Spring Chinook & endangered \\
& Snake River Spring/Summer Chinook & threatened \\
& Snake River Fall Chinook & threatened \\
& Upper Columbia River Steelhead & endangered \\
& Mid Columbia River Steelhead & threatened \\
& Snake River Steelhead & threatened \\
& Snake River Sockeye & endangered \\
\hline
\end{tabular}




\subsubsection{Life History Strategies}

Because this approach is geared toward the restoration of habitat that will benefit juvenile salmonids, we review the various life history types that have evolved and the different habitats used by each. Juvenile salmon have several different life history strategies to cope with the physical variations in a given system. Currently in the CRE, there are two general life history strategies - "ocean-type" and "stream-type" - with different habitat requirements among them (COE 2001). However, there is some ambiguity in our understanding and definition of these life history strategies. For example, Rich (1920, cited in Bottom et al. 2001) described several forms of ocean-type life histories for Chinook salmon based on fork length, time of collection, and scale analysis. Supporting this notion of diversity within a general life history strategy, Wissmar and Simenstad (1998) estimated that there might be as many as 35 potential variations on the ocean-type life-history strategy for Chinook salmon. These variations reflect genotypic and tactical responses to the estuarine system that have evolved during post-glacial variations in river flow, landscape, and sea level geomorphologies and biogeographic variation. Optimum strategies maximize life history diversities to accommodate the range of conditions (Simenstad and Cordell 2000). Furthermore, juvenile life history appears to be a heritable trait (Myers 1998), and stream-type life history appears to be recessive to ocean-type life history (Clarke and Shelbourn 1985). A brief overview of the characteristics of the two most common general life history types is in Table 2.5.

Table 2.5. Salmon Life History Comparison (from COE 2001)

\begin{tabular}{lll}
\hline Characteristic & Ocean type & Stream-type \\
\hline Species Composition & Includes fall Chinook chum & $\begin{array}{l}\text { Includes spring Chinook, Coho, } \\
\text { sockeye, steelhead, and cutthroat } \\
\text { trout }\end{array}$ \\
Freshwater Residence Time & $\begin{array}{l}\text { Rear only weeks to months in fresh } \\
\text { water }\end{array}$ & $\begin{array}{l}\text { Rear more than 1 year in fresh water } \\
\text { prior to downstream migration }\end{array}$ \\
Size & $\begin{array}{l}\text { Small (30 to } 80 \mathrm{~mm}) \\
\text { Large (100 to 300 mm or larger) }\end{array}$ \\
Habitat Preference & $\begin{array}{l}\text { Use shallow water/shoreline habitat } \\
(0.1 \text { to } 2 \text { meters deep, current less }\end{array}$ & $\begin{array}{l}\text { Generally move in open water and } \\
\text { main channels }\end{array}$ \\
& $\begin{array}{l}\text { than } 0.3 \text { meters per second) } \\
\text { Prolonged rearing in lower river } \\
\text { CRE Residence Time }\end{array}$ & $\begin{array}{l}\text { Move relatively quickly through } \\
\text { lower river (days to weeks) }\end{array}$ \\
\hline
\end{tabular}

\subsubsection{Temporal Distribution and Residence Time}

Juvenile salmonids are likely found in the lower Columbia River estuary during the entire year, depending on species, size class, and life history type, with large numbers of juveniles first appearing in middle to late March (COE 2001). A variety of size classes can be found migrating through the CRE mostly from March through October.

Ocean-type Chinook salmon tend to spend more time than stream-type Chinook salmon in estuaries for rearing and move relatively slowly through the estuary, remaining primarily in shallow water habitats (Bottom et al. 1984). Ocean-type juveniles (Chinook and chum salmon) from tributaries in the lower Columbia River start migrating several months after emergence in the early spring, while upriver oceantype fall Chinook stocks migrate over Bonneville Dam with peak numbers in early July (COE 2001). Chum salmon fry generally emerge from the gravel during February-June and migrate to saltwater soon after emergence in the spring. This downstream migration ranges from a few hours to several days 
depending on the location of emergence relative to the estuary (Johnson et al. 1997). Chum salmon ocean-type juveniles are the youngest and smallest juvenile salmon found in the estuary (COE 2001). Ocean-type salmon are more dependent on estuaries than stream-type salmon (Thorpe 1994).

Stream-type juvenile Chinook are much more dependent on freshwater for rearing because of their extended residence times in freshwater. They typically spend one year in freshwater before migrating to saltwater in the spring. Included in the stream-type life history are Coho salmon, sockeye salmon, both winter and summer run steelhead, coastal cutthroat trout, and Dolly Varden / bull trout. Coho juveniles spend approximately one year in freshwater before migrating to the ocean, with peak outmigration occurring in May (WDFW and ODFW 2002, Weitkamp et al. 1995; Durkin 1982). Natural production of juvenile Coho salmon is less than $10 \%$ of Columbia River Coho salmon, with the majority produced from hatcheries (WDFW and ODFW 2002; Durkin 1982). Juvenile sockeye normally rear for at least one full year in a freshwater lake before moving to the estuarine environment in the Columbia River system and are the southernmost sockeye runs (Wenatchee and Okanogan) in North America (WDFW and ODFW 2002). The anadromous runs of sockeye begin to migrate downstream in mid- to late-May, although Chapman et al. (1995, in Gustafson et al. 1997) point out that the arrival of smolts at lower Columbia River dams is earlier now than was reported from the 1940s through the 1960s. Both winter and summer wild steelhead runs migrate in May-June after two to three years in freshwater. Hatchery steelhead smolts also migrate downstream at the same time, but usually after only one year of freshwater rearing (WDFW and ODFW 2002). Bull trout emerge in late winter or early spring and, in the case of coastal populations such as the lower Columbia River, may stay in the area of emergence or migrate to the saltwater within a few weeks upon emergence (USFWS 1998).

\subsubsection{Spatial Distribution and Physical Habitat Characteristics}

Juvenile salmonid habitat requirements are comprised of several controlling factors and structures that support a particular life history or species. This section and the review of the physical habitat requirements for juvenile salmonids focuses primarily on the two dominant life history types (ocean-type and stream-type) associated with those species listed in (Table 2.4), especially those species that have been identified as estuarine-dependent such as ocean-type Chinook and chum (Bottom et al. 2001; Myers et al. 1998; Johnson et al. 1997; Bottom et al. 1984).

As stated in COE (2001), "The quality or suitability of habitat meeting the needs of an organism of concern is determined by a variety of factors." Stream stability, flow, temperature, estuary productivity, general weather patterns, and distance to the marine environment are thought to be key factors in the evolution and expression of the temporal distribution of the downstream migration for juvenile Chinook salmon (Myers et al. 1998). The physical habitat requirements of juvenile salmonids are related to their life history pattern. The primary factors that describe physical habitats are water depth, water velocity, and substrate type, while secondary physical factors are water temperature, salinity, and turbidity (COE 2001). The information presented in Table 2.6 summarizes the physical habitat requirements for juvenile salmonids in the Columbia River estuary (D. Weitkamp, pers. comm. to R. Thom, May 2001).

Typically, ocean-type juveniles have more stringent physical habitat requirements than stream-type juveniles, although wide ranges of acceptability are often observed (Table 2.6). Ocean-type juvenile salmonids are small subyearling fish that are generally found oriented to shorelines in shallow water habitats, whereas stream-type Chinook salmon are most often found in channel areas (Bottom et al. 2001; COE 2001; Johnsen and Sims 1973). 
Table 2.6. Physical Habitat Requirements for Juvenile Salmon in the CRE.

\begin{tabular}{|c|c|c|}
\hline Parameter & Ocean-Type & Stream-Type \\
\hline Water Depth & Surface waters & Surface to $10 \mathrm{~m}$ \\
\hline \multirow[t]{2}{*}{ Currents } & Less than $\sim 9 \mathrm{~cm} / \mathrm{second}$ & \multirow{2}{*}{$\begin{array}{l}\text { Larger fish than ocean-type, hence } \\
\text { found throughout a much wider range } \\
\text { of current velocities and tend to avoid } \\
\text { low-velocity areas }\end{array}$} \\
\hline & $\begin{array}{l}\text { Less than } 30 \mathrm{~cm} / \mathrm{second}^{(1)} \text { for current } \\
\text { threshold modeling }\end{array}$ & \\
\hline Substrate & Varies (mostly sand/silt) & $\begin{array}{l}\text { Varies, but tends to be associated with } \\
\text { the water column to a greater extent } \\
\text { than with substrate type }\end{array}$ \\
\hline \multirow[t]{3}{*}{ Salinity } & Upon hatching 15-20 ppt ${ }^{(2)}$ & \multirow[t]{3}{*}{ Generally same as ocean-type } \\
\hline & Juveniles 30 ppt seawater ${ }^{(3)}$ & \\
\hline & $\begin{array}{l}\text { Chinook fry of } 1.5 \text { gram could survive and } \\
\text { grow in seawater }{ }^{(4)}\end{array}$ & \\
\hline \multirow[t]{2}{*}{$\begin{array}{l}\text { Water } \\
\text { Temperature }\end{array}$} & $\begin{array}{l}\text { Can tolerate brief periods of } 15-20^{\circ} \mathrm{C} \text { Lethal } \\
\text { at approximately } 22^{\circ} \mathrm{C}^{(5)}\end{array}$ & \multirow[t]{2}{*}{ Generally same as ocean-type } \\
\hline & $\begin{array}{l}\text { Sub-lethal effects can occur below lethal } \\
\text { threshold, but vary. }\end{array}$ & \\
\hline \multirow[t]{2}{*}{ Turbidity } & $\mathrm{LC}_{50}$ for Coho (summer conditions) $1.2 \mathrm{~g} / \mathrm{l}$ & \multirow[t]{2}{*}{ Generally same as ocean-type } \\
\hline & $\underset{(7)}{\mathrm{LC}_{50}}$ for chum (summer conditions) $2.5 \mathrm{~g} / \mathrm{l}$ & \\
\hline
\end{tabular}

(1) Bottom et al. 2001, (2) Wagner et al. 1969, (3) Tiffan et al. 2000, (4) Clark and Shelbourn 1985,

(5) Brett 1971, Lee and Rinne 1980, (6) Noggle 1978, (7) Smith 1978

Although stream-type juvenile salmonids can be found throughout the estuary in a variety of habitats and depth ranges (0.3-10 meters), they have a propensity to be found near the water surface. They are capable of utilizing higher current velocity areas and tend to avoid low velocity areas except when holding position against tidal currents or river currents. They also tend to be associated with mid-channel more than with the shoreline and therefore are typically not so closely associated with specific substratum types. The smoltification process of stream-type juveniles usually begins prior to downstream movement; however, these fish are robust in their ability to adapt to saline environments very rapidly.

Historically, large woody debris from upland forests probably played an important role as structure in estuarine habitats used by juvenile salmon. This landscape-scale input to the CRE has declined over the years. The resultant effect on CRE rearing habitat for juvenile salmon is not well understood.

\subsubsection{Prey Resources}

There is limited information regarding the prey resources for juvenile salmonids in the CRE. Most data relates to specifically Chinook and chum salmon. However, two studies (Craddock et al. 1976; Bottom and Jones 1990) have documented the feeding by juvenile salmonids (Chinook) on Daphnia. Bottom and Jones (1990) reported that young Chinook ate primarily Corophium, Daphnia, and insects, 
with Corophium being dominant in winter and spring, and Daphnia dominant in summer. Dawely et al. (1986) reported that juvenile salmon in the upper portions of the lower Columbia River consumed insects (diptera, hymenoptera, coleoptera, tricoptera, and ephemeroptera), but in the lower reaches their diets changed to diptera, cladocerans, and amphipods (Corophium salmonis, Corophium spinicorne, Eogammarus confervicolus). In Coos Bay, Oregon, Fisher and Pearcy (1996) found that the diets of juvenile subyearling Chinook salmon (wild) and yearling Chinook salmon (hatchery) overlapped depending on location and size of fish.

Perhaps the most significant effect on the CRE system has been the apparent shift from a macrodetrital- to a microdetrital-based system (Sherwood et al. 1990). The loss of wetlands and emergent plants that formerly contributed to the macrodetritus based system may have significantly affected the carrying capacity of the estuary to support juvenile salmonids (Bottom et al. 2001). This reduction in capacity may in turn be affecting the opportunity of juvenile salmon to access specific habitats in the estuary and associated functions such as foraging (Simenstad and Cordell 2000). However, as stated in Bottom et al. (2001, page 152), “...the intrinsic assumption that food or predation in estuary may limit juvenile salmon productivity, or that there are carrying capacity limitations for juvenile salmon in the Columbia River estuary, has never been rigorously tested." Furthermore, the historical information that has been collected on the diet composition of juvenile salmonids has been biased by a preponderance of large stream-type fish (Bottom et al. 2001).

The variability of juvenile salmonid prey consumed by Chinook (and other juvenile salmonids) varies throughout the literature depending on location and time of year. While there is a fundamental lack of data to support specific prey preferences in the CRE, the inference can be made that CRE juvenile salmonids have the same feeding preferences as those in other estuaries. Johnson et al. (1997) state "...that juvenile salmon, particularly chum and Chinook, depend heavily on benthic organisms in estuaries, but in outer areas they depend more on planktonic organisms.” Simenstad et al. (1983) compared the diets of juvenile salmonids in 16 estuaries and concluded that small juveniles $(<\sim 50-60$ $\mathrm{mm}$ ) feed primarily on epibenthic crustaceans such as harpacticoid copepods, gammarid amphipods, and isopods, while larger juveniles in neritic habitats feed more on drift insects and plankton such as calanoid copepods, larvaceans, and hyperiid amphipods.

\subsubsection{Summary of Habitat Requirements and Uncertainties}

Estuaries and lower river reaches are more than a simple corridor to the ocean for out-migrating salmonids. The CRE is presumed to provide a multitude of ecological functions for a wide array of species (including non-salmonids), and to some degree all of the different life history strategies associated with juvenile salmonids that encompass its vast watershed. The state of knowledge regarding juvenile salmon usage of the CRE ranges from poor to good. We have a good understanding of the various species and a generally good understanding of their life history strategies as well as their temporal distribution. Less well understood is their residence time in the estuary. We know generally that oceantype juveniles are oriented to shoreline and shallow water and tidal marsh and swamp habitats. Specific habitat requirements need further documentation, although it is apparent that tidal wetlands are important to salmonids. Finally, more study is needed regarding prey resources, including epibenthic and planktonic organisms, and knowledge of biological processes needs to be linked to our knowledge of physical habitat characteristics. 
There are deficiencies in the knowledgebase regarding basic ecological processes and linkages between biological and physical functions in the CRE. Bottom et al. (2001) identified fundamental studies to evaluate the link between juvenile salmon and estuarine conditions in the Columbia River. In addition, an "Estuary Research Needs Workshop" was held in March 2003 that identified many important gaps in our knowledge base on juvenile salmon usage of the CRE especially as it relates to habitat restoration. Research has been initiated to perform some of the recommended studies. Consolidating Bottom et al. (2001) and the Research Needs Workshop, the following uncertainties concern salmon habitat requirements in the CRE in the context of ecosystem restoration planning:

- The relationship between life history strategies and habitat requirements, especially for ESAlisted species.

- Juvenile salmon usage and basic ecology in the Tidal Freshwater Subsystem.

- Linkages between biological and physical processes in various habitat types.

- Size, quality, and accessibility of habitat preferred by juvenile salmonids, i.e., the landscape ecology concepts of minimum area, shape, and corridors (see Section 2.1.2).

- Specific growth and survival rates for various life history types and the relationship to habitat type.

- Habitat forming processes required to maintain habitat types utilized by salmon.

- Food web characteristics and linkages.

\subsection{Habitat Changes in the Columbia River Estuary}

Current ecological conditions in the Columbia River estuary reflect over 120 years of anthropogenic impacts that have altered ecosystem inputs and processes, thereby affecting salmon habitat. In this section, we describe what is known about current and historical ecological conditions in the CRE by presenting an analysis of habitat changes compiled from various habitat inventory efforts. This set of information is meant to assist the restoration project planner in applying the general restoration strategies outlined above (Section 2.1.3) and in more targeted restoration project planning under the Implementation Guidelines (Section 3). While limited in terms of detail and spatial-temporal variability, the habitat change information provides an important basis to understand biophysical variables that have altered salmon population structure and phenotypic behaviors. The habitat change analysis will be used to provide context to select and design habitat restoration projects under present conditions.

It is important during the planning of a restoration project to understand how the ecosystem has changed, i.e., where habitats used to occur and their historical natural size, shape, community composition, and connectivity to the other elements of the ecosystem. To this end, we compiled information from various sources (Table 2.7) to help characterize changes in habitat. Existing historical and current habitat comparisons rely on the interpretation of U.S. Coast Survey charts from 1870-1878 done by Thomas (1983) during Columbia River Estuary Data Development Program (CREDDP). Other existing historical datasets include a digitized compilation of Thomas' (1983) work and further interpretation of U.S. Coast Survey charts in the tidal freshwater reach from Puget Island to Portland (Graves et al. 1995). Other historic comparisons include aerial photo interpretations done by the COE that examine habitat type changes from five periods in 1948, 1961, 1973, 1983, and 1991 (COE 1996). 
Currently, historic habitat comparison classification work is being undertaken by NOAA Fisheries. The object of the current work is to reconstruct historic (1868 to 1901) habitat structure from geo-referenced and digitized U.S. Coast and Geodetic Survey topographic survey sheets at a higher spatial resolution than is currently available and to compare it to the 2002 habitat mapping by the Estuary Partnership (Garano et al. 2002).

Table 2.7. Summary of Habitat Mapping Projects for the CRE

\begin{tabular}{|c|c|c|c|}
\hline Project & Date of Survey & Area of Coverage & Citation \\
\hline CREDDP & $1870-1878$ and 1980 & RM 0-46 & Thomas (1983) \\
\hline CREST & $1870-1888$ & RM 0-105 & Graves et al. (1995) \\
\hline $\begin{array}{l}\text { Bi-State Water Quality } \\
\text { Program }\end{array}$ & 1991 & RM 0-146 & Tetratech (1996) \\
\hline C-CAP & 1992 & RM 0-80 & NOAA (1992) \\
\hline $\begin{array}{l}\text { Bi-State Water Quality } \\
\text { Program }\end{array}$ & $\begin{array}{l}\text { 1948, 1961, 1973, } \\
1983,1991\end{array}$ & RM 0-146 & COE (1996) \\
\hline $\begin{array}{l}\text { Floodplain Habitat Cover } \\
\text { Types }\end{array}$ & 2002 & RM 0-146 & Garano et al. (2002) \\
\hline $\begin{array}{l}\text { Characterizing Habitat } \\
\text { Change }\end{array}$ & Ongoing & $\begin{array}{l}\text { Focal areas from RM 0- } \\
146\end{array}$ & $\begin{array}{l}\text { In progress by } \\
\text { NOAA }\end{array}$ \\
\hline
\end{tabular}

\subsubsection{Habitat Types}

It is relevant to examine the major habitat types that historically occurred and to compare them to those in existence today. Five major types of habitat were defined by CREDDP (Thomas 1983) according to dominant vegetation: tidal swamps, tidal marshes, tidal flats, medium depth water, and deep water. Tidal swamps are those areas in which the dominant vegetation is mostly shrub and woody species with elevations varying between mean high high water (MHHW) and the line of non-aquatic vegetation. Tidal marshes vary considerably depending on dominant herbaceous vegetation and have been recorded between mean low low water (MLLW) to slightly above MHHW. Tidal flats are defined as being between the MLLW mark and -6 ft MLLW. Medium depth is between $3 \mathrm{ft}$ and $18 \mathrm{ft}$ below MLLW. Finally, deep water is defined as $18 \mathrm{ft}$ and deeper. In the following sections, for each of the estuarine subareas and the tidal freshwater subsystem, habitat changes are described and a brief characterization of how past impacts may have contributed to existing habitat type structure is presented.

\subsubsection{Subsystems and Subareas}

The study area's subsystems and subareas (Section 1.4) were used to frame the comparison between current and historical conditions. Recall, we divided the CRE study area into two broad subsystems based on salinity: estuarine (RM 0-46) and tidal freshwater (RM 46-142). Then the estuarine subsystem was partitioned into subareas (Figure 2.6) based on physical characteristics (e.g., sediment processes and exposure). 


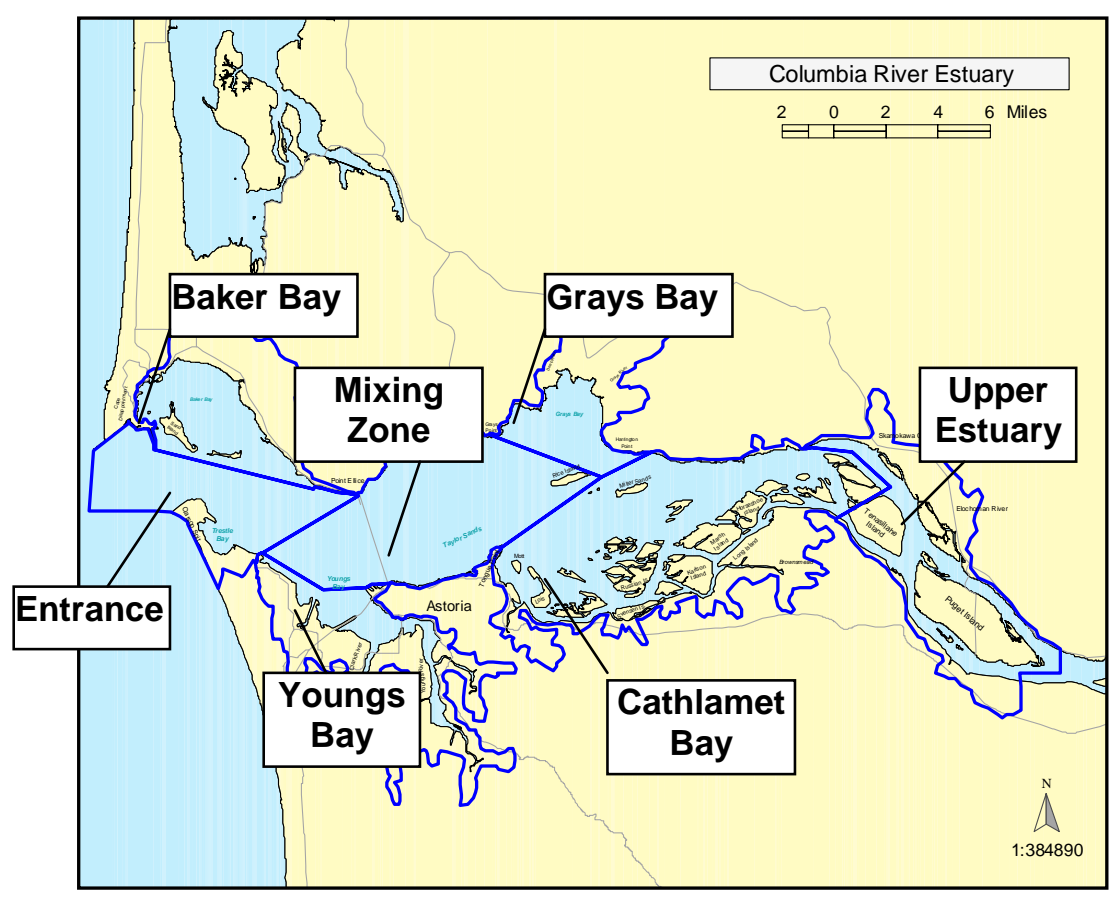

Figure 2.6. Columbia Estuary Subsystem with Seven Subareas

\subsubsection{Entrance Subarea}

Subtidal habitat types dominate the Entrance Subarea (Figure 2.7), which has the highest salinity found in the CRE. Historically this region was a high-energy area of natural fluvial land forms such as the Clatsop Spit, Trestle Bay, and a complex of channels, shallow water, and sand bars. This subarea has undergone dramatic changes since the 1800s (Table 2.8) including the migration of midchannel islands (Sand Island) toward Baker Bay. More abrupt changes have resulted from dredging and jetty construction that limited the ocean-fed supply of sediment entering the area, forming new accretion zones along areas like Clatsop Plains and Long Beach Peninsula. The accumulation of these impacts has manifested in an increase in deep water acreage and a loss of medium-depth habitat and shallow flat habitat types. The Entrance Subarea supports the Columbia Plume, which forms an ecologically significant aquatic 'front' between freshwater flow conditions of the Columbia River and ocean currents.

Table 2.8. Habitat Changes - Entrance Subarea

\begin{tabular}{cccc}
\hline Habitat Type & 1870 Acreage & 1983 Acreage & Change \\
\hline Deep Water & 8,900 & 10,580 & $+18.9 \%$ \\
Medium Depth & 4,480 & 2,640 & $-41.1 \%$ \\
Shallow/flats & 2,980 & 1,680 & $-43.6 \%$ \\
Tidal Marshes & 0 & 250 & N/a \\
Tidal Swamps & 0 & 0 & N/a \\
\hline
\end{tabular}



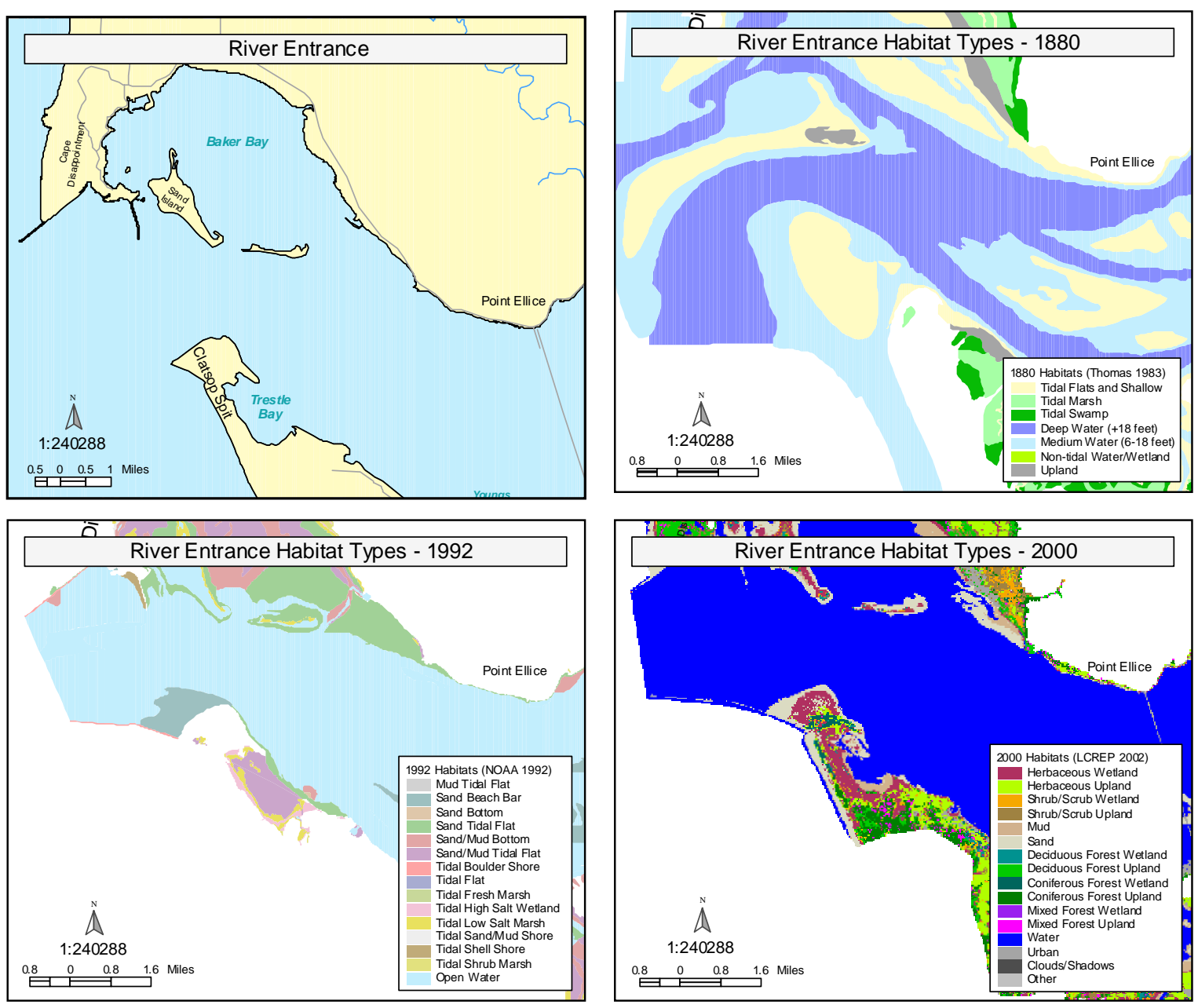

Figure 2.7. Habitat Maps of the Entrance Subarea

\subsubsection{Mixing Zone Subarea}

The mixing zone subarea (Figure 2.8) is characterized by a network of mid-channel shoals and flats, as demonstrated by Desdemona and Taylor Sands. This subarea has the highest variation in salinity resulting from the dynamics between spring/neap tide cycles and river flow. Related to this interaction is the phenomenon known as the estuary turbidity maximum, which is expressed in both the Mixing and Entrance subareas. Fringing subtidal and intertidal habitats have been moderately impacted through the development of Astoria and Washington shoreline along Hwy 404 (Table 2.9). Overall, there has

Table 2.9. Habitat Changes - Mixing Zone Subarea

\begin{tabular}{cccc}
\hline Habitat Type & 1870 Acreage & 1983 Acreage & Change \\
\hline Deep Water & 8,450 & 8,360 & $-1.1 \%$ \\
Medium Depth & 10,780 & 10,330 & $-4.2 \%$ \\
Shallow/flats & 9,540 & 9,490 & $-0.5 \%$ \\
Tidal Marshes & 10 & 10 & $0.0 \%$ \\
Tidal Swamps & 0 & 0 & N/a \\
\hline
\end{tabular}
been relatively little change in total areas of the various habitat types. 

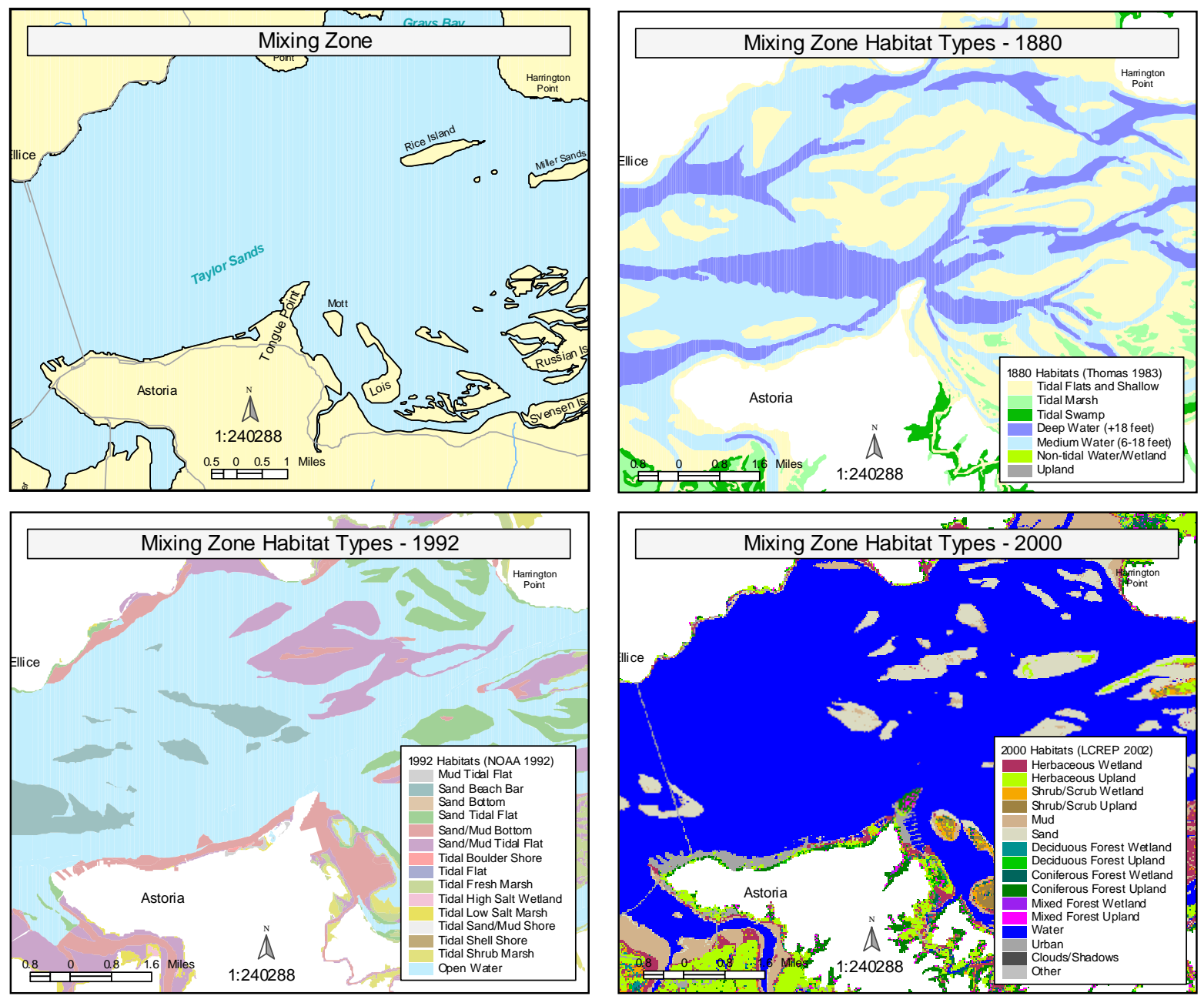

Figure 2.8. Habitat Maps of the Mixing Zone Subarea

\subsubsection{Youngs Bay Subarea}

Youngs Bay is the subarea most impacted by diking and flood control structures in the Columbia River estuary (Table 2.10; Figure 2.9). This area's broad flood plain made it extremely suitable for pasture conversion. The tidal marsh/tidal swamp habitat types are now expressed only in remnant fragmented patches that may serve as a reference for multiple opportunities in restoration planning. Most of the existing tidal marsh areas were recently formed adjacent to dikes and are documented to be slightly different in their physical structure and vegetative community than their historic predecessors.
Table 2.10. Habitat Changes -- Youngs Bay Subarea

\begin{tabular}{cccc}
\hline Habitat Type & 1870 Acreage & 1983 Acreage & Change \\
\hline Deep Water & 810 & 850 & $+4.9 \%$ \\
Medium Depth & 1,120 & 870 & $-22.3 \%$ \\
Shallow/flats & 4,400 & 3,860 & $-12.3 \%$ \\
Tidal Marshes & 7,210 & 980 & $-86.4 \%$ \\
Tidal Swamps & 3,000 & 130 & $-95.7 \%$ \\
\hline
\end{tabular}



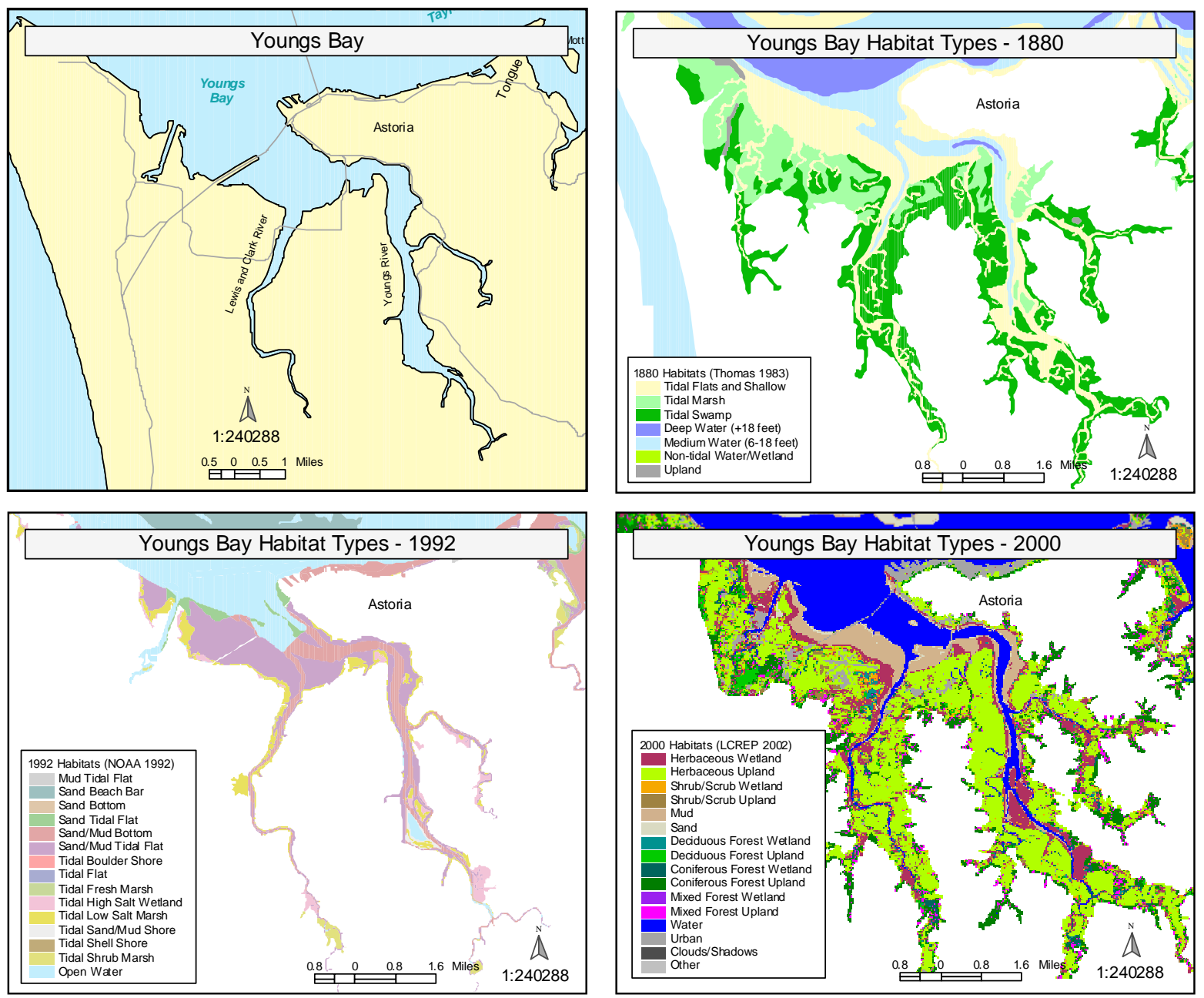

Figure 2.9. Habitat Maps of the Youngs Bay Subarea

\subsubsection{Baker Bay Subarea}

Baker Bay has changed dramatically since the 1870s (Table 2.11; Figure 2.10). Located adjacent to the river mouth, this subarea expresses features associated with brackish water. Formerly a highenergy area of the Columbia River, island migration into the interior of Baker Bay has partially sheltered this area from wave action and currents. By the 1930s, complex tidal slough networks had been drained and diked, effectively cutting off a large portion of the tidal marsh and swamp habitat described above. While significant tidal marsh and swamp habitat has been lost, some tidal marsh has recently started to develop as a result of diminished exposure to waves.
Table 2.11. Habitat Changes - Baker Bay Subarea

\begin{tabular}{cccc} 
Habitat Type & 1870 Acreage & 1983 Acreage & Change \\
\hline Deep Water & 1,800 & 450 & $-75.0 \%$ \\
Medium Depth & 4,700 & 1,350 & $-71.3 \%$ \\
Shallow/flats & 4,830 & 8,450 & $74.9 \%$ \\
Tidal Swamps & 3,480 & 0 & $-100.0 \%$ \\
Tidal Marshes & 1,640 & 730 & $-55.5 \%$ \\
\hline
\end{tabular}



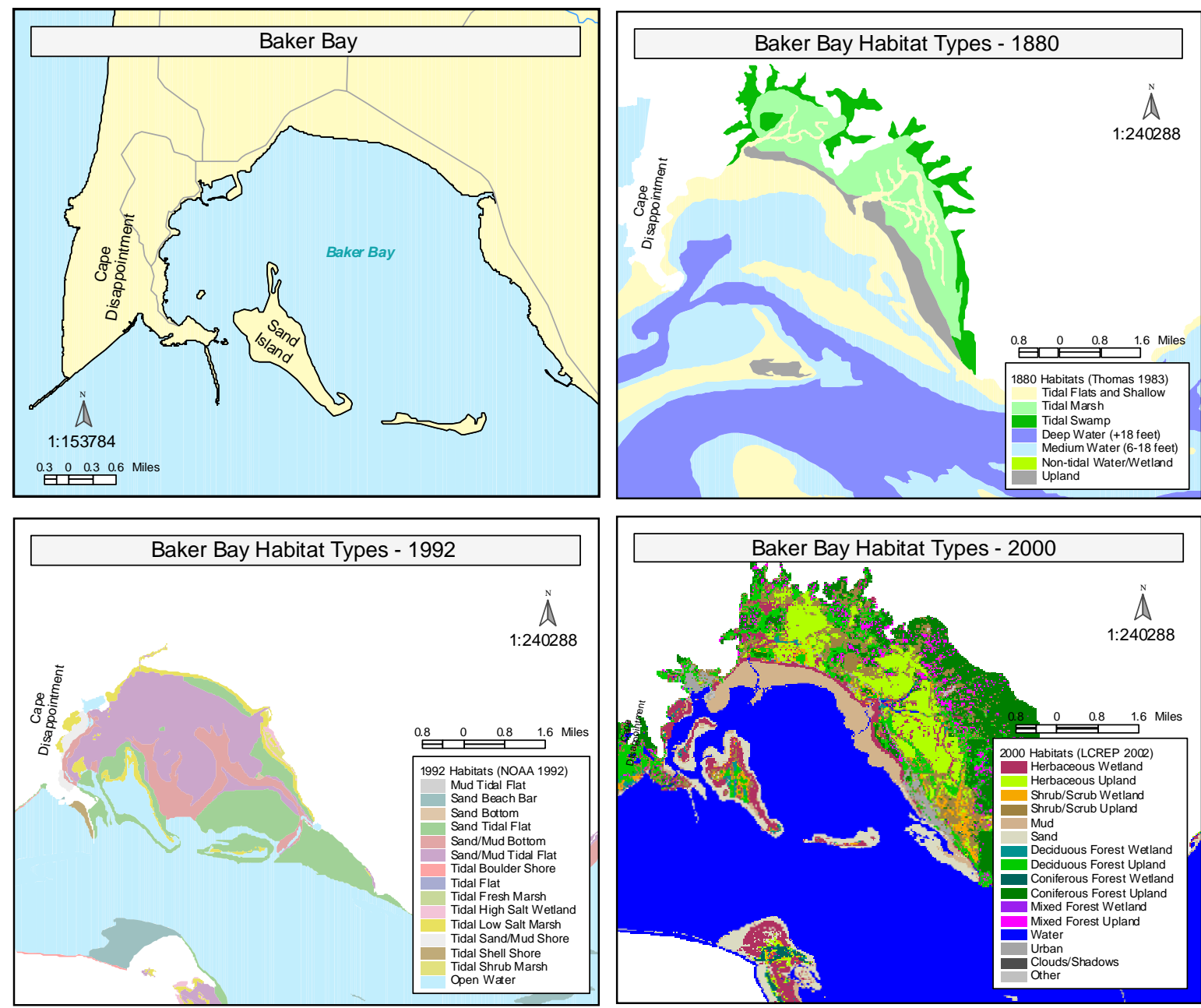

Figure 2.10. Habitat Maps of the Baker Bay Subarea

\subsubsection{Grays Bay Subarea}

As with other bays of the CRE, Grays Bay has undergone significant change in the last 100 years (Table 2.12; Figure 2.11). A significant amount of shoaling has occurred as a result of the construction of pile dikes adjacent to the main navigation channel. As a result, circulation in the area has decreased causing flooding problems for valley bottom areas in Grays River and Deep River. Dike construction has also isolated the channel from its historic flood plain. Tidal marsh has developed in the accreting bay, which has led to a net increase of

Table 2.12. Habitat Changes- Grays Bay Subarea

\begin{tabular}{cccc}
\hline Habitat Type & 1870 Acreage & 1983 Acreage & Change \\
\hline Deep Water & 2,270 & 1,690 & $-25.6 \%$ \\
Medium Depth & 2,230 & 2,040 & $-8.5 \%$ \\
Shallow/flats & 3,790 & 4,330 & $14.2 \%$ \\
Tidal Marshes & 310 & 760 & $145.2 \%$ \\
Tidal Swamps & 4,410 & 510 & $-88.4 \%$ \\
\hline
\end{tabular}
this habitat type over the last 100 years, while tidal swamps have been lost primarily due to pasture conversion activities in the lowlands. Remnant undiked areas still exist and may serve as reference patches of tidal swamp habitat for local restoration planning. 

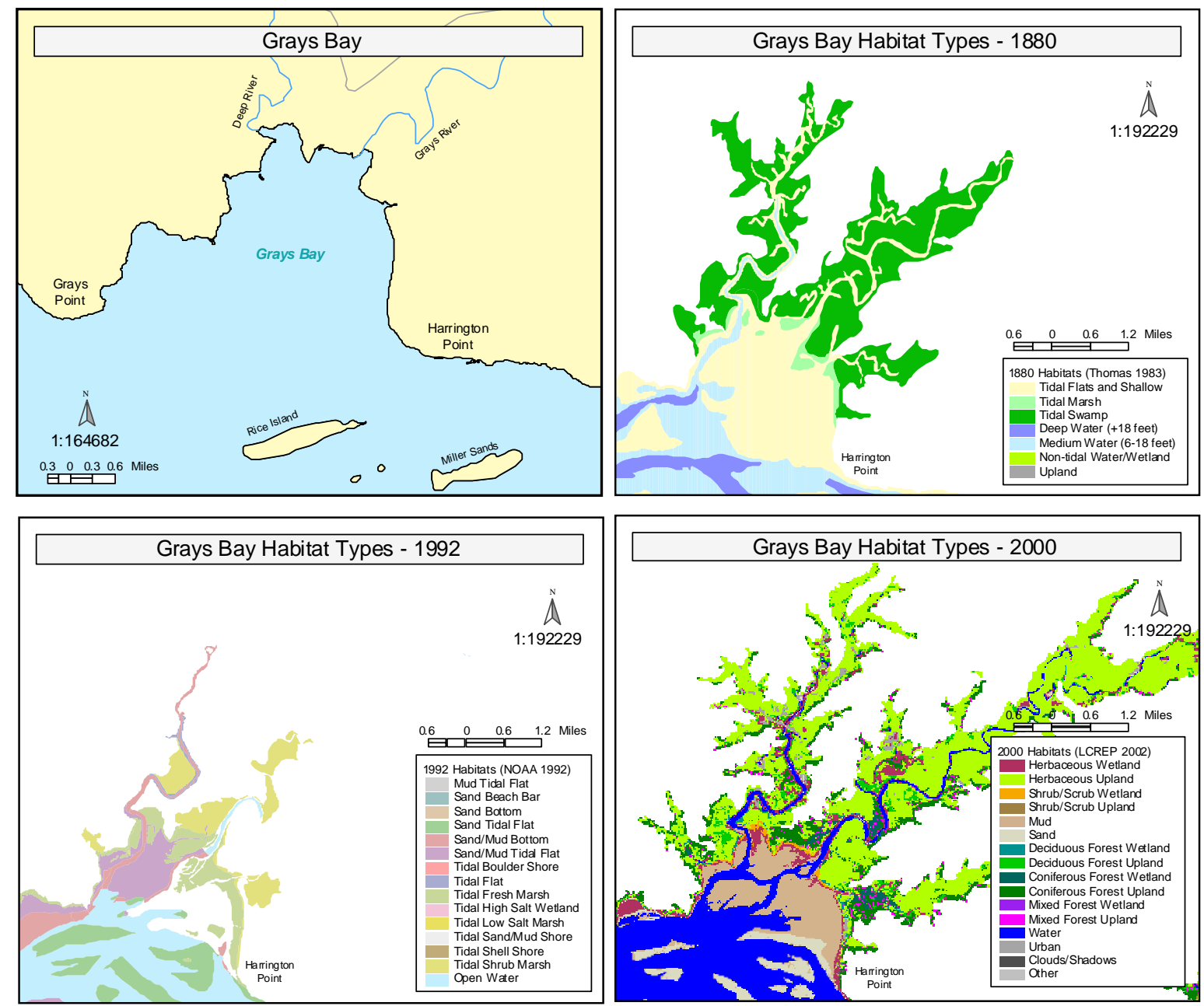

Figure 2.11. Habitat Maps of the Grays Bay Subarea

\subsubsection{Cathlamet Bay Subarea}

This subarea is home to some of the most intact, productive tidal marsh and tidal swamp in the CRE (Table 2.13; Figure 2.12). The western edge of Cathlamet Bay also encompasses a part of the brackish oligohaline zone, an area identified as an important transition area for migrating juvenile anadromous fish. A large expanse of the subarea is protected by the Lewis and Clark Refuge. Russian, Marsh, and Horseshoe Islands are all examples of pristine habitat areas of the estuary. A significant portion of this area, however, has been heavily impacted by diking, particularly the Brownsmead area and Swenson Island, contributing to the losses reported in Table 2.13. Other areas of Cathlamet Bay have been impacted by dredge material disposal as evidenced by Lois, Mott, and Millar-Pillar islands. Fridges of these areas, however, are contributing to an overall percentage gain in tidal marsh habitat (Table 2.13).
Table 2.13. Habitat Changes- Cathlamet Bay Subarea

\begin{tabular}{cccc}
\hline Habitat Type & 1870 Acreage & \multicolumn{3}{c}{1983 Acreage } & Change \\
\hline Deep Water & 6,390 & 5,590 & $-12.5 \%$ \\
Medium Depth & 8,190 & 5,700 & $-30.4 \%$ \\
Shallow/flats & 13,330 & 14,250 & $6.9 \%$ \\
Tidal Marshes & 5,580 & 5,960 & $6.8 \%$ \\
Tidal Swamps & 7,950 & 4,060 & $-48.9 \%$ \\
\hline
\end{tabular}



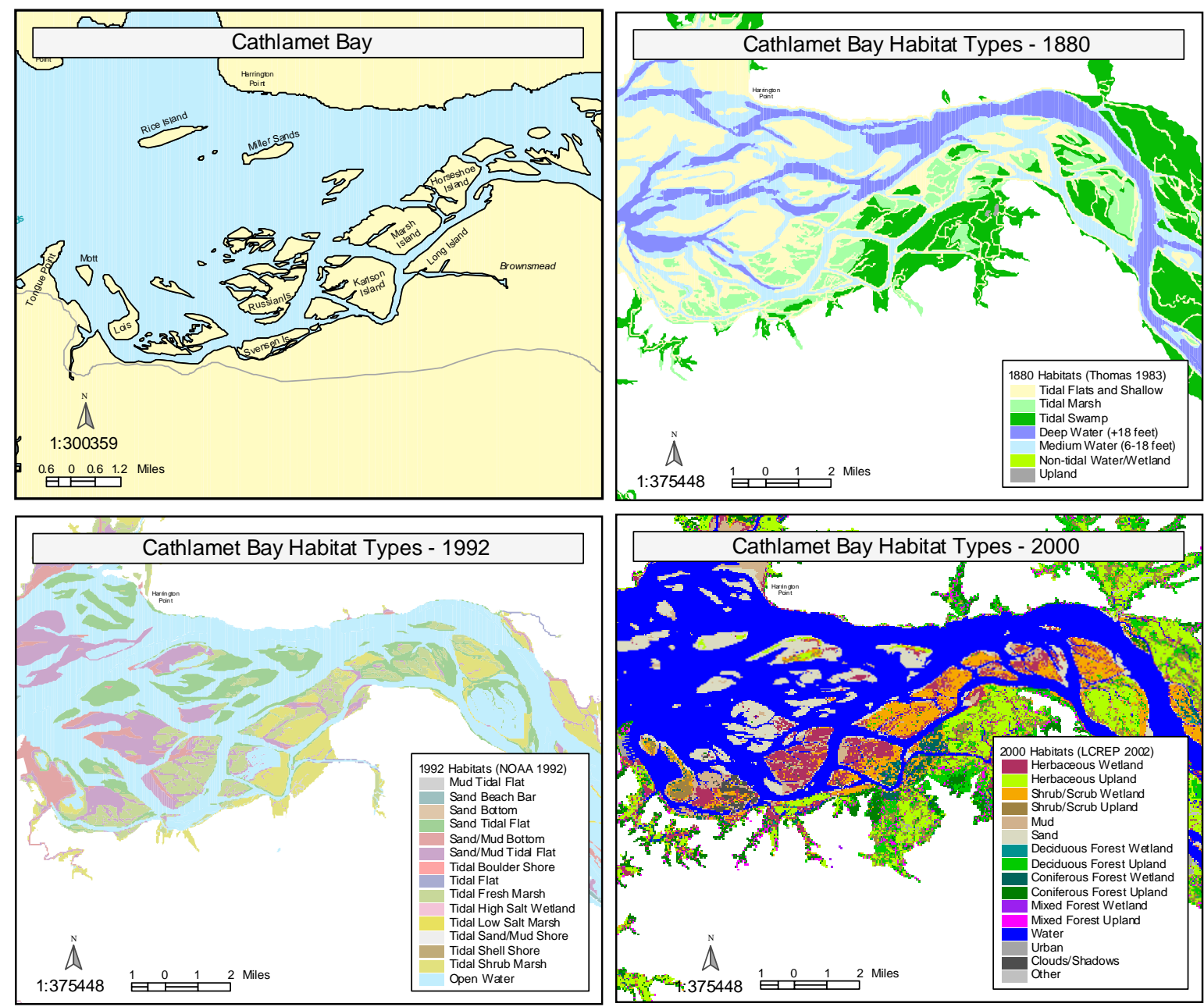

Figure 2.12. Habitat Maps of the Cathlamet Bay Subarea

\subsubsection{Upper Estuary Subarea}

The upper estuary is distinguished from the other subareas by its deep channels and steep shorelines on both the Oregon and Washington shores. The narrower channel contributes to a habitat structure dominated by more tidal swamp habitat and less edge habitat in the form of tidal marshes. This subarea is characteristically freshwater with infrequent incursions of seawater during low river flows and strong flood tides. On the Washington side, there is an extensive

Table 2.14. Habitat Changes Upper Estuary Subarea

\begin{tabular}{cccc}
\hline Habitat Type & 1870 Acreage & 1983 Acreage & Change \\
\hline Deep Water & 6,520 & 5,060 & $-22.4 \%$ \\
Medium Depth & 2,710 & 2,790 & $+3.0 \%$ \\
Shallow/flats & 1,770 & 2,710 & $+53.1 \%$ \\
Tidal Marshes & 1,430 & 510 & $-64.3 \%$ \\
Tidal Swamps & 11,180 & 2,250 & $-79.9 \%$ \\
\hline
\end{tabular}
floodplain between Cathlamet and Skamokawa. Historic tidal marsh habitat loss is most evident on areas like Tenasillahe Island. Tidal swamp loss is severe due to extensive diking and clearing on Puget Island and on the floodplains of Skamokawa and Elochohman tributary streams (Table 2.14; Figure 2.13). 

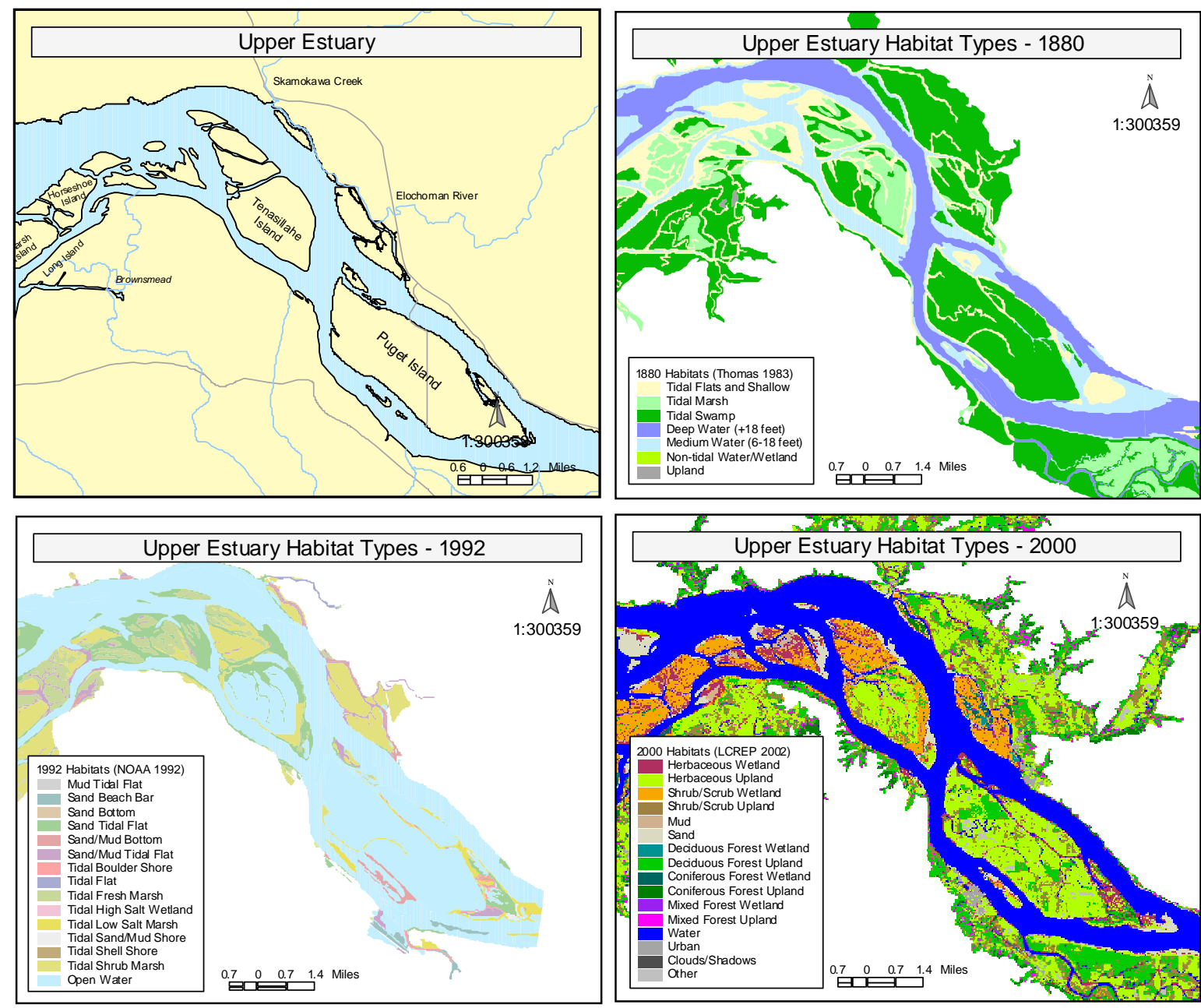

Figure 2.13. Habitat Maps of the Upper Estuary Subarea

\subsubsection{Tidal Freshwater Subsystem (RM 46-146)}

The Tidal Freshwater Subsystem (Figures 2.14 and 2.15) is noticeably distinct from the Estuarine Subsystem in its geology, vegetation, and climate. The tidal freshwater area of the CRE is influenced in part by major tributaries like the Cowlitz, Lewis, Kalama, and Willamette rivers. It has elongated islands that divide the river and form a complex of floodplain water bodies including oxbow lakes, sloughs and side channels in areas like Scappoose Bay and Sauvie Island. Historically, this subsystem was dominated by a mosaic of tidal plant communities, ash-riparian forests, and marshy lowlands. 

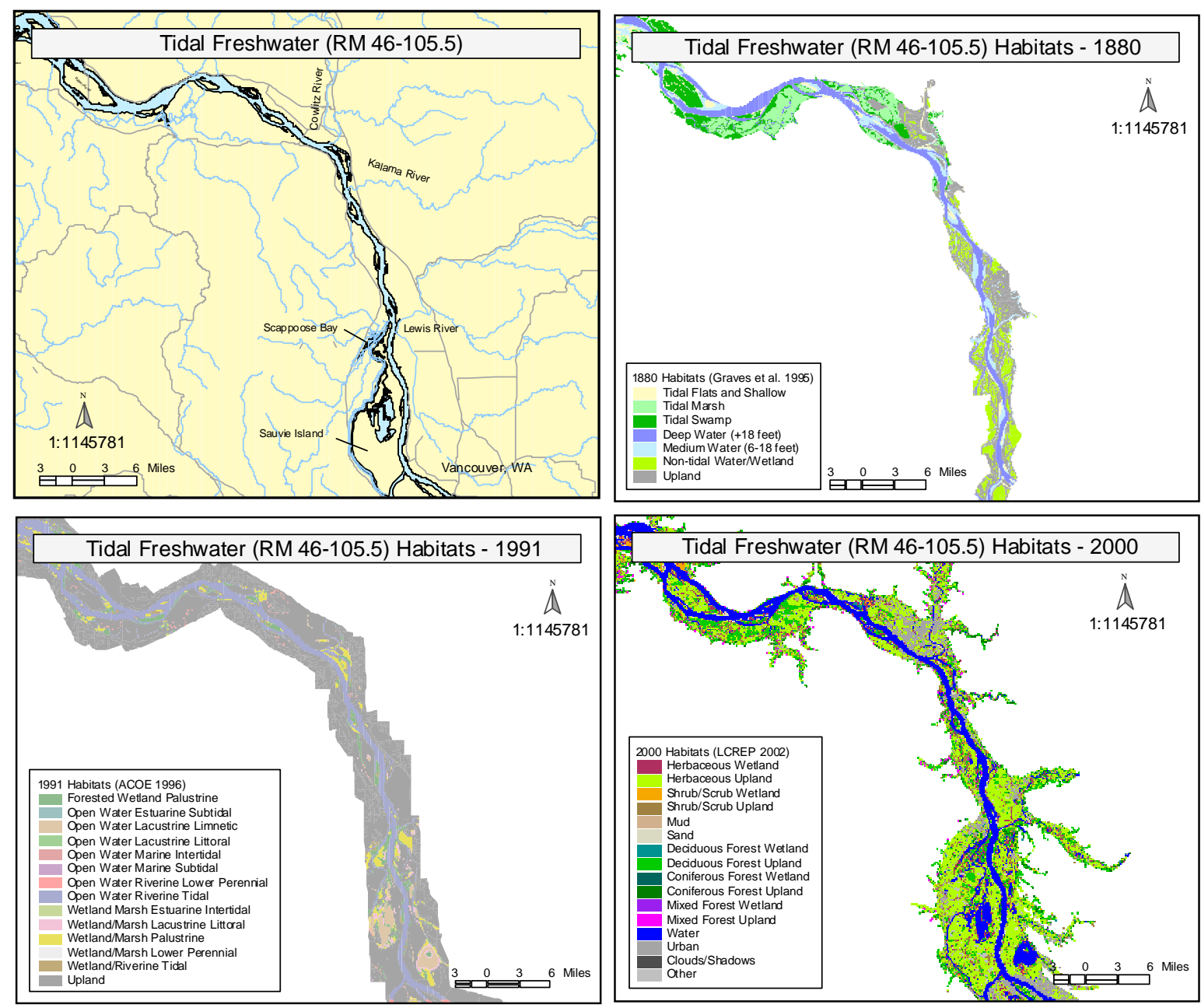

Figure 2.14. Habitat Maps of the Tidal Freshwater Subsystem (RM 46-105)

There is a comparative lack of historical data for the Tidal Freshwater Subsystem of the CRE. For example, in the tidal freshwater region of the CRE ( RM 46-146) (Figure 2.15), habitat classification work has been less extensive than in lower reaches. In RM 46-102, Graves et al. (1995) extended the habitat classification work of Thomas (1983) to provide additional historical data using survey charts from circa 1880. Little historical data interpretation exists further upriver than RM 102, besides a COE (1996) study that delineated habitat types using aerial photographs from 1948, 1961, 1973, 1983, and 1991. Graves et al. (1995) data are also available in GIS form and can be used for comparisons in the middle section only (RM 46-105.5). Comparisons could be done upriver from this using the Estuary Partnership 2000 data (Garano et al. 2002) and General Land Office (GLO) data, but are not planned at this time. The 1996 COE study also uses the Graves et al. (1995) 1880 data for comparisons within the available region of overlap. 

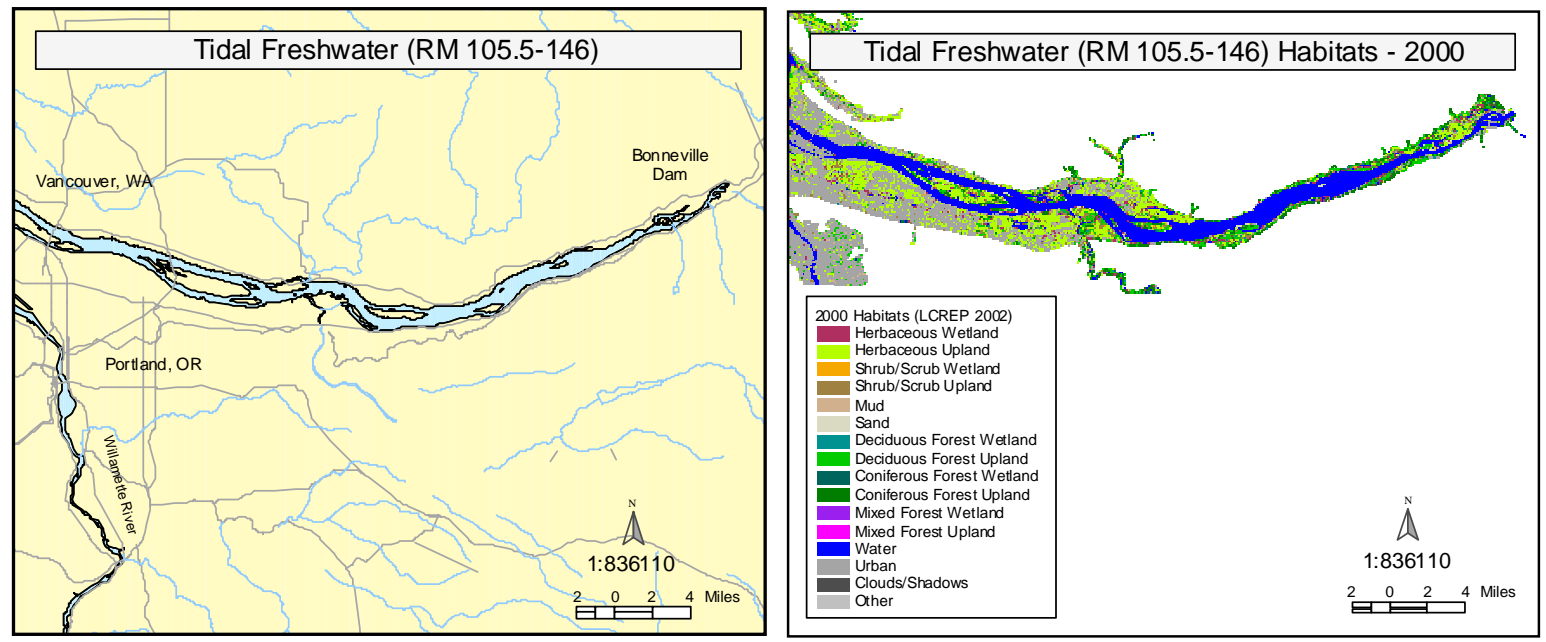

Figure 2.15. Habitat Maps of the Tidal Freshwater Subsystem (RM 105-146)

Comparing the COE 1991 data and the 1880 data in Graves et al. (1995), the following qualitative changes can be seen between RM 46 and 102 when using only the area of overlap and attempting to sort the COE-defined habitats into the Thomas/Graves types: 1) there have been extensive gains of upland habitat types in the middle reach; 2) Conversely, there appear to be extensive losses of the non-tidal water/wetland, tidal flats and shallow water, and tidal marsh habitat types; and (3) the COE classification did not include a category that fit well with the tidal swamp type, so no current data are available for comparison.

For the upper reach section (RM 105.5-146; Figure 2.15), it is necessary to rely on the analysis done by the COE comparing various aerial photographs between 1948 and 1991. In general, the following changes can be observed from 1948 to 1991 (again sorting into the Thomas classes): 1) there have been gains of the non-tidal water/wetland and upland habitats in the upper reach; 2) there have been some losses in tidal flats and shallows; 3 ) there has been a significant reduction in tidal marsh; and 4) again, tidal swamp data are not available.

The following difficulties arose when we compared the current and historical habitat maps:

- non-overlapping coverage areas

- lack of spatial resolution of historical datasets - Some comparisons can be made, but detailed analysis cannot be done. The low resolution also prevents complete characterization of important habitats, as many of the habitats may be below the detectable size

- different classification schemes - the COE attempted to resolve this by lumping categories into a few "like" types for the comparisons, though this is fundamentally difficult due to the classifications originally used.

Thus, the available datasets allow comparison of only the total acreage of habitat types. Landscape position, patch size, and other factors important to salmonid use cannot be analyzed at the scales currently available. However, the ongoing projects by NOAA Fisheries and the Estuary Partnership will provide the kind of detail to address these factors at key focal areas within the CRE. 


\subsubsection{Summary of Habitat Changes}

The information on habitat loss for the area from the river entrance to RM46 is shown in Figure 2.16. Historical data for the region above RM46 have only been analyzed for small portions of the study area. Development along the shoreline especially in Astoria and other smaller communities along the system has modified shoreline conditions. Tidal swamps have suffered the greatest loss, followed by tidal marshes and medium-depth areas. Shallows and flats have increased slightly in area, and deep areas have decreased slightly in area.

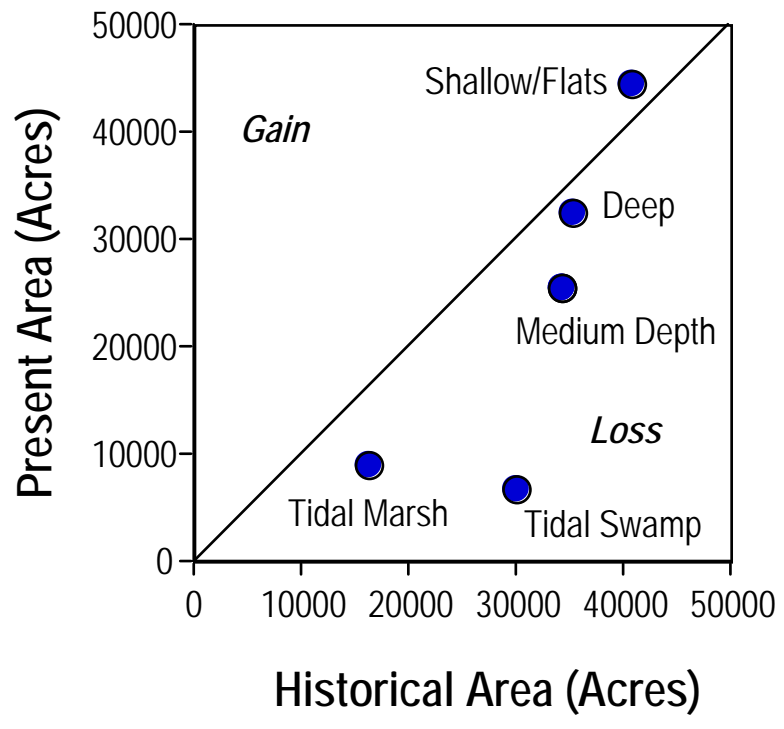

Figure 2.16. Changes in Area Covered by Aquatic Habitat Types in the Estuarine Subsystem (RM 0-46). Habitat types are defined in Section 2.4.1. (Analogous data for RM 46-146 are not available.)

Figure 2.17 illustrates the habitat losses by subarea for each habitat type. The Entrance Subarea has seen a moderate decline in shallows and flats and medium-depth habitats and a slight increase in deep habitat. Deep habitat was historically the most prominent habitat type in the Entrance Subarea. There has been little change in the prominent historical habitats (i.e., shallows and flats, medium depth, deep) in the mixing zone. Baker Bay has undergone large losses of tidal swamp, tidal marsh, medium depth, and deep habitats and has shown a large increase in shallows and flats, the historically dominant habitat type in that subarea. There has been a major decline in the formerly dominant tidal marshes in the Youngs Bay Subarea. Tidal swamps have also been essentially eliminated from Youngs Bay. Other habitat types remain similar to historical conditions. The Grays Bay subarea has also seen a major loss of tidal swamp, which was the co-dominant historical habitat along with shallows and flats. There has been a slight increase in tidal marsh area in Grays Bay and little change in other habitat types. Cathlamet Bay has lost some of its deep and medium-depth habitat, but has seen a slight increase in shallows and flats and tidal marshes. Tidal swamps, a prominent habitat historically, have had moderate losses in Cathlamet Bay. The Upper Estuary Subarea has seen the largest loss of tidal swamps among all subareas. This subarea has also lost tidal marshes. 

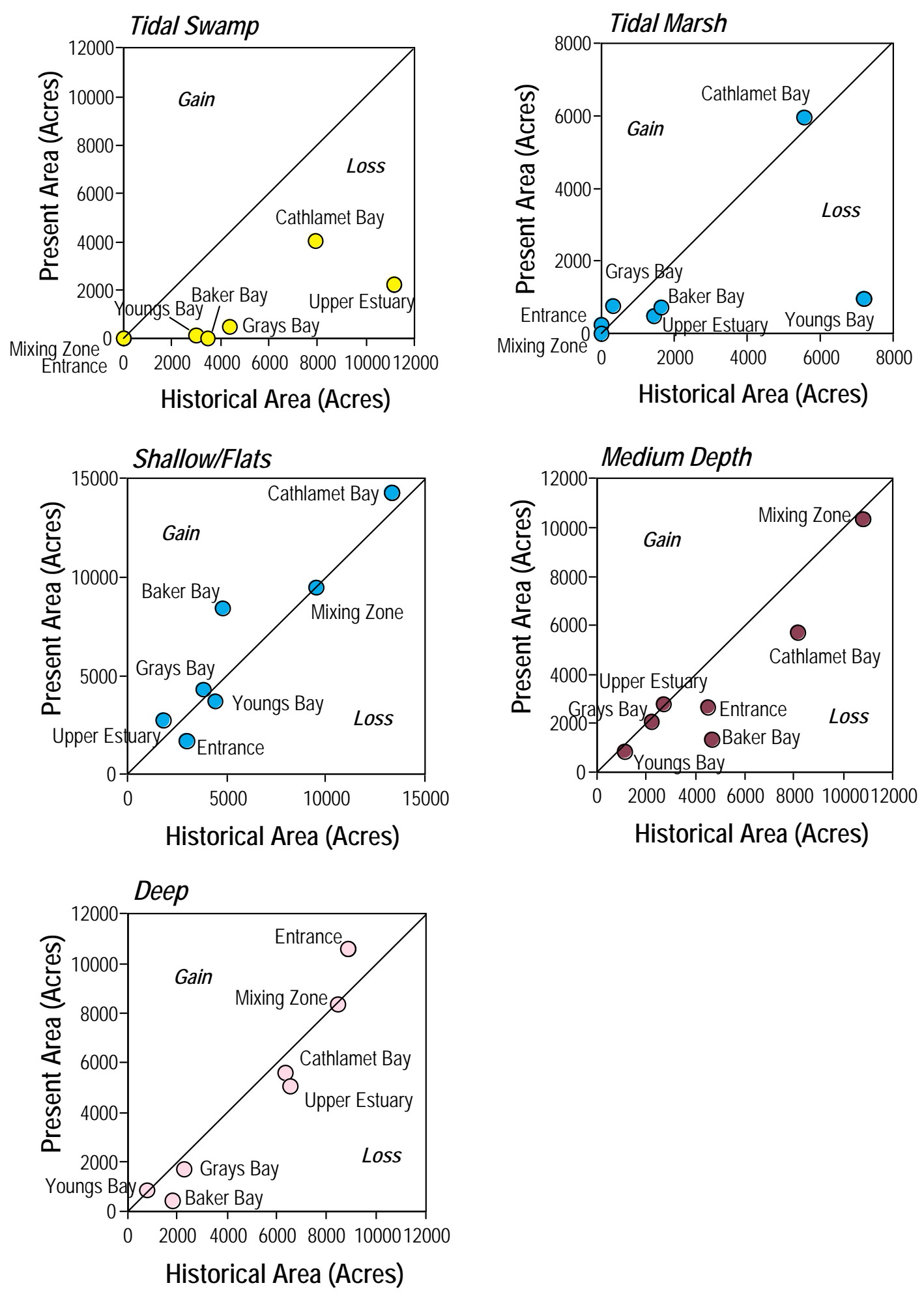

Figure 2.17. Historical and Present-Day Coverage of Five Habitat Types within the Seven Subareas of the Estuarine Subsystem (RM 0-46). Habitat types are defined in Section 2.4.1. 
Tidal swamp and tidal marsh habitats have been reduced the most (74\% and 49\%, respectively) since 1870 according to Thomas' (1983) assessment (Table 2.15). Shallow/flat habitat has increased by 10\%, in part because of dredge-material placement and residual shoaling. Figures 2.16 and 2.17 provide a direction and relative need for restoration of the various habitat types within each subarea. The massive loss of tidal swamp habitat in the upper estuary indicates that, to address the general goal for restoration in the CRE, considerable effort should be placed on restoring tidal swamp and tidal marsh habitats. Similarly, in Youngs Bay, restoration should focus primarily on tidal marsh restoration. These recommendations assume that it will be worthwhile ecologically to regain lost habitat types; research is underway to address this assumption. Future studies on habitat conditions should correspond directly to the existing data sets developed by Thomas (1983) and forthcoming sets of historical data to be developed by NOAA Fisheries.

In summary, historical changes in the system have primarily involved changes related to flow regulation and dredging, diking and filling. These alterations have resulted in altered hydrodynamics, bathymetry, and water properties (e.g., salinity range, nutrient input). Flow regulation has changed the range and location of the estuarine turbidity maximum, nutrient processing, sedimentation, elevation, current velocities and flushing of the system. Diking and filling have altered tidal exchanges of materials and water in tidal swamps and marshes. Bathymetry changes have essentially resulted in alterations of the amounts and distribution of benthic, soft bottom habitat types.

Table 2.15. Summary Totals for the Habitat Changes Presented in Tables $2.8-2.14$ for the Estuarine Subsystem.

\begin{tabular}{cccc}
\hline Habitat Type & $\mathbf{1 8 7 0}$ & $\mathbf{1 9 8 3}$ & \% change \\
\hline Deep Water & 36,820 & 32,130 & -13 \\
Medium Depth & 31,150 & 25,100 & -19 \\
Shallow/flats & 40,640 & 44,770 & +10 \\
Tidal Marshes & 19,240 & 9,820 & -49 \\
Tidal Swamps & 28,340 & 7,400 & -74 \\
\hline
\end{tabular}

\subsection{Restoration Strategies for the CRE}

To accomplish the goal (Section 1.2) of implementing scientifically sound projects to restore tidal wetlands and other presumably key habitats, the controlling factors relevant to these habitats must be either in place or re-established at the levels appropriate to support habitat structure and function. Because of changes in hydrological conditions caused by flow regulation, coupled with loss of tidal wetlands, the system in the CRE has been altered significantly from historical conditions (Section 2.4). Evaluated in terms of the restoration success framework (Figure 2.3), there have been major changes at both the landscape scale and the site scale (NRC 1992; Shreffler and Thom 1993). While historical conditions may be assumed to represent an optimal habitat conditions for a particular site, it must be noted that present-day conditions of the CRE may constrain the type, size, and functionality of said habitat conditions. Hence, optimal habitat conditions for a site under present-day CRE conditions may differ from optimal historical conditions. In any event, a return to a more "normative" state than is currently present may be advantageous (Independent Scientific Group 2000). 
As discussed in Section 2.1.3, there are several potential pathways for "restoration," which range from restoring the system to historical conditions to creating new habitat. In general, restoration to historical conditions is the best strategy if the site and the landscape are not heavily altered (NRC 1992). Creation of new habitat (i.e., habitat not historically present) at a site is best applied when the site and the landscape are heavily damaged. Because parts of the CRE have been heavily altered, and some of these alterations will persist into the future, the goal of restoring the habitats to historical conditions may not be viable over these parts of the system. In general, though, we recommend a strategy that seeks, where practical, to increase the amount of habitat that has been depleted, such as tidal marsh and swamp.

All potential strategies should be evaluated at a potential restoration site. Where restoration to historical conditions appears to be possible, then this should be strongly considered as the most beneficial option. In addition, pockets of habitat exist in the system that appear to be highly functional (e.g., tidal marshes and channels in Cathlamet Bay; the flats in Baker Bay). In areas such as these, the additional strategies of conservation and protection should be considered. The following material outlines a process for prioritizing strategies for various sites in the CRE. The objective here is to develop science-based protocol for determining priorities and strategies to improve ecosystem functions in the CRE. This process links output from the conceptual model, the description of juvenile salmon usage, and the assessment of existing habitat conditions (Sections 2.2,2.3, and 2.4, respectively) with the fundamental strategies for restoration (Section 2.1) to drive the prioritization process. The process draws from the fields of restoration ecology, landscape ecology, and conservation biology. The material here focuses on finding the most useful restoration strategies. Later in the document (Section 3.2), the focus is on a process to prioritize restoration projects.

As developed in Section 2.1, landscape- and site-specific disturbance levels are key factors to consider when selecting sites and strategies. Loss and alteration of aquatic habitats (Section 2.4) has been the major change in the CRE system besides flow regulation. The matrix in Figure 2.18 identifies the strategies most appropriate for the nine states of reach and management unit disturbance. In addition, the matrix includes a "strategy" of restricted development. This refers to locations where disturbance is already moderate to high, as in heavily altered or disturbed, and development using established environmental regulations is followed. Restricted development should use other strategies to avoid and minimize further disturbances following general conservation and restoration ecology principles.

As seen in the matrix (Figure 2.18), multiple strategies are potentially viable for any one of the nine states. This matrix provides general guidance as a first approximation of specific management actions that could be evaluated within a reach or management unit. In developing the matrix in Figure 2.18, the following logic was used:

- the lower the disturbance on both scales, the greater the reliance on protection, conservation, and restoration.

- the greater the disturbance on both scales, the greater the reliance on enhancement

- under the greatest levels of disturbance, the greater the reliance on creation with more restricted development allowed. 


\begin{tabular}{|c|c|c|c|}
\hline $\begin{array}{c}\text { Subarea Disturbance } \\
\text { High }\end{array}$ & $\begin{array}{c}\text { Create } \\
\text { Enhance } \\
\text { Restore }\end{array}$ & $\begin{array}{c}\text { Create } \\
\text { Enhance } \\
\text { Restore } \\
\text { Restricted Devel. }\end{array}$ & $\begin{array}{c}\text { Create } \\
\text { Enhance } \\
\text { Restricted Devel. }\end{array}$ \\
\hline $\begin{array}{c}\text { Subarea Disturbance } \\
\text { Moderate }\end{array}$ & $\begin{array}{c}\text { Enhance } \\
\text { Restore } \\
\text { Protect }\end{array}$ & $\begin{array}{l}\text { Conserve } \\
\text { Create } \\
\text { Enhance } \\
\text { Restore }\end{array}$ & $\begin{array}{c}\text { Conserve } \\
\text { Enhance } \\
\text { Restore } \\
\text { Restricted Devel. }\end{array}$ \\
\hline \multirow[t]{2}{*}{$\begin{array}{c}\text { Subarea Disturbance } \\
\text { Low }\end{array}$} & $\begin{array}{c}\text { Conserve } \\
\text { Restore } \\
\text { Protect }\end{array}$ & $\begin{array}{c}\text { Conserve } \\
\text { Enhance } \\
\text { Restore }\end{array}$ & Enhance \\
\hline & $\begin{array}{c}\text { Subsystem } \\
\text { Disturbance } \\
\text { Low }\end{array}$ & $\begin{array}{c}\text { Subsystem } \\
\text { Disturbance } \\
\text { Moderate }\end{array}$ & $\begin{array}{c}\text { Subsystem } \\
\text { Disturbance } \\
\text { High }\end{array}$ \\
\hline
\end{tabular}

Figure 2.18. Matrix of habitat restoration strategies appropriate for increasing degrees of disturbance by subsystem and subarea. The restricted development strategy is described in Section 2.14; the other strategies are described in Section 2.1.3. Generally, the probability of success increases as the subarea and subsystem disturbance levels decrease (see Figure 2.3, where local and landscape are analogous to subarea and subsystem, respectively).

For restoration to be successful in the CRE, natural processes that form and maintain habitats need to be intact in the landscape where these subareas reside. In the present case, the subsystems (zones) as defined by CREDDP (Thomas 1983; Figure 1.1) can be considered to be the "landscape scale," and disturbances on this scale will affect the probability of success. A national assessment showed that the degree of disturbance on the landscape (subsystem) and site (areas within a subarea) scales affected the probability of restoration success and that the most appropriate restoration strategies varied according to disturbance on these two scales (Figure 2.3) (NRC 1992; Thom 2000).

To help refine the restoration strategies most applicable to the habitats in the CRE, the types of disturbances that have taken place were related to effects on key controlling factors. The factors controlling habitat type are shown in the habitat forming submodel in Figure 2.4. Indicators of the consequences and degree of disturbance in hydrodynamics, bathymetry, and salinity are shown in Table 2.16. Because apparent alterations in habitat structure and function have not been fully studied, we relied on indicators of the level of disturbance of the system. The metrics provide a direct indication of the impact on the habitats and biota, and information on the metrics is readily available.

The metrics provide an indirect means to assess the relative value of restoring the controlling factors that will lead to a restored ecosystem. Diking and filling have been a primary cause of decreases in tidal swamp and marsh area in the CRE (see Section 2.4). These actions effectively eliminated the exchange of water, materials and organisms between the larger aquatic system and the adjacent, shallowly vegetated habitats, although some exchange occurs through tide gates. Exchange is important not only for the access and use of these habitats by fish, but also for maintenance of habitat-forming processes such as sedimentation and erosion. By restoring tidal exchange, these processes are re-established, and the habitats can develop naturally as well as provide their functions to the ecosystem. For example, if there 
has been a very large change in the bathymetry of a subarea, restoration of the bathymetry would be the action best suited for restoring or improving benthic biota and habitats. Other actions may also be effective in a particular subarea. The assessment of the disturbance level with each CRE subarea for the three controlling factors is shown in Table 2.17.

Table 2.16. Associated Alterations and Metrics Indicating the Degree of Disturbance for Three Controlling Factors in the CRE

\begin{tabular}{lll}
\hline $\begin{array}{l}\text { Controlling } \\
\text { Factor }\end{array}$ & Associated Alteration & $\begin{array}{l}\text { Metric Indicating Degree of Disturbance } \\
\text { Used Here }\end{array}$ \\
\hline Hydrodynamics & $\begin{array}{l}\text { Current velocities } \\
\text { ETM location and dynamics } \\
\text { Salinity locations and dynamics } \\
\text { Tidal exchange to marshes and swamps }\end{array}$ & Change in tidal marsh and swamp area \\
& $\begin{array}{l}\text { Deposition zones } \\
\text { Sediment loads }\end{array}$ & \\
& $\begin{array}{l}\text { Plankton dynamics and composition } \\
\text { Benthic habitat type }\end{array}$ & $\begin{array}{l}\text { Change in area of shallows/flats, medium } \\
\text { depth and deep areas }\end{array}$ \\
& $\begin{array}{l}\text { Juvenile salmon habitat } \\
\text { Currents and water property dynamics }\end{array}$ & \\
Salinity & $\begin{array}{l}\text { Benthic and planktonic biota composition, } \\
\text { distribution and dynamics }\end{array}$ & $\begin{array}{l}\text { Change in historical versus present range of } \\
\text { salinity intrusion }\end{array}$ \\
\hline
\end{tabular}

The matrix of habitat action strategies in Figure 2.19 can be used to prioritize appropriate habitat restoration strategies within these zones and subareas. Bathymetry represents a more landscape-scale factor and tidal exchange represents a site-scale factor but both bathymetry and tidal exchange exert strong controls on habitat formation. Salinity, although important, was not included in Figure 2.19 because salinity co-varies partially with bathymetry and tidal exchange. For projects in subareas with low disturbance on both scales, the most appropriate habitat action strategies would be conserve, protect, and restore (to pre-disturbance or pre-historical conditions) (Figure 2.19). For projects where disturbance is high on both scales, habitat action strategies of enhancement of selected habitat attributes, creation of new ecosystems or restricted development are most appropriate. However, any strategy for restoration needs to be considered relative to the reduced ability of processes disturbed landscape to maintain a restored site in the long term. 
Table 2.17. Summary of Controlling Factor Disturbance Level within each Subarea of the Estuarine and Tidal Freshwater Subsystems (zones). Levels of disturbance are low $(L)$, medium $(M)$, and high $(H)$. For a more complete description, see Section 2.5 text.

\begin{tabular}{|c|c|c|c|c|}
\hline $\begin{array}{l}\text { Subsystem } \\
\text { (zone) }\end{array}$ & Subarea & Tidal Exchange & Bathymetry & Salinity \\
\hline \multirow[t]{7}{*}{ Estuarine } & Entrance & $\begin{array}{l}\text { L-only a small area } \\
\text { of historical marshes } \\
\text { and swamps }\end{array}$ & $\begin{array}{l}\text { H-very large } \\
\text { increases in deep } \\
\text { water area, and loss } \\
\text { of medium and } \\
\text { shallow depth areas }\end{array}$ & $\begin{array}{l}\text { L-probably } \\
\text { somewhat less } \\
\text { dynamic, but still } \\
\text { ocean-dominated }\end{array}$ \\
\hline & Mixing Zone & $\begin{array}{l}\text { L-only a small area } \\
\text { of historical marshes } \\
\text { and swamps }\end{array}$ & $\begin{array}{l}\text { L-little change in } \\
\text { area, although high } \\
\text { degree of shifting of } \\
\text { locations }\end{array}$ & $\begin{array}{l}\text { M-very dynamic } \\
\text { salinity zone, } \\
\text { probably altered by } \\
\text { flow regulation }\end{array}$ \\
\hline & Youngs Bay & $\begin{array}{l}\text { H-substantial loss of } \\
\text { tidal marsh and } \\
\text { swamp }\end{array}$ & $\begin{array}{l}\text { M-loss of medium } \\
\text { and shallow depth } \\
\text { areas }\end{array}$ & $\begin{array}{l}\text { M-very dynamic } \\
\text { salinity zone, } \\
\text { probably altered by } \\
\text { flow regulation }\end{array}$ \\
\hline & Baker Bay & $\begin{array}{l}\text { H-substantial loss of } \\
\text { tidal marsh and } \\
\text { swamp }\end{array}$ & $\begin{array}{l}\text { H-substantial loss of } \\
\text { deep and medium } \\
\text { deep areas, and } \\
\text { increase in shallow } \\
\text { areas }\end{array}$ & $\begin{array}{l}\text { M-very dynamic } \\
\text { salinity zone, } \\
\text { probably altered by } \\
\text { flow regulation }\end{array}$ \\
\hline & Grays Bay & $\begin{array}{l}\text { H-substantial loss of } \\
\text { tidal swamp }\end{array}$ & $\begin{array}{l}\text { M-shift from } \\
\text { deepwater area to } \\
\text { shallow flats }\end{array}$ & $\begin{array}{l}\text { L-a small change in } \\
\text { dilute salinity } \\
\text { dynamics }\end{array}$ \\
\hline & $\begin{array}{l}\text { Cathlamet } \\
\text { Bay }\end{array}$ & $\begin{array}{l}\text { M-loss of tidal } \\
\text { swamps, but gain in } \\
\text { tidal wetlands }\end{array}$ & $\begin{array}{l}\text { M-loss of deep and } \\
\text { medium deep areas }\end{array}$ & $\begin{array}{l}\text { L-a small change in } \\
\text { dilute salinity } \\
\text { dynamics }\end{array}$ \\
\hline & $\begin{array}{l}\text { Upper } \\
\text { Estuary }\end{array}$ & $\begin{array}{l}\text { H-substantial loss of } \\
\text { tidal swamp and } \\
\text { marsh }\end{array}$ & $\begin{array}{l}\text { H-loss of deep and } \\
\text { gain in medium deep } \\
\text { area, and substantial } \\
\text { increase in shallow } \\
\text { areas }\end{array}$ & $\begin{array}{l}\text { L-a small change in } \\
\text { dilute salinity } \\
\text { dynamics }\end{array}$ \\
\hline \multirow[t]{2}{*}{$\begin{array}{l}\text { Tidal } \\
\text { Freshwater }\end{array}$} & $\begin{array}{l}\text { Middle Reach } \\
\text { (RM46-102) }\end{array}$ & $\begin{array}{l}\text { H-substantial loss of } \\
\text { tidal swamp and } \\
\text { marsh, and non-tidal } \\
\text { wetland }\end{array}$ & $\begin{array}{l}\text { H-loss of shallow } \\
\text { area, and gain in deep } \\
\text { area }\end{array}$ & L-salinity not a factor \\
\hline & $\begin{array}{l}\text { Upper Reach } \\
\text { (RM 102- } \\
146)\end{array}$ & $\begin{array}{l}\text { H-substantial loss of } \\
\text { tidal swamp and } \\
\text { marsh suspected, and } \\
\text { gain in non-tidal } \\
\text { wetland }\end{array}$ & $\begin{array}{l}\text { H-loss of shallow } \\
\text { area, and gain in deep } \\
\text { area }\end{array}$ & L-salinity not a factor \\
\hline
\end{tabular}




\begin{tabular}{c|c|c|c|}
\hline $\begin{array}{c}\text { Tidal Exchange } \\
\text { Disturbance } \\
\text { High }\end{array}$ & & $\begin{array}{c}\text { Youngs Bay } \\
\text { Grays Bay }\end{array}$ & $\begin{array}{c}\text { Baker Bay } \\
\text { Upper Estuary } \\
\text { Tidal Freshwater }\end{array}$ \\
\hline $\begin{array}{c}\text { Tidal Exchange } \\
\text { Disturbance } \\
\text { Moderate }\end{array}$ & Cathlamet Bay & \\
\hline $\begin{array}{c}\text { Tidal Exchange } \\
\text { Disturbance } \\
\text { Low }\end{array}$ & Mixing Zone & & Entrance \\
\hline & $\begin{array}{c}\text { Bathymetry } \\
\text { Disturbance } \\
\text { Low }\end{array}$ & $\begin{array}{c}\text { Bathymetry } \\
\text { Disturbance } \\
\text { Moderate }\end{array}$ & $\begin{array}{c}\text { Bathymetry } \\
\text { Disturbance } \\
\text { High }\end{array}$ \\
\hline & & M &
\end{tabular}

Figure 2.19. The estuary subareas and the tidal freshwater subsystem relative to disturbances in tidal exchange and bathymetry (from Table 2.17).

\subsection{The Scientific Basis for CRE Restoration: Results of the Analysis}

Decisions on restoration projects will be necessary even with uncertainties in the scientific knowledge base about juvenile salmon usage of estuarine and tidal freshwater habitats. Combining the information in Figures 2.17 and 2.18 results in recommended restoration strategies for each subarea (Table 2.18). These recommendations should be considered as priority strategies, but should not necessarily preclude any others. For example, it would be advisable to protect a particular piece of tidal marsh in Cathlamet Bay if given the opportunity. Also, the relative lack of data in the tidal freshwater subsystem will not preclude consideration of restoration actions, which will be closely monitored, in this 100-mile stretch of the CRE. Implementation guidelines for projects using these strategies are presented below.

Table 2.18. Recommended CRE Restoration Strategies by Subarea Based on the Disturbance Matrix. Note: the lack of a check in a certain subarea does not mean a particular strategy would not necessarily work in that area. Each project must be viewed on a case-by-case basis. A final decision will require a site characterization.

\begin{tabular}{lcccccc}
\hline Subarea & Conserve & Create & Enhance & Restore & Protect & Restricted Development \\
\hline Entrance & & & $\checkmark$ & & & \\
Mixing Zone & $\checkmark$ & & & $\checkmark$ & $\checkmark$ & $\checkmark$ \\
Youngs Bay & & $\checkmark$ & $\checkmark$ & $\checkmark$ & & $\checkmark$ \\
Baker Bay & & $\checkmark$ & $\checkmark$ & & $\checkmark$ \\
Grays Bay & & $\checkmark$ & $\checkmark$ & $\checkmark$ & $\checkmark$ \\
Cathlamet Bay & $\checkmark$ & $\checkmark$ & $\checkmark$ & $\checkmark$ & $\checkmark$ \\
Upper Estuary & & $\checkmark$ & $\checkmark$ & & \\
Tidal FW & & $\checkmark$ & $\checkmark$ & & & \\
\hline
\end{tabular}




\subsection{Implementation Guidelines}

This section provides guidelines to implement ecosystem restoration projects consistent with the CRE restoration strategies recommended above (Section 2.6). A variety of initiatives and partnerships are currently in place to apply ecosystem-based strategies to improve salmonid habitat conditions. In the CRE, there are numerous federal, state, and local efforts aimed at improving aquatic ecosystem conditions; the respective roles and responsibilities are explained in Appendix A. Although some of the ecological complexities of the CRE are not well understood, on-the-ground opportunities exist to apply what is known, then learn in the process. To that end, this chapter articulates guidelines to implement an effective and coordinated approach to salmon habitat restoration in the study area. Recall, the overall goal for the habitat restoration effort is as follows (from Section 1.2):

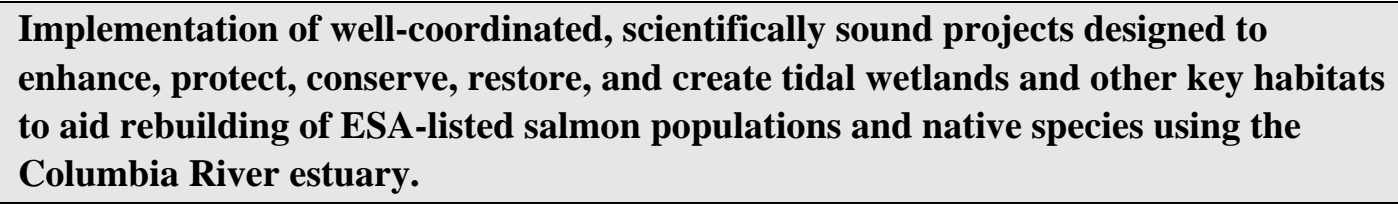

This chapter starts by describing specific types of projects that meet the restoration strategies developed above. Then project selection guidelines are proposed. These are followed by a description of the main phases of a restoration project. A process to coordinate, monitor performance, collect and disseminate data, and adaptively manage multiple projects is also described.

\subsection{Types of On-the-Ground Projects to Implement CRE Restoration Strategies}

Various types of on-the-ground projects can carry out the scientifically based restoration strategies (Table 3.1). Many of these project types are already being pursued by groups actively involved in various elements of restoration in the study area. Effectiveness monitoring is in progress and, as information becomes available, it will be incorporated into the habitat restoration planning process. For each restoration strategy (conservation, creation, enhancement, restoration, and protection), we outline the types of projects that would fit into a coordinated approach to improving ecological conditions in the CRE.

It is beyond the scope of this document to inventory diked lands and passage barriers in order to identify potential restoration sites. Marshall et al. (1987) provided an inventory of private and public diked tidelands. In addition, the BPA, the COE, and the Estuary Partnership are working together to identify restoration projects. 
Table 3.1. Examples of Project Types and Targeted Ecosystem Benefit by Restoration Strategy for the CRE.

\begin{tabular}{|c|c|c|}
\hline Strategy & Project Type & Targeted Ecosystem Benefit \\
\hline \multirow[t]{4}{*}{ Conservation } & Land conservation & $\begin{array}{l}\text { Limits land use impacts harmful to salmon habitat such as } \\
\text { sediment, contaminants, nutrient loading. }\end{array}$ \\
\hline & Easements & $\begin{array}{l}\text { Benefits ecological features through legal protection of } \\
\text { critical areas, potentially allowing for complimentary } \\
\text { restoration strategies to take place. }\end{array}$ \\
\hline & Riparian fencing & $\begin{array}{l}\text { Deters livestock activities from degrading stream side } \\
\text { areas. }\end{array}$ \\
\hline & Manure management & $\begin{array}{l}\text { Minimizes the inputs of nutrients and bacteria into stream } \\
\text { corridor. }\end{array}$ \\
\hline \multirow[t]{2}{*}{ Creation } & Material placement & $\begin{array}{l}\text { Mimics habitat function and complexity through the } \\
\text { placement of material at a given elevation. }\end{array}$ \\
\hline & Tidal channel modification & $\begin{array}{l}\text { Restores more natural flows and mimic tidal channel } \\
\text { structure. }\end{array}$ \\
\hline \multirow[t]{5}{*}{ Enhancement } & Riparian plantings & $\begin{array}{l}\text { Promotes water temperature reduction, contaminant } \\
\text { removal, connection of terrestrial habitat corridors, } \\
\text { sediment reduction, water storage, future source of large } \\
\text { woody debris input. }\end{array}$ \\
\hline & $\begin{array}{l}\text { Tide gate/culvert } \\
\text { replacement }\end{array}$ & $\begin{array}{l}\text { Promotes water temperature reduction, dissolved oxygen } \\
\text { availability, increased habitat access. }\end{array}$ \\
\hline & Invasive species removal & Increases opportunities for native species propagation. \\
\hline & $\begin{array}{l}\text { Bioengineered streambank } \\
\text { stabilization }\end{array}$ & Reduces sediment load, diffuses hydrologic energy. \\
\hline & Riparian fencing & Protects riparian zones from disturbances. \\
\hline \multirow[t]{4}{*}{ Restoration } & Tide gate removal & $\begin{array}{l}\text { Restores partial or full hydrologic connection to slough } \\
\text { habitat improving water quality, access to lost habitat } \\
\text { types and processes, potential removal of invasive plant } \\
\text { species. }\end{array}$ \\
\hline & Dike breaching & $\begin{array}{l}\text { Provides similar benefits as tide gate removal, this } \\
\text { application requires significant earth moving activities to } \\
\text { allow tidal energy to influence historic slough signatures } \\
\text { and can involve tidal channel excavation (see below). }\end{array}$ \\
\hline & $\begin{array}{l}\text { Culvert upgrades/culvert } \\
\text { installation }\end{array}$ & $\begin{array}{l}\text { Provides similar benefits to above restoration activities } \\
\text { through the improvement of water quality, access to lost } \\
\text { habitat types and processes, and potential removal of } \\
\text { invasive species. }\end{array}$ \\
\hline & Elevation adjustment & $\begin{array}{l}\text { Restores elevation of site to level that will support } \\
\text { appropriate wetland vegetation. }\end{array}$ \\
\hline \multirow[t]{2}{*}{ Protection } & Land acquisition & $\begin{array}{l}\text { Preserves existing intact ecological features, functions, } \\
\text { and processes at site scale and/or enables the application } \\
\text { of additional applications without human land use } \\
\text { constraints. }\end{array}$ \\
\hline & Land use regulations & $\begin{array}{l}\text { Limits or prohibits potentially harmful land use activities } \\
\text { on or adjacent to the land surrounding the site, thereby } \\
\text { protecting habitat-forming processes and features. }\end{array}$ \\
\hline
\end{tabular}




\subsubsection{Conservation}

A conservation strategy may be the broadest of all the strategies listed in this document. Conservation measures can be manifested in an extensive range of applications from large-scale sustainable ecosystem initiatives to riparian setbacks to land use ordinances to a streamside, reachspecific conservation easements (Table 3.1). The intention of all conservation strategies is to protect critical ecological features through the application of alternative land management practices. These practices are geared toward the goal of increasing the potential for natural processes to work for the benefit of multiple species. Conservation strategies can be a critical component of a larger restoration plan complementing other restoration strategies that can help increase the site's potential for self maintenance (Section 3.2) by limiting the delivery of harmful inputs from conventional management practices. Conservation strategies frequently include direct payments or other financial incentive to the landowner intended to offset any economic loss resulting from managing the land for conservation. Examples of conservation incentives include financial support for the implementation of riparian setbacks, improved agricultural practices, such as the improvement of manure management practices, the addition of riparian buffer strips, integrated pest management, and off-stream livestock watering techniques. The conservation strategy is especially applicable in the Mixing Zone and Cathlamet Bay Subareas (Table 2.18).

\subsubsection{Creation}

Habitat creation involves constructing or placing habitat features that did not exist previously, but which attempt to mimic conditions of an intact, functioning ecosystem. Tidal channel excavation is an example of a habitat creation strategy intended to replicate the natural structure and function of an intact channel in close proximity to the project site (Table 3.1). Another example of habitat creation activities that have taken place in other coastal systems is the placement of dredge material intended to create marsh or other habitat. Both of these strategies are being considered in the region as possible actions. As mentioned in Section 2.1.3, habitat creation represents the most experimental approach and, therefore, is likely to have a lower degree of success. Because of this uncertainty about the potential ecological gain from a habitat creation strategy, it needs to be complemented by a strong effectiveness monitoring component. Subareas that are being scoped for the application of this strategy include Cathlamet Bay and some of the mid-channel islands in the Tidal Freshwater Subsystem. In addition, creation may be applicable to Youngs, Baker, and Grays bays and the Upper Estuary Subareas (Table 2.18).

\subsubsection{Enhancement}

Habitat enhancement entails the improvement of a targeted ecological attribute and/or process. Several groups are implementing enhancement projects to improve different elements of the ecosystem including: riparian plantings and fencing; tide gate or culvert replacement; invasive species removal; and streambank stabilization (Table 3.1). The Subareas where enhancement activities have been completed or are currently being planned include Baker Bay, Youngs Bay, Cathlamet Bay, and Tidal Freshwater (Clatskanie Flats). In addition, enhancement may be useful in the Entrance, Cathlamet Bay, Grays Bay, and Upper Estuary Subareas (Table 2.18).

\subsubsection{Restoration}

Like habitat enhancement projects, restoration projects can take place in a variety of ways (Table 3.1). According to the working definition (Section 2.1.3), restoration means the return to a 
previously existing ecological condition. This can involve more intense modification and manipulation of site conditions than enhancement. As a result, restoration projects typically require more careful planning, design, and maintenance than enhancement projects. In Section 2, emphasis was placed on the importance of historic wetland habitat types in the life cycle of native salmonid stocks. Miles of habitat can be gained by reconnecting tidal channels that have been cut off by tidewaters, dike construction, and placement of fill material for land-use activities. Reconnecting these areas through the removal of tide gate structures, dike breaching, and or culvert installation into a roadbed, however, can increase the risk of flooding landowners that were previously protected by these structures. Therefore reconnection of tidal channels may require a combination of strategies, such as acquisition and enhancement. At sites where reconnection is not possible, self-regulating tide gates should be considered. CRE subareas where the restoration strategy has the best likelihood of success include the Mixing Zone, Youngs Bay, Grays Bay, and Cathlamet Bay (Table 2.18). Depending on the circumstances, restoration may be a feasible strategy in other subareas as well.

\subsubsection{Protection}

Habitat protection projects can involve a variety of approaches (Table 3.1), but the most common is land acquisition. Another option is to invoke land use regulations in the form of zoning designation and/or protection ordinances, such as defined riparian setbacks and designation of critical areas. Several organizations in the study area are applying these techniques to acquire ownership or development rights to intact patches of habitat or critical areas in need of further restoration treatments. Land use regulations that can be applied are included in comprehensive plans, shoreline management master programs, floodplain management plans, and coastal zone management plans. Table 2.18 indicates that the protection strategy is recommended for the mixing zone but it should be noted that protection strategies are possible and, in fact, are either underway or under consideration in Baker Bay, Youngs Bay, Grays Bay, and the Tidal Freshwater Subsystem (Scappoose Bay).

\subsubsection{Brief Summary of Selected CRE Restoration Projects}

In the CRE, there are several examples of completed, ongoing, or planned restoration projects (Table 3.2). For example, the Blind Slough project in the town of Brownsmead will attempt reconnecting seven miles of slough habitat by installing culverts in road beds and breeching dikes. In Baker Bay, a multiple party effort in the Chinook River basin has conducted extensive hydrologic and hydrodynamic modeling to predict several flooding scenarios resulting from enhancing its connection to Baker Bay. This project also involves the application of protection strategies in the form of land acquisitions and easements, thereby allowing a more pronounced tidal influence into the Chinook system. At Crims Island in the Tidal Freshwater Subsystem, a channel excavation project was funded recently. Additionally, CRE habitat restoration funding is earmarked through several programs, including the U.S. Environmental Protection Agency (EPA) Watershed Initiative Program (grant to the Estuary Partnership), the Northwest Power Planning Council's Fish and Wildlife Program, and the COE's Section 536 Program. Ideally these initiatives will consider the success of the existing, established working relationships between the interested parties. 
Table 3.2. Selected Completed and Ongoing Restoration Projects in the CRE.

\begin{tabular}{|c|c|c|c|c|}
\hline Project & Subarea & Strategies & Type of Activities & Project Phase \\
\hline Blind Slough & Cathlamet Bay & $\begin{array}{l}\text { enhancement, } \\
\text { restoration, } \\
\text { conservation }\end{array}$ & $\begin{array}{l}\text { culvert installation, dike } \\
\text { breach, manure } \\
\text { management }\end{array}$ & $\begin{array}{l}\text { planning, effectiveness } \\
\text { monitoring (scheduled for } \\
\text { construction-2004) }\end{array}$ \\
\hline Chinook Estuary & Baker Bay & $\begin{array}{l}\text { restoration, } \\
\text { protection }\end{array}$ & $\begin{array}{l}\text { tide gate removal, land } \\
\text { acquisition }\end{array}$ & $\begin{array}{l}\text { planning, effectiveness } \\
\text { monitoring }\end{array}$ \\
\hline Crims Island & Tidal Freshwater & $\begin{array}{l}\text { restoration, } \\
\text { protection }\end{array}$ & $\begin{array}{l}\text { channel excavation, land } \\
\text { acquisition }\end{array}$ & planning \\
\hline Grays River & Grays Bay & $\begin{array}{l}\text { restoration, } \\
\text { protection }\end{array}$ & $\begin{array}{l}\text { dike breeching, land } \\
\text { acquisition }\end{array}$ & completed in 2002 \\
\hline $\begin{array}{l}\text { Port of Astoria } \\
\text { Mitigation Bank }\end{array}$ & Youngs Bay & $\begin{array}{l}\text { restoration, } \\
\text { enhancement }\end{array}$ & $\begin{array}{l}\text { dike breach, invasive } \\
\text { species removal }\end{array}$ & $\begin{array}{l}\text { completed in } 1989 \text {, } \\
\text { effectiveness monitoring, } \\
\text { adaptive management }\end{array}$ \\
\hline $\begin{array}{l}\text { Skipanon River- } \\
\text { 8th St. Dam }\end{array}$ & Youngs Bay & enhancement & tide gate replacement & $\begin{array}{l}\text { completed in } 2002 \text {, } \\
\text { effectiveness monitoring, } \\
\text { adaptive management }\end{array}$ \\
\hline Trestle Bay & Entrance & restoration & trestle removal & completed in 1995 \\
\hline $\begin{array}{l}\text { Youngs Bay, Tide } \\
\text { gate }\end{array}$ & Youngs Bay & $\begin{array}{l}\text { enhancement, } \\
\text { conservation }\end{array}$ & $\begin{array}{l}\text { tide gate replacement, } \\
\text { riparian plantings }\end{array}$ & completed in 2002 \\
\hline
\end{tabular}

\subsection{Project Selection}

\subsubsection{Review of Prioritization Approaches}

There is no universally accepted method to prioritize projects (sites) for restoration. At a national level, the COE (2000) has the most developed planning process for projects under their civil works mission (i.e., navigation, flood control), and they are adapting this process to ecosystem restoration projects (Thom et al. in press). Once a site is selected, the COE process evaluates alternative plans relative to environmental planning objectives and cost. Through what is termed "incremental costeffectiveness analysis, they arrive at a point where there is a rapidly diminishing return on investment in the project. The process highlights the action that provides the most return per unit of investment. The COE utilizes environmental indices as metrics to evaluate environmental outcomes from alternative restoration plans (e.g., habitat suitability indices; hydrogeomorphic indices; Shafer and Yozzo 1998; Thom et al. in press).

In the Pacific Northwest, several approaches have been applied to prioritizing restoration projects. The approaches have common elements: a goal statement; a site assessment to ascertain changes in conditions from historical; a set of selection criteria; and a qualitative or semi-quantitative scoring protocol. The overall driver for these programs is to identify optimal actions that will result in improved conditions relative to the goal. Improvement in the landscape/watershed (e.g., limiting factor analysis) is a site selection factor. However, opportunity for restoration (i.e., the site is made available, such as the Blind Slough Project) at least partially drives the process of site prioritization.

In highly urbanized and developed estuary areas, site selection and prioritization is strongly driven by the cost for the site and its restoration relative to the chance for restoration to be successful (Shreffler 
and Thom 1993). The chief drawback with all prioritization approaches has been the need to rely heavily on subjective information (i.e., expert opinion) in lieu of data. Objective and unbiased methods are desired, but critical data on key ecological relationships to accomplish this are usually lacking. For example, it would be ideal to develop a metric that indicates the increase in fitness of juvenile salmon relative to various manipulations of the CRE ecosystem. Because this is not possible with our present understanding, people utilize surrogates such as area of selected habitats, juvenile salmon prey densities produced by habitats, area covered by exotic plants, and area of intact riparian zone. There are modeling efforts underway (e.g., Greene et al. 2003; Kareiva et al. 2000) which demonstrate that small increases in survival in estuarine and nearshore areas in the Pacific Northwest would have the greatest relative effect on adult salmon returns relative all other habitats used by salmon.

In developing ecological assessment criteria for restoring anadromous salmon habitat, Simenstad and Cordell (2000) advocated the use of measures directly relatable to the ecological and physiological responses of juvenile salmonids to restored habitats. They proposed the use of three categories capacity, opportunity, and realized functions (Table 3.3). Capacity metrics include habitat attributes that promote juvenile salmon production through promotion of foraging, growth, and growth efficiency, and/or decreased mortality. The capacity category is an extension of the ecological concept of carrying capacity. Examples of capacity metrics include the productivity and density of prey, physical and chemical conditions that promote high assimilation efficiencies, and structural conditions that provide protection from predation. Opportunity metrics appraise the ability of salmon to access and benefit from the habitat's capacity (Simenstad and Cordell 2000). Opportunity incorporates the principles of landscape ecology (Forman and Godron 1986). Examples of metrics include tidal elevation of feeding habitats, and the extent of morphometric features such as habitat edge length, and refuge from predation (such as low tide deep-water refuges). Finally, realized function metrics include any direct measures of physiological or behavioral responses that can be attributable to fish occupation of the habitat and that promote fitness and survival (Simenstad and Cordell 2000). Survival is the ultimate metric, but related metrics include habitat-specific residence time, foraging success and growth.

Table 3.3. Category of metrics used to assess effects of shoreline armoring on salmonid growth and survival (summarized from Simenstad and Cordell 2000).

\begin{tabular}{|c|c|c|}
\hline Category & Alteration & Potential Impact to Salmon \\
\hline \multirow[t]{5}{*}{ Capacity } & Altered habitat type & Change in prey species \\
\hline & Altered habitat forming processes & Change in prey production \\
\hline & Altered habitat production & Change in prey abundance \\
\hline & & Change in prey distribution \\
\hline & & Change in predator abundance \\
\hline \multirow[t]{5}{*}{ Opportunity } & Altered access & Change in ability to find prey \\
\hline & Altered migration route & Change in rate of migration \\
\hline & Altered habitat size & Change in predation rate \\
\hline & Altered habitat location & Change in predation rate \\
\hline & Altered refuge from predators & Change in ability to avoid predators \\
\hline \multirow[t]{2}{*}{ Realized Function } & Altered residence time & Change in growth rate and survival \\
\hline & Altered foraging success & Change in foraging success \\
\hline
\end{tabular}




\subsubsection{Multi-Criteria Methods}

Multi-criteria methods use data on the physical and chemical requirements (i.e., the controlling factors as used in this report) of a selected habitat (e.g., tidal marshes) along with data on past restoration experience for that habitat to parameterize a model or index that evaluates the restoration potential for sites in a region (e.g., Shafer and Yozzo 1998; Store and Kangas 2001; Short 2003). Recent work with multi-criteria methods link results directly to a GIS where the results of the analysis can be displayed on maps of the region (Store and Kangas 2001). The advantage of a Habitat Suitability Model (HSM) is that quantitative data on habitat requirements are used along with information on existing conditions at sites. If quantitative data on habitat requirements are available and used, this type of analysis is generally more objective than other methods relying on qualitative data. Expert opinion, which is susceptible to bias and lack of repeatability, can also be incorporated when quantitative data are not available.

For analysis of sites, the method not only requires information on what a habitat type needs, but also on the historic and present conditions of sites in the region. For example, a site with appropriate conditions prior to development may not presently be suitable for a particular habitat, because the processes that caused degradation of the habitat might not have been corrected. Therefore, careful examination of the potential sites needs to incorporate past and present conditions, as well as the degree of change necessary in order to re-establish the habitat. This method deals only with habitats where there is a large amount of information on their requirements as well as their restoration potential. A separate model would be required for each habitat within a system.

The Index of Biological Integrity (IBI) is a multi-metric index of habitat quality and condition that combines several environmental or biotic variables to evaluate aquatic resources and to assess the effects of anthropogenic degradation (Karr 1993; Hughes et al. 2002). A biotic index is calculated based on a set of measurable biotic variables that are known to be indicative of habitat quality. For example, Hughes et al. (2002) used the following set of variables to evaluate estuarine quality on the East Coast: fish abundance or biomass; total fish species per trawl; species dominance; number of resident species; number of estuarine nursery species; number of in-estuary spawning species entering the estuary as adults to spawn; proportion of benthic-associated, or demersal, species; and proportion of diseased fish.

The eight IBI variables are compared to critical values indicating low habitat quality and assigned a score. In this example, if the value was above the critical value, the metric was given a score of 5 . If the value was below the critical value it was given a score of 0 . Because the method of sampling was a trawl, the scores are summed for each trawl sample, with the maximum score for a trawl being 40. Often an independent set of data on water quality or other environmental variables is collected, computed as an index similarly to the IBI, and compared to the IBI index scores. If the IBI index is a valid indicator of habitat conditions, the IBI score will correlate with the index based on environmental variables. Through analysis, the environmental factors most responsible for site-to-site variation in the IBI can be identified and these can guide actions at the site that would lead to an improved IBI. In order for the IBI analysis to be the most informative and defensible, critical values for the biotic and abiotic variables need to be known.

\subsubsection{Relevance to the CRE}

In the CRE, a numerical multi-criterion assessment of habitat suitability could be developed for juvenile salmon habitat. Using elevations for habitat presumably used by fish and current velocities, Bottom et al. (2001) provide a fundamental set of metrics that should be considered. Circulation 
modeling is helping define where in the estuary the range of conditions for depth and current velocity occur. New research is trying to refine the metrics for fish to determine whether improvement of conditions actually translates into higher growth and survival. A drawback to relying solely on this approach is that it constrains the restoration process to concentrate only on fish survival as the end product.

A numerical multi-criterion assessment of habitat suitability could be developed for vegetated systems including tidal swamps and tidal marshes. However, for the CRE there is limited quantitative information on physical and chemical requirements for these habitats that would drive assessments of the appropriateness of sites for restoration. Although data are lacking to apply an IBI multi-metric analysis in the CRE, this type of assessment may be appropriate for evaluating the functionality of restoration projects. For example, an index based on a suite of fish species caught in beach seines at restored and reference sites would indicate how well the restored site is functioning. An IBI approach could also be employed as a monitoring tool to compare conditions before and after site restoration to determine the relative effects of restoration actions.

The process recommended here relies on solid ecological principles, coupled with the best available scientific understanding of the ecosystems in the Pacific Northwest and the best information available on the biophyscial conditions of the estuarine habitat conditions in the CRE. Specifically, the process developed here relies on restoration of controlling factors as the key to successful and long-term restoration. Because of this, it will be important for restoration planners and managers to understand the extent to which key controlling factors can be modified, e.g., river discharge patterns. In addition, basic information on controlling factors and their effects on juvenile salmon habitat in CRE reaches may be insufficient to fully evaluate the appropriate strategy and potential for a strategy to work for those reaches. As knowledge and experience are gained, CRE restoration strategies should be revised as appropriate. In some instances, site-specific evaluations would be required to understand what factors need to be restored and to what level.

\subsubsection{General Prioritization Method for CRE Restoration Projects}

The prioritization involves an initial assessment of what strategies would have the highest success potential within each subarea, followed by a site-specific assessment to refine the strategy. This approach uses landscape ecology and conservation biology principles, and national recommendations on the most applicable restoration strategies as the fundamental underpinnings for prioritization (see Section 2.1; NRC 1992; Shreffler and Thom 1993). These principles are well established in the ecological literature and provide highly useful, comprehensive, large-scale guidance.

\subsubsection{CRE Habitat Conservation and Restoration Workshop Guidance for Project Selection}

There are criteria developed specifically for the CRE restoration that should be considered in site selection and prioritization. In June 2001, the COE, Estuary Partnership, and others convened a two-day workshop in Astoria, Oregon to develop science based criteria for identifying and prioritizing habitat protection and restoration projects in the CRE (COE et al. 2001). The workshop participants worked from the premise that there was a need to move ahead with restoration actions in spite of limited knowledge about the ecosystem. The workshop developed six "themes," which provide guidance for selection and prioritization of projects in the system. The six themes are: 
- Habitat connectivity - Emphasis should be placed on connectivity among habitats to provide species areas to disperse and survive and to provide access to areas of high quality habitat especially valuable to juvenile salmon

- Areas of historic habitat loss - Areas where habitat loss has been greatest should be considered for restoration, depending on the nature of the loss and current uses at the site. These areas include forested and emergent wetland types that serve salmonids and birds.

- $\quad$ Linkages to reference sites - Sites should be chosen that include an experimental restoration design tied to effectiveness monitoring to promote a better understanding of the relationships between habitat restoration activities and species response and performance resulting from the restoration activity.

- Passive habitat restoration over creation -Areas where minor alterations would be needed to maximize ecosystem function should be prioritized over areas where massive alterations or creation of new ecosystems would be required. The groups identified conservation of existing natural areas under this theme.

- $\quad$ Monitoring and evaluation - Sites should be selected that lend themselves to an effectiveness monitoring program. Information on limiting factors and properly functioning conditions should be included in the site selection and project design.

- $\quad$ Community support and participation -sites should be selected that can maximize local community support and participation.

\subsubsection{Additional Development of Project Selection Guidelines}

The Astoria workshop providing a useful step in the development of project selection guidelines, but further analysis is required to define the best restoration action for each subarea or site (i.e., a portion of a subarea where a management decision is required). To foster this additional analysis, we reviewed ecological restoration literature. There are eight generally accepted guidelines to guide the development of restoration actions. The first four are based on landscape ecology and conservation biology principles and indicate the existing environmental quality of the reach. The latter four are based on restoration ecology and reflect the potential environmental quality of the reach following implementation of a habitat action strategy. The focus of the latter criteria is on the potential for controlling factors to be reestablished or enhanced at the site (reach) that would eventually result in a structurally and functionally enhanced and sustainable ecosystem. These eight guidelines incorporate the principles developed in the 2001 Astoria workshop for the CRE. Together these criteria and workshop principles can provide guidance in the development of a site design plan and project selection guidelines. The eight guidelines are organized into two categories, Existing Environmental Quality and Potential Environmental Quality, as follows: 


\section{Existing Environmental Quality}

- $\underline{\text { Size }}$ - Size refers here to reach length and the size of the potential habitat within a reach. In general, larger size enhances habitat stability, increases the number of species that can potentially use the site, makes it easier to find by migratory species, and increases within-habitat complexity. This is related to the SARE criterion of opportunity and capacity.

- Complexity - This criterion refers to the numbers of different types of habitats within a reach. As the number of habitat types increases, so does the number of species that can occupy the area, and the number of functions supported by the area. Higher complexity potentially results in greater biodiversity. This is related to the SARE criterion of opportunity and capacity.

- $\quad$ Accessibility - Accessibility refers to unencumbered access by CRE habitat-dependent aquatic and terrestrial species. Projects that would allow or enhance access of these species to important habitats would potentially enhance the feeding, rearing, and refuge functions of the site. This is related to the SARE criterion of opportunity.

- Connectivity - This criterion refers to the degree of natural connection and pathways between adjacent habitats or migratory corridors. Connectivity means that an animal can move between adjacent habitats to derive the benefits of each habitat. It also refers to the flow of material such as organic matter between areas of production (e.g., a salt marsh) and areas of deposition (e.g., tidal channels and creek bottom where the materials are utilized by the ecosystem). This guideline represents the "habitat connectivity" theme developed by the Astoria workshop (COE et al. 2001).

\section{Potential Environmental Quality}

- $\quad$ Potential to conform to natural habitat structure, processes, and functions - This criterion expresses the relative probability that a site can return some or all of the natural habitat structure, function and processes found on the site historically. As mentioned above, the level of site and landscape disturbance is important. This guideline represents the "areas of historic habitat type loss," "passive habitat restoration over habitat creation," and "linkages to reference sites" themes developed by the Astoria workshop (COE et al. 2001).

- Potential for self-maintenance - Self-maintenance addresses the ability of a site to persist and evolve toward a natural (historical) habitat condition without significant human intervention. As a pre-requisite for this to occur, conditions for controlling factors in the reach and in the management unit must be appropriately developed and maintained. Self-maintenance means that the habitat can persist and develop under natural climatic variation, and that the system has a natural degree of resilience to natural perturbations. This criterion also takes into account the need to know the probable historical conditions, and the factors that produced the present conditions. This guideline represents the "areas of historic habitat type loss" theme.

- $\quad$ Potential benefit to nearshore-dependent threatened and endangered species - This criterion is specifically directed at those species whose populations are at precariously low numbers and who might benefit from improved nearshore habitat conditions. At present, wild Chinook salmon would be one of the major species to benefit from improved nearshore conditions. This guideline coincides with themes from the Astoria workshop and SARE. 
- Potential to substantially improve ecosystem functions - This criterion acknowledges that some actions can result in greater enhancement of ecosystem functions than others. These projects may not be the largest or most complex projects. For example, the location may be more important than the size of a project. A medium-sized project in a location where an endangered species can directly benefit because of the proximity to its normal migratory pathway would be more important than a project far outside of the pathway. This guideline links to all themes from the Astoria workshop and SARE.

A ninth guideline is based on a practical concern for habitat restoration. Land must be available to restore. If land is not available, then the project cannot proceed. Therefore, land availability is an important project selection criterion.

The nine guidelines, summarized in Table 3.4, are for the most part qualitative. They can be applied directly to evaluate the benefit of management action alternatives for a particular site. A simple scorecard could be used to apply these guidelines. For example, with the possible exception of size (which can be stated as areal extent of habitat types within a reach or site), each of the remaining seven guidelines can simply be assessed as being absent or present or something in between, i.e., on a qualitative scale of 0 (poor), 1 (acceptable), or 2 (good), with a qualifying comment as to degree.

Table 3.4. Project Selection Guidelines and Scorecard. Based on Astoria Workshop (COE et al. 2001), SARE (Bottom et al. 2001), and Williams et al. (2003).

\begin{tabular}{|c|c|c|c|}
\hline No. & Description & Score & Comment \\
\hline 1 & Adequate size & & \\
\hline 2 & Level of complexity & & \\
\hline 3 & Accessibility & & \\
\hline 4 & Connectivity & & \\
\hline 5 & Potential to conform to natural habitat structure, processes, and functions & & \\
\hline 6 & Potential for self-maintenance & & \\
\hline 7 & Potential benefit to threatened and endangered species & & \\
\hline 8 & Potential for improvement in ecosystem function & & \\
\hline 9 & Land availability & & \\
\hline Total & Sum of scores & & \\
\hline
\end{tabular}

Guidelines 5 and 6 can be assessed relative to the level or degree of disturbance on the zone scale. An appropriate question would be, "Are the natural habitat-forming processes healthy enough to allow for the development and maintenance of natural habitat structure and functions, and will the site be maintained through time?" Multi-criteria methods available, but still untested in the CRE, could also be used for specific habitat recommendations addressing Guidelines 5 and 6.

To address Guideline 8 relative to salmon, the criteria of Simenstad and Cordell (2000) and SARE (Bottom et al. 2001) can be applied (Table 3.3). Will the management action potentially provide enhanced capacity, opportunity and realized function in support of juvenile salmon? Guideline 8 can be evaluated by examining the present CRE subsystem functional level (Figure 2.1) and whether this functional level can be enhanced to another level (state). In some cases the functional state may be high 
for sites within subareas that have undergone moderate disturbance, and improvement to functional level may not be possible, even with improved conditions in the reach-controlling factors.

In summary, after a broad project concept is outlined, it is evaluated against the priority restoration strategies for that particular subarea (Table 3.1) and the project selection guidelines (Table 3.4). This approach will reduce the degree of uncertainty for potential gain of habitat function upon completion of the project, i.e., the potential for successful implementation. It is recommended that these guidelines (Table 3.4) provide the basis for prioritizing projects in order to embody fundamental ecological considerations. Project selection is a key element of the Planning Phase in the project process.

\subsection{Project Phases}

Once a possible project has passed the basic criteria, it will evolve through a series of phases that includes a combination of the strategies and guidelines detailed in this document. Every project will inherently possess unique idiosyncrasies and challenges. The project phases described herein embody the scientific principles of this approach and are meant as a guide for project sponsors. The fundamental phases of a restoration project are planning; implementation; effectiveness monitoring; and adaptive management. A special worksheet that could serve as a template for project implementation is included below in Table 3.5. A narrative follows that provides details for restoration project phases. The overall process follows the principles of adaptive management and is explained by Diefenderfer et al. (2003).

\subsubsection{Planning}

Restoration projects originate in the planning phase. It is here that the restoration strategies recommended in Section 2.5 are applied for specific CRE types of projects presented in Section 3.1 and prioritized in Section 3.2. In general, the planning phase includes the following steps: site selection; goals and objectives; coordination and outreach, engineering and design; and funding. This section describes a stepwise approach to plan a restoration project.

Site Selection and Assessment: The first step of the planning phase is site selection and assessment. Project sponsors through their relationship with the local community or other initiatives (Appendix A) will have a list of prospective sites to consider. Maps that include historic and current habitat type distribution may also direct attention toward sites in a particular subarea. Applying historic habitat information presented in Section 2.4 might help the restoration planner direct an initial list of recommended strategies for the subarea based on landscape level considerations. At the site-specific scale, the Action Agencies could begin to apply the project selection guidelines described above (Table 3.4; Section 3.2). These guidelines will help determine a site's ecological value and serve as an initial filter to reduce the uncertainty about the project's potential to improve ecological functionality. This helps assure that fundamental ecological components are in place before the opportunity is investigated further. Another relevant piece of information to consider during the planning phase is watershed assessment data. These data provide current information on water quality, channel habitat types, and usage by aquatic species. Furthermore, deficiencies in the knowledge base in can form the basis for recommending the collection of additional baseline information. 
Table 3.5. An Adaptive Management Framework for Restoration Project Phases, Activities, Suggested Tools, and Pertinent Sections in the Plan. While the process depicted in this table is not linear, it is intended to show the progression loop of activities.

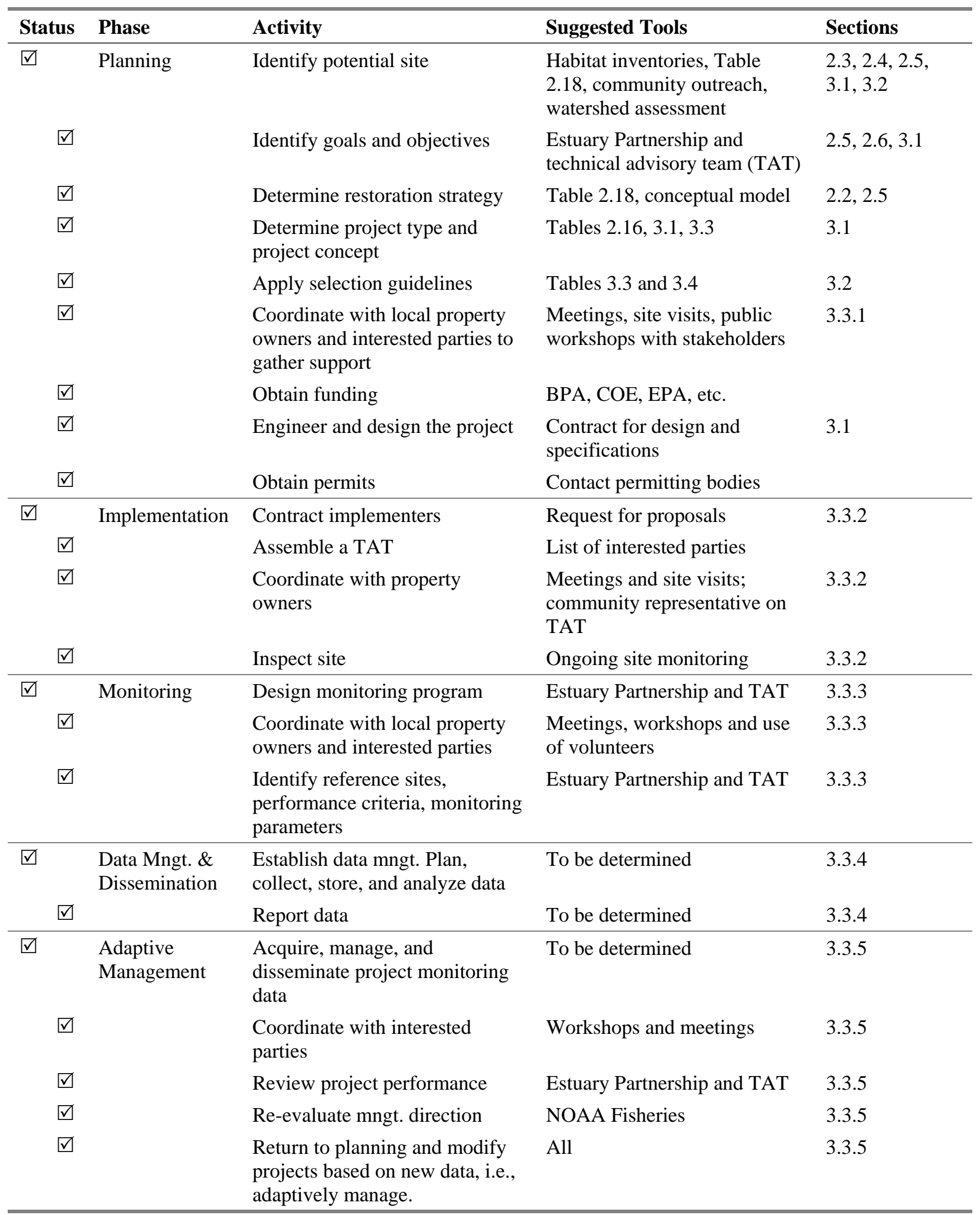


In addition, further evaluation of a potential restoration site is frequently needed to define specific actions required to restore the site. For example, a dike breach around a former tidal wetland may be the recommended action for a site, but based on application of the project selection guidelines the elevation of the land contained within the dike may be far too low to allow the immediate colonization of wetland plants. Typically, the land surface subsides following diking. In some cases subsidence can be substantial (e.g., 1-3 m), which may preclude any vegetation colonization until natural sedimentation processes raise the elevation into the range where plants can survive. To facilitate vegetation colonization, placement of sediments may be necessary. Site assessments provide the specific information needed to evaluate the factors responsible for the loss of habitats at the site, and what is necessary to restore the habitats. At priority wetland-channel system restoration sites, determine surface and subsurface properties, including land topography and channel bathymetry, vegetation assemblages, stratigraphy, soil and sediment characteristics, and hydrologic features. It is strongly recommended that site assessment be carried out for every project. The aggregate of available information during the site selection and assessment process provides a preliminary scientific basis for the next step in the Planning Phase: Goals and Objectives.

Goals and Objectives: Building on information compiled during the Site Selection and Assessment, the restoration planner should begin developing an overall project goal and measurable objectives to achieve the goals. Goals should embody components of the CRE conceptual model (Section 2.2) and controlling factors and associated alterations (Table 2.16). This process will assist the project direction based on landscape elements affecting specific habitats to the CRE. It also will show how the selected habitats, if restored, could influence other ecosystem components. For example, if a restoration project involves reconnecting a cut-off slough to tidal waters, an obvious restoration goal relates directly to the controlling factor listed in Table 2.16 as hydrodynamics. According to the conceptual model, a modification in hydrodynamics results in changes of multiple components, such as suspended sediment and bed load. This then can change the elevation and turbidity, which in turn are factors in habitat type formation and distribution, etc. Therefore, once a clear goal has been established, objectives to achieve it should be developed based on existing data and measurable ecological parameters. Each objective should entail specific activities designed to achieve the objective. The process of developing goals and objectives helps form a basic project design by adding details to the original project concept. The individual project goals should be linked to the goals for the CRE habitat restoration program.

Coordination and Outreach: Coordination and outreach with the local community of stakeholders is an important parallel process that should occur during the planning phase. Project sponsors need to develop strong connections and support in the local community to ensure long-term project success. Many of the existing groups conducting restoration in the study area perform extensive outreach to the local community to assess project feasibility in the context of community needs. Project sponsors should be familiar with the local conditions of the site and have developed relationships with community leaders and landowners. This is a great asset during this phase of project implementation, especially in the development of such things as landowner agreements that provide written testimony of stakeholder's expectations upon project completion. Because of their perspective and experience, local stakeholders often have innovative ideas for solutions to problems that arise during project development. Project sponsors are in the unique position of making iterations from previously defined goals, objectives, and strategies as they are nested in the context of stakeholder concerns and needs. Project activities will likely need to be refined again and again as the project is engineered and gets closer to the construction phase. This helps ensure that the project will not be compromised. 
Engineering, Design, and Costing: This step in the planning phase varies greatly depending on specific site conditions, the composition of strategies, available data, and funding constraints. This marks a critical next step in the process as planners move from a conceptual idea for a site to the mechanics of what is feasible based on resources for engineering, designs, and specifications. In the CRE, there may be insufficient data to adequately predict how the application of the strategies in the previous step will alter existing conditions. To narrow the risk associated with implementing large-scale restoration strategies with insufficient data, actions such as tide gate removal and/or dike breaching in the Estuarine Subsystem may require hydrologic and hydrodynamic modeling to better anticipate the range of tidal-fluvial responses for a selected restoration alternative. This may involve the collection of additional data to adequately run the model based on existing conditions. The results of the modeling can guide the design and help determine the types of materials to be used for construction and their estimated costs. Moreover, modeling can also be a vital step for a project sponsor when assessing the possible success of the project in light of project goals. For example, while removing a tide gate may be the most desired alternative for providing the highest ecological benefit, it may come with additional costs in the form of land acquisition and/or construction of new dikes to protect landowners. This cost must be weighed with other restoration alternatives such as a tide gate replacement, which, while providing less ecological gain, may be more feasible in meeting funding constraints and the concerns of the community.

Funding: The work accomplished during the planning phase is critical to the ultimate approval of the project and the securing of funding. Projects proposed for funding should be consistent with the scientific principles and implementation process articulated in this document. A variety of sources may be able to provide funding including: the BPA's implementation of the Northwest Power and Conservation Council's Fish and Wildlife Program; the COE under various authorities (see Appendix A); other federal agencies including NOAA Fisheries, EPA, and USFWS; state agencies including the fish and wildlife agencies, the Washington Salmon Recovery Funding Board, the Oregon Watershed Enhancement Board, and non-governmental granting organizations such as the Estuary Partnership. One of the primary goals of the Estuary Partnership is to work with partners to identify projects and then secure the funding to implement them for coordinated and strategic restoration in the CRE.

\subsubsection{Implementation}

This is the phase when on-the-ground work occurs. Once construction begins, project sponsors should carefully document the process to facilitate transfer of information to aid development of future projects. Communication and coordination mechanisms installed during the planning phase between the project sponsors, the local community, contract administrators, and contractors are most critical during project implementation. A technical advisory team with representatives from all four groups might be formed to track progress and guide any necessary modifications. This group can also serve to leverage additional resources for project contingencies and provide a forum for conflicting interests and concerns that may arise during implementation. Project sponsors should look to technical resources for help such as the Estuary Partnership's Science Work Group, NOAA Fisheries, USFWS, and state fish and wildlife experts.

\subsubsection{Effectiveness Monitoring}

Habitat restoration in the CRE ecosystem and other systems nationwide as well, is a relatively new field. The anticipated ecological benefits identified during the planning phase are anything but certain. Put simply, the term "successful restoration" is not uniformly understood or agreed upon. Establishing 
performance criteria (defined below) to track ecosystem response to restoration treatments in the past has been viewed largely as an afterthought. This is reflected in the relatively low percentage of total project funds dedicated to monitoring during project implementation. Only recently has monitoring been included as a component of CRE restoration work, e.g., the Blind Slough and Chinook projects. For more basic information on effectiveness monitoring, see Thom and Wellman (1996) and Busch and Trexler (2003).

Monitoring represents the critical step towards understanding the CRE through testing the previously held assumptions about the ecosystem. It is also critical for adaptive management (see Section 3.3.5). For adaptive management to work in the CRE, effectiveness monitoring needs to be linked to data management and dissemination mechanism in order to communicate restoration project "lessons learned" in a meaningful way to benefit o groups conducting restoration planning. Effectiveness monitoring methods should be based on the scientific literature and large-scale initiatives such as the LCREP Monitoring Strategy (LCREP 1999), the monitoring plan currently being developed by the Estuary/Ocean Subgroup for Research, Monitoring, and Evaluation, and the major regional programs described in Section 1.5. While monitoring plan development will vary according to project goals and objectives, there are fundamental considerations including performance criteria, reference sites, and monitoring methods.

Performance Criteria: Preliminary development of an effectiveness monitoring plan begins during the development of project goals and objectives. If the goals are clear they can be easily reworded into a set of testable hypotheses. These hypotheses could then form the basis for what are called performance criteria. Performance criteria are defined as "standards by which to evaluate measurable or otherwise observable aspects of the restored system and thereby indicate the progress of the system toward meeting the project goals" (Thom 1993). The performance criteria can involve a variety of biological and physical parameters. Deciding which parameters are most relevant is directly related to the project's objectives. The project sponsor must ask the question: What parameters will we be able to measure to effectively track of the project's progress toward achieving its stated goal(s)? Monitoring parameters and sampling design will depend on project goals. Project sponsors will need to prioritize the necessary components of the monitoring plan and weigh that against their organization's existing capacity and project funds. It is critical that sufficient monitoring be completed to measure a project's effectiveness and success. Project performance should also be tracked collectively relative to the CRE habitat restoration program goals.

Another constraint on developing an effectiveness monitoring plan is defining an adequate time frame. Positive ecological benefits from a restoration project in the form of quantifiable results are often difficult to demonstrate in the short term. Developing useful performance criteria given natural variability presents another challenge for project sponsors. Finally, when developing performance criteria, it is assumed that once they have been met, the ecosystem is able to maintain itself in perpetuity but this may not be the case, which means that some basic ongoing monitoring should be built into the project.

Reference Site Selection: A common definition of a wetland reference site is given by Brinson (1993) as a "Wetland or one of a group of wetlands within a relatively homogeneous biogeographical region that represents typical, representative, or common examples of a particular hydrogeomorphic wetland type, or examples of altered states." During the site selection phase, general habitat criteria (Section 3.2) were applied to restoration opportunities that were located adjacent to an appropriate 
reference site. A reference site nearby provides a project outcome target as well as the basis for a good monitoring plan. Incorporating a reference site into an effectiveness monitoring plan helps predict the natural range of ecological conditions expected from a treated site. Projects sponsors are strongly encouraged to apply this standard as part of their monitoring effort. Finding reference site candidates for an adequate paired comparison is challenging given the scarcity of intact functioning habitat patches in the CRE. Failing that, a degraded reference site can also perform a role to demonstrate the progress of the treated site away from the degraded condition.

Selection of Monitoring Parameters and Methods: Formulation of monitoring parameters will depend in part on the project's goals and objectives. According to NRC (1992) recommendations, monitoring parameters should be selected based on the general conceptual model presented in Section 2.2. There are many parameters for monitoring ecological performance related to restoration. Recall that these parameters can be categorized under three different classes: controlling; structural; and functional. A strong monitoring plan should attempt to select parameters from each class. An example of these parameters would be tide level range (controlling), length of marsh channel interface and tidal marsh area (structural), and juvenile salmon prey density (functional). See Thom and Wellman (1996) and Busch and Trexler (2003) for more details on complexity, duration, quality assurance, data management, field sampling protocols, laboratory sample analysis protocols, data analysis and interpretation, report preparation, presentation of results, and complimentary data sets.

\subsubsection{Data Management and Dissemination}

To manage and disseminate existing and new restoration project data (project inventory, project status, effectiveness monitoring results, etc.), an information center is needed to house, store, analyze, and disseminate data on the CRE. Such an effort would build a data management system to provide on-line access to all data on the CRE in a variety of formats including raw data, processed data in report form, customized data as needed, and on-line assistance to pinpoint data needs and expedite access for users. A CRE information center would be the culmination of the information management strategy envisioned in the "Lower Columbia River Estuary Plan" (LCREP 1999). It would encompass the needs of the multitude of organizations that are working in collaboration to address salmonid recovery and restoration the CRE. As part of this effort, the information center would develop data sharing agreements between the multiple organizations that collect data on the lower river so that new information and data would automatically be channeled to the information center and be available to interested parties. The collaborating organizations track the condition of the river over time and would work to develop strategies designed to keep the scientific community, the public, and resource managers apprised of new information developments. Tools such as traveling interactive displays, information access kiosks in strategic public facilities, public workshops, a regularly published "state-of-the-river" index, newsletters, school presentations, and efforts specifically targeted at minority groups would all be employed to reach the largest audience possible. The intent would be to support habitat restoration and salmon recovery efforts in the CRE by providing ready access to such things as habitat maps; interpretation of complex hyperspectral data; physical, chemical, biological, hydrographic, and bathymetric data in raw and processed forms; and project specific information. Regarding data dissemination, the Estuary Partnership's Board of Directors at its July 2003 meeting adopted the task over the next five years to establish a central location for data links. In summary, although a CRE information center is not yet funded, it would be an important asset to the restoration process. 


\subsubsection{Adaptive Management}

An ultimate goal of restoration ecologists is to have a science capable of predicting the structure and function of natural communities and ecosystems, especially those whose component species have had a chance to adapt to whatever perturbation society proposes to impose (Rosenzwieg 1987). The very act of restoration should provide new insight into the functioning of ecosystems. In turn, greater understanding of how ecosystems function will enhance our ability to predictably restore them successfully. This approach of accumulated understanding is termed adaptive management. Adaptive management must be effectively incorporated into restoration projects to help the projects succeed and to improve predictive capabilities (Thom 1997; Thom 2000). Particularly in the CRE, where our knowledge of outcomes is slim, it is critical that we learn as we go, and this can only be accomplished by treating each project as an experiment. As the Independent Scientific Review Panel wrote in comments on a draft, "It is important that adaptive management be understood to be a structured experimental process that is adjusted as information is collected over time, and alternative hypotheses are tested. In a complex, open, and highly dynamic environment such as the estuary, we expect that adaptive management practices could be invaluable in testing various restoration methods and testing how to best assist the recovery of these species."

Restoration technology is continuously developing, and each project can provide much needed information that increases the success of future projects. In addition, each project provides an opportunity to evaluate predictions of the effects of each action, and the rate and trajectory of development of habitat structure and function. Because of the paucity of this type of information, the NRC (1992) has recommended that restoration projects be conducted as experiments. This means that the project is set up with a set of questions, some level of design to control for variables that might confuse results, and an effective assessment program. Manipulations to evaluate questions should be incorporated into projects if they would yield critical insight into important and broader-scale questions.

Whether it is used to change the course of an ongoing project or used on a broader scale to inform the management of future restoration projects in the region, adaptive management represents an important part of the implementation guidelines in providing a needed framework for managing the diversity of projects geared toward the future health of the CRE. Project sponsors who use this approach are encouraged to share their monitoring information and project implementation process by participating in a coordinated infrastructure that embodies the tenets of adaptive management as it applies to restoration. This body can provide an ecosystem perspective and oversight and can develop processes to manage and disseminate the results of projects (see Section 3.3.4) with the goal of improving restoration strategies in the future. The Estuary Partnership's long-term monitoring strategy is an example of the kind of effort needed to tie together the efforts contributing to an overall understanding of ecosystem health for the region. Other major initiatives, such as Action Agency's work to develop and implement a research, monitoring, and evaluation plan in response to the FCRPS BiOp (NMFS 2000), further justifies the need for a mechanism that integrates these efforts with the monitoring efforts of project sponsors. Adaptive management provides the feedback loop from project conception to implementation to monitoring to selection of new projects.

In addition to the variety of restoration-oriented organizations in the area and the variety of proposed projects, various monitoring initiatives are also taking place at various scales, all of which point to the need for enhanced coordination. The specific details on the organization processes are not given here, but there is a need for a body to coordinate restoration activities and to convey restoration and monitoring 
information that contributes to a larger understanding of overall Columbia River estuary ecosystems. A coordinating body would provide the framework within which project sponsors can coordinate with other entities of the restoration community and look at restoration from the landscape level using such things as habitat maps, interpretation of complex hyperspectral data, and updates on current biophysical modeling.

Adaptive management is not complete without evaluation and assessment of the project in a feedback loop to project sponsors. Feedback is where the lessons learned from a particular project are applied to modify future monitoring for that project and to design new projects. Ideally, the cumulative effect of multiple restoration projects will be determined and linked back to project implementation. This would also be under the purview of the coordinating body.

\subsection{Summary}

The Implementation Guidelines are intended to inform a more coordinated approach by presenting a set of concepts common to all restoration projects in the CRE. The guidelines entail types of restoration projects, project selection guidelines, and a detailed description of the phases of a generic restoration project. Specific types of projects appropriate for each of the five main restoration strategies are identified along with expected ecosystem benefits (Table 3.1). The proposed selection guidelines enhance existing guidelines by intensifying their ecosystem basis. Restoration project phases are summarized in a checklist (Table 3.5) that project sponsors might use to organize their efforts. The five main project phases are planning, implementation, effectiveness monitoring, data management and dissemination, and adaptive management. The implementation guidelines apply the restoration science established in Section 2 (Scientific Basis) to on-the-ground projects, so that the likelihood of success is maximized. 


\subsection{Discussion and Recommendations}

This document was developed to assist the BPA and the COE to address the habitat needs of listedsalmonids in the Columbia River estuary. The study area is defined as the area from Bonneville Dam to the river mouth. It provides guidelines for the decision-making process from project conceptualization to implementation to post-completion monitoring and follow up. It does not provide all the answers nor is it a step-by-step formula that is guaranteed to result in successful restoration. It is meant to guide planners, project sponsors, government agencies, and interested parties through a thoughtful, scientific process that will result in well developed, ecosystem-based restoration projects that have a good chance to succeed based on our current understanding of the habitat needs of juvenile salmonids. This document is a workin-progress and will allow for adaptive management as projects are implemented and monitored. Interested stakeholders should study the approach and apply the CRE restoration strategies and implementation guidelines to their particular planned or ongoing projects. Then feedback and comments on the approach should be provided so that it can be revised and improved.

This habitat restoration approach is one piece of a coordinated suite of actions being undertaken to improve salmonid survival and productivity in the CRE ecosystem. Other elements include: 1) a broadscale action plan (RPA Action 158) that will serve as an umbrella document for COE and BPA actions in the estuary to implement the FCRPS biological opinion; 2) an overall research, monitoring, and evaluation plan (RPA Action 161) designed specifically for the CRE; 3) a revised conceptual CRE ecosystem model (RPA Action 162) that will contribute to the scientific foundation for the actions; 4) research to investigate the fundamental biological and physical linkages within various habitat types to better understand the habitat requirements of juvenile salmonids in the CRE (RPA Action 196); 5) subbasin planning (RPA Action 154); and 6) numerous on-the-ground restoration projects (RPA Action 160). These and other actions and authorities comprise the Action Agencies' program for the CRE.

The relationship between habitat restoration planning and research, monitoring, and evaluation is especially important. The RME plan for the estuary and plume currently under development by the Action Agencies identified the following uncertainties in the knowledge base (BPA et al. 2003): habitat usage by juvenile salmon in the tidal freshwater reach of the CRE (RM 46-146); the linkage between habitat type and survival; spatial and temporal usage of CRE habitats by listed salmon with diverse life histories; accessibility of habitat to juvenile salmon; and attributes of the CRE that are limiting for the listed salmon populations. Resolving these uncertainties, which are consistent with those listed in Section 2.3.6, will have a direct bearing on restoration planning. Furthermore, the RME plan offers an approach for effectiveness monitoring of restoration actions at both the project and program levels (BPA et al. 2003). Research and monitoring results will be evaluated and applied to restoration planning, i.e., adaptive management will be used.

This document emphasizes the need for extensive coordination, collaboration, and planning. A framework is developed (see 3.0 Implementation Guidelines) within which planning, implementation, monitoring, and adaptive management should occur. The document identifies the importance of carefully evaluating each potential action on its own merits, its relationship to the larger ecosystem, and how it links to other actions occurring both inside and outside the CRE. It also emphasizes that each action must be viewed as an experiment that will help provide a better understanding of ecosystem processes, reduce critical uncertainties in our knowledge of salmonid habitat needs, and enhance our ability to implement successful restoration efforts. 


\subsection{Caveats and Constraints}

There are several important caveats and constraints on this CRE habitat restoration approach. By explaining these, the purpose is to improve understanding of the context in which this approach will be applied. The more these constraints are understood, the more the implementation process can progress in a collaborative, knowledgeable manner.

1. Ecosystem vs. Habitat Restoration -- Restoration of an entire ecosystem is not generally practical. Individual habitat restoration projects, however, have the greatest likelihood of success when they are implemented with an ecosystem perspective, i.e., they are ecosystem-based. This is why the document's development progresses from the scientific basis at the ecosystem level to implementation at the project level. Accordingly, the document's goal and principles address habitat restoration projects using an ecosystem context.

2. Funding Limitations -- The funding base is limited despite its recent growth. The finite amount of money for restoration makes it imperative that the process for project selection, monitoring, and adaptive management is clear and robust so that funds are expended as cost-effectively as possible.

3. Federal Institutional Differences -- The three main federal agencies funding CRE habitat restoration are the BPA, the COE, and the EPA. These agencies have different missions, operating authorities, and funding rules affecting which projects they can and cannot fund. When cost-sharing is required under $\mathrm{COE}$ restoration authorities, BPA is authorized to provide the local share, meaning these agencies must work together toward a common end.

4. COE Authorities -- The COE's authorities pertaining to restoration (see Appendix A for a detailed explanation) have specific rules and conditions that can affect project implementation. For example, WRDA Section 536 projects must have a 35\% state/local cost-share element, the COE does not have nor is likely to receive condemnation authority, and monitoring and adaptive management are usually limited to $1 \%$ and $3 \%$, respectively.

5. Federal, State, and Local Laws - Implementation of restoration projects is subject to various federal and state laws such as the Endangered Species Act, the Clean Water Act, and the Coastal Zone Management Act (CZMA). Specific goals within these laws may preclude certain habitat development activities. As part of the CZMA consistency determination, county comprehensive plans are reviewed; these local land use guidelines may place limitations on habitat restoration actions. For example, the Clatsop County Comprehensive Plan prohibits certain actions within a historic fishing area.

6. Habitat Availability - The amount of habitat available for restoration is limited. Habitat mapping and inventory work are underway to quantify the existing habitat types. Generally, it should be recognized that the finite amount of habitat available for restoration will constrain restoration efforts. For example, the 10,000-acre goal for habitat restoration in RPA Action 160 represents $44 \%$ of the available land below RM 46 (T. Berquam, COE Portland District, pers. comm., September 16, 2003). 
7. Land Ownership - In the CRE, most land is privately owned. As a result, habitat restoration planning will have to consider the social, political, and community ramifications that changes in land use practices could produce.

8. Other Restoration Initiatives: One of the purposes of this document is to provide a framework within which all parties involved with restoration work in the CRE can work collaboratively. It has no authority and thus is only as good as individual organizations' willingness to participate within this framework.

9. Knowledge Base: As noted throughout the text, there are gaps in our knowledge base that limit our ability to know exactly what restoration actions will provide the best possible outcomes for salmonids. All users of this document must bear that in mind and be prepared to adjust strategies as implementation progresses and to share lessons learned freely with all interested parties.

10. Outcome Uncertainties - The level of uncertainty constrains our ability to accurately predict the outcome of actions. Ecosystem restoration is a developing field, and each project will have some level of uncertainty. Restoration in the CRE represents an opportunity to reduce uncertainties and learn from each project, although each project and the program as a whole should have target outcomes.

11. Multiple Influences -- The mainstem Columbia River and tributaries, the estuary below Bonneville Dam, the plume, and the Pacific Ocean form a continuous, interacting series of habitats for juvenile and adult salmonids. Events or regimes in one area can affect other areas. For example, zooplankton prey resources in the estuary will differ between El Nino and La Nina oceanic regimes (Peterson 1999), possibly affecting growth rates for some juvenile salmon. At the other end, FCRPS operations affect river discharge into the estuary and plume, affecting water surface elevations, the location of the mixing zone, and other phenomena in the CRE. Thus, influences on CRE ecosystem structure and function from the Columbia River upstream and the ocean downstream must be considered, although not necessarily controlled, during CRE habitat restoration.

\subsection{Next Steps}

This document establishes a scientific basis and implementation guidelines for habitat restoration projects in the Columbia River estuary. The goal of the CRE habitat restoration program is "implementation of well coordinated, scientifically sound projects designed to enhance, protect, conserve, restore, and create 10,00 acres tidal wetlands and other key habitats to aid rebuilding of ESA-listed salmon populations and native species using the Columbia River estuary." Suggested next steps ${ }^{1}$ to help fulfill this goal are as follows.

Coordinating Body -- Organize and empower a coordinating body for habitat restoration activities in the CRE.

To effectively implement this approach, the stakeholders should institute a coordinating body. The purpose of this body would be to provide technical and policy oversight of habitat restoration

\footnotetext{
${ }^{1}$ The discussion of next steps incorporates review comments provided by J. Marshall (2003), the Independent Scientific Review Panel (2003), and NOAA Fisheries (2003).
} 
activities in the CRE. The coordinating body would also act as a forum for bringing stakeholders together to evaluate proposed projects, seek support and funding for projects, discuss progress and new developments, review monitoring and restoration activities, assess project effectiveness, share data, and disseminate information. It is quite possible that this body could be developed from an existing forum(s), such as the Estuary Partnership.

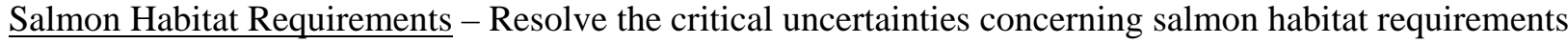
in the CRE.

To reiterate, these uncertainties (see Juvenile Salmon Usage) include research to: understand the relationship between life history strategies and habitat requirements; juvenile usage and basic ecology of the tidal freshwater (RM 46-146) subsystem; linkages between biological and physical processes in various habitat types; the landscape qualities of preferred habitats; growth and survival rates for various life history types in relation to habitat attributes; habitat forming processes for key habitat types; and food web interactions. We need to know much more about specific habitat requirements for juvenile salmonids in the CRE to implement effective habitat restoration projects. Pertinent research is underway and should be continued with technical oversight and coordination with habitat restoration managers to assure that results can be applied to CRE habitat restoration efforts. Restoration projects, however, will likely be implemented despite the knowledge gaps.

Habitat Mapping and Inventory - Intensify the ongoing habitat mapping effort, and apply the results to develop a comprehensive, quantified habitat inventory for the CRE.

Habitat mapping and inventory are critical steps in restoration planning. Such work is underway and is producing useful results, but much remains to be done. For example, all diked lands and their elevations should be inventoried, and the relative degree of disturbance cataloged to provide a first step to identify shallow-water areas with possible restoration potential. In addition, high resolution habitat maps from the Estuary Partnership are scheduled for release soon. They should be studied, integrated with data on salmon habitat requirements, and the collective results applied to the restoration planning process. The result of this integration would be a map of those habitats that have the highest potential to be successfully restored while offering the greatest potential benefits to salmon.

Selection Guidelines and Project List - Finalize the proposed project selection guidelines and list specific restoration projects and potential sites.

A new set of project selection guidelines is proposed in this document. The new guidelines build on previous work with enhancements related to the ecosystem basis for the project. Any coordinating body that materializes will need to examine the project selection guidelines herein and consider further refinement. For example, the Coordinating Body could support the development of functional assessment methods that better characterize and calibrate the ecological functions of degraded or converted historical habitat types from existing reference sites. This will help frame the tracking of the potential ecological benefit to be gained from applying a restoration strategy. The results could then be incorporated into a prioritized list of projects for the decision making process. Data for each potential project on the list should include acreage, location, owner, type of project, proposed restoration strategy, and results of the project selection analysis.

Project Scale -- Prioritize restoration projects that promote the long-term sustainability of ecosystem function and structure at the landscape scale wherever possible. 
Small-scale, short-term projects selected on a site-by-site basis certainly have their role. However, large-scale projects may be preferable to small-scale projects, although many other factors should be considered, such as cost.

Project Credit - Consider changing from RM 0-46 to RM 0-146 the credit given for projects under BiOp RPA Action 160.

Action 160 calls for 10,000 acres of habitat restoration in the estuary (RM 0-46). This causes a focus of habitat restoration efforts on the estuary, which is definitely worthwhile, but is possibly at the expense of the tidal freshwater reach RM 46-146. In this document, we treated the entire tidally influenced portion of the Columbia River and estuary as a whole for the purpose of habitat restoration. A similar approach might be considered if the federal parties revisit the BiOp RPA actions.

Project Trust Fund - Establish a trust fund for CRE habitat restoration projects.

Appropriation and funding cycles do not always coincide with when sellers of property for restoration are ready to sell. If a trust fund were available, opportunities for worthwhile projects might not be lost. A trust fund would also stabilize the implementation process, i.e., allow progress in a more orderly manner than would be the case without it and help ensure adequate monitoring.

Monitoring - Perform effectiveness monitoring for implemented projects and develop methods to evaluate cumulative effects of multiple projects.

Monitoring is necessary to measure success and should be a part of most, if not all, projects. Resources should be directed to support the capacity of researchers and restoration project managers toward a coordinated effort that standardizes monitoring methods, thereby contributing to rigorous comparisons of results stemming from restoration projects in the CRE. On a collective basis, multiple projects may have cumulative effects not observable at the local scale. Methods, however, need to be developed to assess cumulative effects.

Data - Establish a data system for CRE habitat restoration.

Data management and dissemination are critical elements of the CRE habitat restoration activities. Currently no universally used system exists. Therefore the funding agencies will consider development of a comprehensive, interactive data management system that would house, store, analyze, and disseminate data on the CRE restoration projects and other relevant data.

Adaptive Management - Embrace a true adaptive management process as part of the CRE habitat restoration efforts.

Restoration actions should provide new insight into the functioning of the CRE ecosystem. In addition, results from research on juvenile salmon usage in the CRE should be applied as they become available. This, in turn, should enhance our ability to predictably restore habitats. As a corollary to this, restoration projects should be treated as "learning opportunities." This perspective will cause implementers to recognize possible uncertainty in the outcome and design the project and associated post-construction monitoring accordingly. An adaptive management perspective is particularly important in the CRE because outcomes may be unpredictable given that our understanding of the salmonid habitat requirements is limited. 
Collaboration - Collaborate to implement CRE habitat restoration projects.

CRE habitat restoration projects by their very nature will require extensive collaboration among often diverse stakeholders. For example, COE restoration funding is often contingent upon a state/local cost share agreement, necessitating collaborative efforts to institute. Collaboration also means that the stakeholders seriously engage in the process. In addition, project sponsors are often at the heart of the debate between the ecological significance of a proposed restoration site and possible impacts to the surrounding local community. Collaboration is essential for the coordinating body recommended above and for restoration, in general, to succeed.

Follow-Up Work - Apply this approach and, as new information becomes available, institute follow-up work on a project implementation guidebook and a revised strategic plan for CRE habitat restoration.

We encourage the stakeholders to use this approach and revise it as necessary to ensure its continued viability and usefulness. As restoration work progresses, it may be useful to follow this document with a detailed, CRE-specific "guidebook" for restoration project implementation. Finally, while this document provides general recommendations for restoration strategies by subarea, it needs to be revised to form an action plan that identifies specifically "... what restoration actions should be done, where they should be done, and when they should be done" (NOAA Fisheries 2003). A habitat restoration action plan is needed to help put each project into context.

In conclusion, this document provides a scientific basis and implementation guidelines for a habitat restoration program designed to improve ecosystem functions and enhance juvenile salmonid survival in the CRE. As more experience is gained with CRE habitat restoration and scientific uncertainties are resolved, this document should be used as a basis for a detailed habitat restoration plan that specifically addresses 1) which habitat types offer the greatest ecological benefit to salmon, 2) the location of potential sites that if restored would likely provide these habitat types, and 3) how and when the restoration work should be done. This document supports the use of adaptive management so that all elements of salmonid habitat restoration actions in the CRE are under continual evaluation and revision at both the project and program levels. Lessons learned from current and proposed habitat restoration projects need to be applied to all future work, such as the Estuary Partnership's habitat restoration program and the Corps of Engineers' General Investigation Study for the CRE, to ensure the most effective use of resources and the best possible long term environment for salmonid growth and survival in the CRE. 


\subsection{Literature Cited}

Bisson, P. and nine co-authors. 2000. The Columbia River Estuary and the Columbia River Basin Fish and Wildlife Program. Independent Scientific Advisory Board, Portland, Oregon, ISAB 2000-5.

Bonneville Power Administration, U.S. Army Corps of Engineers, Portland District, and Pacific Northwest National Laboratory. 2003. Research, monitoring, and evaluation plan for the Columbia River estuary and plume. Report submitted to NOAA Fisheries. Portland, Oregon. September 30, 2003.

Bottom, D. L. and K. K. Jones. 1990. Species composition, distribution, and invertebrate prey of fish assemblages in the Columbia River estuary. Prog. Oceanogr. 25: 243-270.

Bottom, D. L., C. A. Simenstad, A. M. Baptista, D. A. Jay, J. Burke, K. K. Jones, E. Casillas, and M. H. Schiewe. 2001. Salmon at River's End: The role of the estuary in the decline and recovery of Columbia salmon. National Marine Fisheries Service, National Oceanic and Atmospheric Administration, Seattle, Washington.

Bottom, D., K. Jones, and M. Herring. 1984. Fishes of the Columbia River estuary. Final report on the fish work unit of CREDDP. Corvallis, Oregon.

Brett, J. R. 1971. Energetic Responses of Salmon to Temperature: A Study of Some Thermal Relations in the Physiology and Freshwater Ecology of Sockeye Salmon (Oncorhynchus nerka). American Zoology 11(1): 99-113.

Brinson, M. M. 1993. A Hydrogeomorphic Classification for Wetlands. Wetlands Research Program Technical Report WRP-DE-4. U.S. Army Corps of Engineers, Waterways Experiment Station, Vicksburg, Mississippi.

Bureau of Reclamation, U.S. Army Corps of Engineers, and Bonneville Power Administration. 2002. Final Endangered Species Act 2003/2003-2007 Implementation Plan for the Federal Columbia River Power System. Portland, Oregon. October 2002.

Busby, P. J., T. C. Wainwright, G. J. Bryant, L. J. Lierheimer, S. Waples, F. W. Waknitz, and I. V. Lagomarsino. 1996. Status review of west coast steelhead from Washington, Idaho, Oregon, and California: National Oceanic and Atmospheric Administration, Technical Memorandum; NMFSNWFSC-27.

Busch, D. E. and J. C. Trexler. 2003. Monitoring Ecosystems: Interdisciplinary Approaches for Evaluating Ecoregional Initiatives. Washington, DC: Island Press.

Cairns, J. Jr. 1988. Restoration and the alternative: a research strategy. Restoration and Management Notes 6: 65-67.

Cairns, J. Jr. 1989. Restoring damaged ecosystems: is predisturbance condition a viable option? Environ. Prof. 11: 152. 
Cairns, J. Jr. 1991. The status of the theoretical and applied science of restoration ecology. Environ. Prof. 13: 186-194.

Clark, C., and J.E. Shelbourn. 1985. Growth and Development of Seawater Adaptability by Juvenile Fall Chinook Salmon (Oncorhynchus tshawytscha) in Relation to Temperature. Aquaculture 45:21-31.

COE, American Rivers, CREST, LCREP. 2001. Lower Columbia River and Estuary Habitat Conservation and Restoration Workshop Proceedings. Portland, Oregon.

COE. 1996. Lower Columbia River Bi-State Water Quality Program Fish, Wildlife and Wetlands GIS Mapping. USACE Portland District, Geotechnical Engineering Branch, GIS, Survey, and Mapping Section. Report submitted to the Bi-State Water Quality Program. Portland, Oregon.

COE. 2001. Biological Assessment Columbia River Channel Improvements Project. Submitted to NMFS and USFWS. December 28, 2001. Portland, Oregon.

Corps of Engineers. 2000. Planning Guidance Notebook. Washington, DC. ER 1105-2-100.

Craddock, D. R., T. H. Blahm, and W. D. Parente. 1976. Occurrence and utilization of zooplankton by juvenile Chinook salmon in the lower Columbia River. Trans. Amer. Fish. Soc. 1: 72-76.

Dawley, E., R. Ledgerwood, T. Blahm, C. Sims, J. Durkin, R. Kirn, A. Rankis, G. Monan, and F. Ossiander. 1986. Migrational characteristics, biological observations, and relative survival of juvenile salmonids entering the Columbia River estuary, 1966-1983. Final report by the Northwest and Alaska Fisheries Center, NOAA to the Bonneville Power Administration. Portland, Oregon.

Diefenderfer, H.L., R.M. Thom, and J.E. Adkins. 2003. Systematic Approach to Coastal Ecosystem Restoration (draft). PNWD-3237, prepared for National Oceanic and Atmospheric Administration Coastal Services Center, by Battelle, Richland, Washington.

Durkin, J. T. 1982. Migration characteristics of Coho salmon (Oncorhynchus kisutch) in the Columbia River and its estuary. In: Estuarine Comparisons, V. Kennedy (ed.). Academic Press, New York.

Earn, D. J., S. A. Levin, and P. Rohani. 2000. Coherence and conservation. Science 290: 1360-1364.

Fisher, J. P. and W. G. Pearcy. 1996. Dietary overlap of juvenile fall- and spring-run Chinook salmon, Oncorhynchus tshawytscha, in Coos Bay, Oregon. Fisheries Bulletin 95: 25-38.

Forman, R. T. T. and M. Godron. 1986. Landscape Ecology. John Wiley and Sons.

Fresh, K. and eleven co-authors. 2003. Guidance for protection and restoration of the nearshore ecosystems of Puget Sound. Draft report prepared by the Nearshore Science Team in support of the Puget Sound Nearshore Ecosystem Restoration Program. Seattle, Washington. May 2, 2003.

Garano, R., R. Robinson, and C. Simenstad. 2002. Estuarine landcover along the Columbia River estuary determined from Landstat 7 ETM+ imagery. Report submitted to the Lower Columbia River Estuary Partnership. Portland, Oregon. 
Gentile, J. H., M. A. Harwell, W. Cropper Jr., C. C. Harwell, D. DeAngelis, S. Davis, J. C. Ogden, and D. Linman. 2001. Ecological conceptual models: a framework and case study on ecosystem management for South Florida sustainability. The Science of the Total Environment 274: 231253.

Gonzales, A., J. H. Lawton, P. S. Gilbert, T. M. Blackburn, and J. Evans-Freke. 1998. Meta population dynamics, abundance, and distribution on a micro-ecosystem. Science 281: 2045-2047.

Graves, J. K., J. A. Christy, P. J. Clinton, and P. L. Britz. 1995. Historic Habitats of the Lower Columbia River. Report by the Columbia River Estuary Study Taskforce, Astoria, Oregon.

Greene, C. T., T. Beeclie, and M. Ruckelshaus. 2003. Linking-habitat-related and density-dependent population responses in Chinook salmon. Abstract. Georgia Basin/Puget Sound Research Conference.

Gustafson, R. G., T. C Wainwright, G. A Winans, F. W. Waknitz, L. T Parker, and R. S Waples. 1997. Status review of sockeye salmon from Washington and Oregon: National Oceanic and Atmospheric Administration, NOAA Technical Memorandum NMFS-NWFSC-33.

Hard, J. J., R. G. Kope, W. S. Grant, W. Waknitz, L. T Parker, and R. S. Waples. 1996. Status review of pink salmon from Washington, Oregon, and California: U.S. Department of Commerce, National Oceanic and Atmospheric Administration. NOAA Technical Memorandum NMFS-NWFSC-25.

Hobbs, R. J., and D. A. Norton. 1996. Towards a conceptual framework for restoration ecology. Restoration Ecology 4: 93-110.

Horner, R. R. and K. J. Raedeke. 1989. Guide for wetland mitigation project monitoring. Washington State Department of Transportation, Olympia, Washington. Report No. WA-RD 195.1.

Huggett, R. J. 1993. Modeling the Human Impact on Nature. Oxford University Press.

Hughes, J. E., L. A. Deegan, M. J. Weaver, and J. E. Costa. 2002. Regional application of an index of biotic integrity based on fish communities. Estuaries 25: 250-263.

Independent Scientific Review Panel. 2003. Review of the draft: "An ecosystem-based restoration plan with emphasis on salmonid habitats in the Columbia River estuary." Report for the Northwest Power and Conservation Council. Portland, Oregon. September 8, 2003. ISRP 2003-13.

Independent Scientific Group. 2000. Return to the River 2000: Restoration of salmonid fishes in the Columbia River ecosystem. Report submitted to the Northwest Power Planning Council. Portland, Oregon. Document NWPPC 2000-12.

Johnsen, R. C. and C. W. Sims. 1973. Purse seining for juvenile salmon and trout in the Columbia River estuary. Transactions American Fisheries Society 102: 341-345.

Johnson, O. W., M. H. Ruckelshaus, W. S. Grant, F. W. Waknitz, A. M. Garrett, G. J. Bryant, K. Neely, and J. J. Hard. 1999. Status review of coastal cutthroat trout from Washington, Oregon, and California: National Oceanic and Atmospheric Administration; NOAA Technical Memorandum NMFS-NWFSC-37. 
Johnson, O. W., W. S. Grant, R. G. Kope, K. Neely, F. W. Waknitz, and R. S. Waples. 1997. Status review of chum salmon from Washington, Oregon, and California: National Oceanic and Atmospheric Administration. NOAA Technical Memorandum. NMFS-NWFSC-32.

Jordan, F., H. L. Jelks, and W. M. Kitchens. 1997. Habitat structure and plant community composition in a northern Everglades wetland landscape. Wetlands 17(2): 275-283.

Kareiva, P., M. Marveir, and M. McClure. 2000. Recovery and management options for spring/summer Chinook salmon in the Columbia River basin. Science 290: 977-979.

Karr, J. R. 1993. Measuring biological integrity: lessons from streams. In: Ecological Integrity and the Management of Ecosystems, eds. S. Woodley, J. Kay, and G. Francis, pp. 83-104. Sponsored by Heritage Resource Centre, University of Waterloo, and Canadian Parks Service, Ottawa, Canada.

Kentula, M. E. 1997. A step toward a landscape approach in riparian restoration. Restoration Ecology 5: $2-3$.

Lee, R. M. and J. N. Rinne. 1980. Critical thermal maxima of five trout species in the Southwestern United States. Transactions of the American Fisheries Society 109:632-635.

Lewis, R. R., III. 1990. Wetland restoration/creation/enhancement terminology: suggestions for standardization. Pages 417-419 in Jon A. Kusler and Mary E. Kentula (eds). Wetland creation and restoration the status of the science. Island Press, Washington, D. C.

Lower Columbia River Estuary Partnership. 2003. Work plan for a subbasin plan for the Mainstem Lower Columbia River and Columbia River Estuary Subbasins. Prepared for the Northwest Power Planning Council. Portland, Oregon. January 2003.

Lower Columbia River Estuary Program. 1998. Lower Columbia River Estuary Plan, Aquatic Ecosystem Monitoring Strategy for the Lower Columbia River and Information Management Strategy. Volume II. Portland, Oregon.

Lower Columbia River Estuary Program. 1999. Lower Columbia River Estuary Plan, Comprehensive Conservation and Management Plan. Volume I, Portland, Oregon.

Marriott, D. and 25 co-contributors. 2001. Lower Columbia River and Columbia River Estuary Subbasin Summary. Draft submitted to the Northwest Power Planning Council. Portland, Oregon. October 26, 2001.

Marshall, J. 2003. Comments on long term monitoring strategy/An ecosystem-based restoration plan with emphasis on salmonid habitats in the Columbia River estuary. Electronic mail to the Estuary Partnership. July 22, 2003.

Marshall, J., D. Fox, M. Barnes, and H. West. 1987. Mitigation and restoration plan for the Columbia River Estuary. Report by the Columbia River Estuary Study Taskforce. Astoria, Oregon.

McCabe, G. T., R. L. Emmett, W. D. Muir, and T. H. Blahm. 1986. Utilization of the Columbia River estuary by subyearling Chinook salmon. Northwest Science 60: 113-124.

McCabe, G. T., W. D. Muir, R. L. Emmett, and J. T. Durkin. 1983. Interrelationships between juvenile salmonids and nonsalmonid fish in the Columbia River estuary. Fish. Bull. 81: 815-826. 
McIntire, C. D. and J. A. Colby. 1978. A hierarchical model of lotic ecosystems. Ecological Monographs 48:167-190.

Meffe, G. K., C. R. Carroll, and contributors. 1994. Principles of Conservation Biology. Sinauer Assoc., Inc., Sunderland, Mass.

Morgan, J., L. Marston, and R. Holton. 1979. An annotated bibliography of the Columbia River estuary. Final report by the Oregon Estuarine Research Council. Corvallis, Oregon. Sea Grant Publication ORESU-L-79-002.

National Marine Fisheries Service. 2000. Reinitiation of consultation on operation of the Federal Columbia River Power System, including the juvenile fish transportation program, and 19 Bureau of Reclamation projects in the Columbia Basin. Biological Opinion. National Marine Fisheries Service, Northwest Region, Seattle, Washington.

National Marine Fisheries Service. 2003. Viability Criteria for Willamette and Lower Columbia Basin Pacific Salmonids. Interim Report by the Willamette/Lower Columbia Technical Recovery Team. ed. Portland, Oregon; 2003 Mar 31.

National Research Council. 1992. Restoration of Aquatic Ecosystems. National Academy Press, Washington, D.C.

National Research Council. 2001. Compensating for Wetland Losses Under the Clean Water Act. National Academy Press, Washington, D.C.

Neal, V. T. 1972. Physical aspects of the Columbia River and its estuary. In: The Columbia River Estuary and Adjacent Ocean Waters, A. T. Pruter and D. L. Alverson (eds.), pp. 19-40. University of Washington Press, Seattle, Washington.

NOAA Fisheries. 2003. Plan 159 - Comments. Provided by C. Tortorici (NOAA Fisheries) to G. Johnson (PNNL). September 3, 2003.

Noggle, C. C. 1978. Behavioral, physiological and lethal effects of suspended sediment on juvenile salmonids. Thesis, University of Washington. Seattle, Washington.

Northwest Power Planning Council. 2000. 2000 Columbia Basin Fish and Wildlife Program. Portland, Oregon. Pub. No. 2000-19.

Odum, H. T. 1988. Self-Organization, Transformity, and Information. Science 242:1132-1139.

Odum, H. T. and D. A. Hornbeck. 1997. EMERGY evaluation of Florida salt marsh and its contribution to economic wealth. In Ecology and Management of Tidal Marshes: A Model from the Gulf of Mexico. Charles L. Coultas and Yuch-Ping Hsieh, (eds). St. Lucie.

Ogden, J. C., S. M. Davis, and L. A. Brandt. 2003. Science strategy for a regional monitoring and assessment program: the Florida Everglades example. Pages 135-163, in: Busch, D.E. and Trexler, J.C. (eds), Monitoring Ecosystems. Island Press, Washington, D. C.

Peterson, W. T. 1999. Hydrography and zooplankton off the Central Oregon coast during the 1997-1998 El Nino Event. Proceedings of the 1998 Science Board Symposium on the Impacts of the 1997/1998 El Nino Event on the North Pacific Ocean and its Marginal Seas. PICES Scientific Report No. 10. 
Proctor, C. M., J. C. Garcia, D. V. Galvin, T. Joyner, G. B. Lewis, L. C. Loehr, and A. M. Massa. 1980. An ecological characterization of the Pacific Northwest coastal region. Volume one: conceptual model: Biological Services Program, U.S. Fish and Wildlife Service, FWS/OBS-79/11.

Raynie, R. C. and J. M. Visser. 2002. CWPPRA adaptive management review final report. Prepared for the CWPPRA Planning and Evaluation Subcommittee, Technical Committee, and Task Force, Coastal Restoration Division, Louisiana Department of Natural Resources.

Restore America's Estuaries and NOAA. 2003. A national strategy to restore coastal and estuarine habitat. Executive summary. Arlington, Virginia.

Rosenzweig, M. L. 1987. Restoration ecology: a tool to study population interactions? In: W.R. Jordan I.I.I., M. E. Gilpin, and J. D. Aber (eds.) Restoration Ecology: A Synthetic Approach to Ecological Research, pp. 189-204. Cambridge University Press, New York, New York.

Shafer, D. J. and D. J. Yozzo. 1998. National guidebook for application of hydrogeomorphic assessment to tidal fringe wetlands. Wetlands Research Program Technical Report, U.S. Army Corps of Engineers, Waterways Experiment Station, Vicksburg, Mississippi. WRP-DE-16.

Sherwood, C., D. A. Jay, R. B. Harvey, P. Hamilton, and C. A. Simenstad. 1990. Historical Changes in the Columbia River Estuary. Progress in Oceanography 25: 299-352.

Short, F. T. 2003. Interactive GIS-based site selection model for eelgrass restoration on a CD-ROM. http://ciceet.unh.edu/abstract.php?fullprojid=95. March 13, 2003

Shreffler, D. K. and R. M. Thom. 1993. Restoration of urban estuaries: New approaches for site location and design by: Washington Department of Natural Resources: Battelle Pacific Northwest Laboratories; 1993107.

Simenstad, C. A. 1983. The ecology of estuarine channels of the Pacific Northwest coast: a community profile. U.S. Fish and Wildlife Service. Olympia, Washington. FWS/OBS-83/05.

Simenstad, C. A. and J. R Cordell. 2000. Ecological assessment criteria for restoring anadromous salmonid habitat in Pacific Northwest estuaries. Ecological Engineering 15:283-302.

Simenstad, C. A. and R. M. Thom. 1992. Restoring wetland habitats in urbanized Pacific Northwest estuaries. Thayer, G. W. Restoring the Nation's environment. College Park, Maryland: Maryland Sea Grant; 1992; pp. 423-472.

Simenstad, C. A., K. L., Fresh, J. Flemma, and D. Clarke. 1991. Effects of estuarine habitat modifications on anadromous salmonids: A literature survey. Seattle, Washington: Wetland Ecosystem Team, Fisheries Research Institute, School of Fisheries, University of Washington; 1991; FRI-UW-9123.

Simenstad, C., D. Jay, C. McIntire, W. Nehlsen, C. Sherwood, and L. Small. 1984. The dynamics of the Columbia River ecosystem. Final report of the Columbia River Estuary Data Development Program.

Smith, D. W. 1978. Tolerance of juvenile chum salmon (Oncorhynchus keta) to suspended sediments. Thesis, University of Washington, Seattle. $86 \mathrm{pp}$. 
Store, R. and J. Kangas. 2001. Integrating spatial multi-criteria evaluation and expert knowledge for GIS-based habitat sustainability modeling. Landscape and Urban Planning 55: 79-93.

TetraTech. 1996. Lower Columbia River Bi-State Program: The Health of the River 1990-1996: Integrated Technical Report. Report submitted to the BiState Water Quality Program. Portland, Oregon.

Thom, R. M. 1997. System-development matrix for adaptive management of coastal ecosystem restoration projects. Ecological Engineering 8: 19-232.

Thom, R. M. 2000. Adaptive management of coastal ecosystem restoration projects. Ecological Engineering 15(3-4): 365-372.

Thom, R. M. and K. F. Wellman. 1996. Planning aquatic ecosystem restoration monitoring programs. Institute for Water Resources, U. S. Army Corps of Engineers Alexandria Virginia and Waterways Experimental Station U. S. Army Corps of Engineers Vicksburg Mississippi, IWR Report 96-R-23.

Thom, R. M., H. L. Diefenderfer, and K.D. Hofseth. In Press. A framework for risk analysis in environmental investments: The U.S. Army Corps of Engineers restoration project planning process. In Bruins, R. and Heberling, M. (eds.) Economics and ecological risk assessment: Applications to watershed management. Boca Raton, Florida: CRC Press.

Thomas, D. W. 1983. Changes in the Columbia River estuary habitat types over the past century. Columbia River Estuary Data Development Program, Columbia River Estuary Task Force, Astoria, Oregon.

Thorpe, J. E. 1994. Salmonid fishes in the estuarine environment. Estuaries 17: 76-93.

Tiffan, K. F., D. W. Rondorf, and P. G. Wagner. 2000. Physiological development and migratory behavior of subyearling fall Chinook salmon in the Columbia River. North American Journal of Fisheries Management 20: 28-40.

USFWS. 1998. Department of Interior. Vol. 3: Characterization Atlas, Zone and Habitat.

Wagner, H. H., F. P. Conte, and J. L. Fessler. 1969. Development of osmotic and ionic regulation in two races of Chinook salmon Oncorhynchus tshawytscha. Comparative Biochemistry and Physiology 29: $325-341$.

Washington State Department of Fish and Wildlife. 1992. Salmon and Steelhead Stock Inventory (SaSI), "Bluebook." Olympia, Washington.

Washington State Department of Fish and Wildlife. 2000. Salmonid Stock Inventory (SaSI), coastal cutthroat trout volume. Olympia, Washington.

Weins, J. A. 1985. Vertebrate responses to environmental patchiness in arid and semiarid ecosystems. Chapter 10 in Pickett, S. T. A and P. S. White, eds, The Ecology of Natural Disturbances and Patch Dynamics. Academic Press, Inc.

Weitkamp, L. A. 1993. A review of the effects of dams on the Columbia River estuarine environment, with special reference to salmonids. Draft report by the Northwest Fisheries Science Center, NOAA Fisheries submitted to the Bonneville Power Administration. Portland, Oregon. 
Weitkamp, L. A., T. C. Wainwright, G. J. Bryant, G. B. Milner, D. J. Teel, R. G. Kope, and R. S. Waples. 1995. Status review of Coho salmon from Washington, Oregon, and California: National Oceanic and Atmospheric Administration, NOAA Technical Memorandum, NMFS-NWFSC-24.

Wissmar, R. C. and C. A. Simenstad. 1998. Variability of riverine and estuarine ecosystem productivity for supporting Pacific salmon. Change in Pacific Northwest coastal ecosystems: A NOAA decision analysis series report. 


\section{Appendix A}

\section{Related Ecosystem Restoration Initiatives}




\section{Appendix A: Related Ecosystem Restoration Initiatives}

The purpose of this appendix is to place this Action Agency CRE restoration approach in the context of local and regional restoration-related initiatives. (See Section 1.1 for a national perspective.) Currently, numerous efforts involve salmon habitat restoration to varying degrees in the CRE; the regional initiatives are shown in Figure A.1. We describe how the approach will be coordinated and linked with these ongoing efforts. The approach intends to both contribute to these efforts, where applicable, as well as apply the manifestation of relevant policy initiatives as they relate to Salmon Habitat in the CRE.

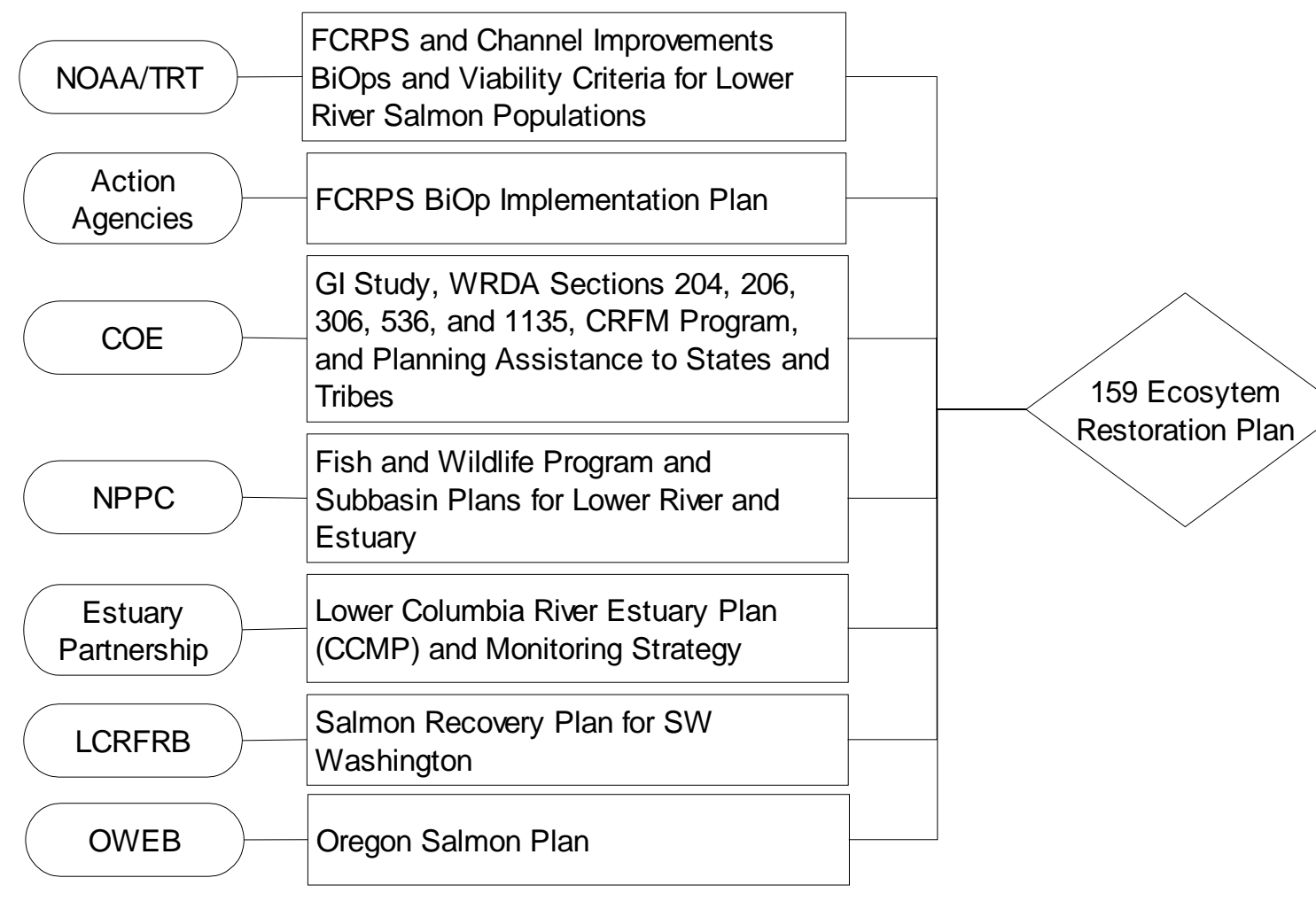

Figure A.1. Relevant regional initiatives related CRE restoration.

\section{A.1 NOAA Fisheries}

NOAA Fisheries (formerly the National Marine Fisheries Service) has statutory obligation under the ESA to render biological opinions on activities that may impact listed Pacific salmon species. In 2000, they issued a Biological Opinion on Operation of the FCRPS (NMFS 2000). This opinion contained several actions in its RPA related to the Columbia River estuary. These actions are intended to ensure the continued existence and recovery of the listed species. The action agencies for this opinion must implement the RPA. As explained earlier, this habitat restoration approach is partly in direct response to Action 159. In addition, the Plan directly relates to Action 160 calling for 10,000 acres of habitat restoration in the estuary.

NOAA Fisheries also has a statutory obligation in Section 4(f) of the ESA to develop recovery plans for all salmon and steelhead listed as threatened or endangered. Currently there are 12 evolutionarily 
significant units (ESU) of Pacific salmon and steelhead listed as threatened or endangered under the ESA that spawn and rear in the Columbia Basin (Table A.1). All of these fish spend time inhabiting the Columbia River estuary. NOAA directed technical recovery teams (TRT) to lead the development of these plans for the Columbia ESUs. The action agencies work with the TRTs, but are not obligated to fund them nor is this document (or other plans) intended to fulfill the ESA responsibilities of the TRT. The Willamette/Lower Columbia TRT has developed viability criteria for salmon populations in the lower river and estuary (NMFS 2003) that will be included in a recovery plan currently under development. This document may be used to inform the TRT process. Conversely, the document could incorporate TRT recovery measures as applicable when they are available.

Table A.1. TRTs, ESUs, and ESA listings under the FCRPS BiOp.

\begin{tabular}{lll}
\hline TRT & Evolutionarily Significant Unit & ESA Listing \\
\hline Willamette and Lower & Lower Columbia River Chinook & threatened \\
Columbia River & Upper Willamette River Chinook & threatened \\
& Columbia River Chum & threatened \\
& Lower Columbia River Steelhead & threatened \\
\hline Interior Columbia & Upper Columbia River Spring Chinook & endangered \\
River Basin & Snake River Spring/Summer Chinook & threatened \\
& Snake River Fall Chinook & threatened \\
& Upper Columbia River Steelhead & endangered \\
& Mid Columbia River Steelhead & threatened \\
& Snake River Steelhead & threatened \\
& Snake River Sockeye & endangered \\
\hline
\end{tabular}

\section{A.2 Action Agencies}

The Action Agencies for the 2000 FCRPS BiOp are the Bonneville Power Administration, the U.S. Bureau of Reclamation, and the U.S. Army Corps of Engineers. As mandated in the BiOp, they submitted an Implementation Plan (Bureau of Reclamation et al. 2002) addressing one year (2003) and five years (2003-2007). The Implementation Plan (p. 71) states "the Corps and BPA plan to continue a 10-year program to protect/enhance tidal wetlands and other key estuary habitats...the Action Agencies will support the protection and restoration of the estuary..." The action agencies identified the Plan as a key outcome of the planning effort for estuary restoration.

\section{A.3 Corps of Engineers}

In 1990, Section 306 of the Water Resources Development Act (WRDA) established environmental protection as a primary mission of the COE in planning, designing, constructing, operating, and maintaining water resource projects. This put environmental protection on an equal status with navigation and flood control. Following that change, Section 210 of WRDA 1996 established environmental protection and restoration as a primary project purpose. These mission and project purpose changes support three $\mathrm{COE}$ authorities for restoration implementation: 1) the ongoing Lower Columbia River Ecosystem Restoration General Investigation (GI); 2) the Lower Columbia Ecosystem 
Restoration Authority (WRDA Section 536); and 3) the Continuing Authorities (WRDA Sections 204, 206 and 1135). The key differences between authorities are described below.

COE restoration authorities require a non-federal sponsor to share in the costs of the projects (the cost-share requirements vary among authorities), as well as to provide operation and maintenance for the life of the project. BPA funds qualify for cost sharing.

Lower Columbia River Ecosystem Restoration General Investigation: The purpose of the ongoing GI feasibility study is to investigate and recommend appropriate solutions to accomplish ecosystem restoration in the Columbia River estuary, including wetland/riparian habitat restoration, stream and fisheries improvement, water quality, and water-related infrastructure improvements. The States of Oregon and Washington have agreed to jointly sponsor the study and have written a letter of intent dated May 1998. They are currently working through the Estuary Partnership to identify avenues to support BPA in sponsoring matched COE funds. The outcome of the GI study will be a strategic master plan for the estuary resulting in long-range, large projects. The Plan may be incorporated into or inform the GI study.

Lower Columbia Ecosystem Restoration Authority, Section 536: The primary purpose of the proposed Section 536 study is to carry out ecosystem restoration projects necessary to protect, monitor, and restore fish and wildlife habitat based in part on recommendations made by the Estuary Partnership. The outcome of Section 536 projects will be an array of small site-specific fish and wildlife restoration projects augmenting the comprehensive master plan generated by the GI study. There is no per-project limitation on this authority; however, the current program authorization is $\$ 30,000,000$ to implement the Comprehensive Conservation and Management Plans for the Columbia and Tillamook estuaries.

Continuing Authorities Sections 204, 206, and 1135: The purpose of the Continuing Authorities Program (CAP) is to save development and approval time and provide a quick response to smaller, local problems, specifically under Sections 1135 (project modifications for improvement of the environment), 206 (aquatic ecosystem restoration), and 204 (beneficial use of dredged material). Under these programs, Congress authorizes a specific dollar amount for the program and the COE has the authority to make investment decisions without specific congressional approval. The outcome of Section 204, 206, and 1135 projects will be an array of small, site-specific fish and wildlife restoration projects augmenting the comprehensive master plan generated by the GI study. The maximum per-project Federal expenditure on these programs is $\$ 5,000,000$.

Columbia River Fish Mitigation Project: The COE also has a major program that can assist in studies and research in the estuary. This program, called the Columbia River Fish Mitigation Project (CRFMP), is implemented through a regional coordination process called the Anadromous Fish Evaluation Program (AFEP). AFEP research that contributes to an understanding of the biological requirements of salmonids in the estuary may be implemented under Section 582 of WRDA 1999. AFEP provides a mechanism for the COE to identify and prioritize CRFMP funds in coordination with state, federal, and tribal fisheries managers and Action Agencies.

Planning Assistance to States and Tribes: The Planning Assistance to States and Tribes authority allows for the COE to assist Tribes, states, local governments, and other non-federal entities in the preparation of comprehensive plans for the development, utilization, and conservation of water and 
related land resources. Examples of studies that might be implemented under this authority include, but are not limited to, habitat mapping, water quality, and wetland evaluation studies.

Summary: The ongoing GI study is a comprehensive, long-range approach to address water resource problems and opportunities for the Lower Columbia River as needed. However, Sections 536, 204, 206, and 1135 are principally focused on fish and wildlife habitat. These authorities allow for immediate identification and construction of restoration projects. The GI study is intended to provide an ecosystemwide analysis of the estuary. These are parallel activities because urgency exists to initiate habitat restoration projects, especially to support salmon recovery. Ideally, a comprehensive, ecosystem-wide plan would be developed before implementing individual projects. The region, however, has supported the concurrent activities of pursuing short-term projects, while proceeding with the comprehensive studies. Overall, all three programs will be used to develop a comprehensive network of projects designed to improve the CRE's ecosystems. In conclusion, the document could be used to inform the COE's restoration efforts. The GI study, in particular, may find useful material in the Plan.

\section{A.4 Northwest Power and Conservation Council}

Amendments to the Pacific Northwest Electric Power Planning and Conservation Act of 1980 required the NPCC to "consider the impact of ocean conditions on fish and wildlife populations". This policy shift resulted in increased attention on the Columbia River estuary as an important component to consider in salmon decline and recovery. The new policy was manifested in two principles (NPCC 2000):

1. Salmon and steelhead in the Columbia River accommodate ocean mortality and environmental variability through a sufficient level of productivity and a wide range of biological diversity. Management actions and projects can restrict biological diversity and lessen the ability of salmon and steelhead to withstand environmental fluctuations.

2. The Columbia River estuary and near-shore plume are important ecological features that have been, and continue to be, negatively impacted by upriver management actions and local habitat change.

As a result, amendments were made to the NPCC's 2000 Fish and Wildlife Program targeting broad habitat strategies. The NPCC (2000) stated "The estuary is an important ecological feature that is negatively affected by upriver management actions and local habitat change...The estuary will be included as one of the planning units for this program. The freshwater plume and the ocean itself are also important habitats for salmon and are addressed in the Ocean Conditions section of this program."

In addition, the 2000 Fish and Wildlife Program advocated development of a basin-wide series of watershed-based plans for each of the 63 tributary subbasins of the Columbia River including the Columbia River estuary. Eleven subbasins were delineated in the study area for the Plan (Table A.2). The subbasin plans are to be developed by local entities, widely reviewed, and considered for adoption into the NPCC's Fish and Wildlife Program. The completed plans will help direct priorities for funding of projects under the Council's rolling provincial review process. The NPCC issued guidance to assist planners in a document called "Technical Guide for Subbasin Planners". The Technical Guide outlines important subbasin plan elements, including a subbasin assessment, an inventory of existing activities, and a management plan. 
Table A.2. Northwest Power Planning Council's provinces and subbasin designations for the subbasin planning process.

\begin{tabular}{lc}
\hline Province & Subbasins \\
\hline Columbia Estuary & Columbia Estuary*, Elochoman, Grays Bay, \\
Lower Columbia & Lower Columbia**, Cowlitz, Kalama, Lewis, Sandy, Washougal \\
\hline $\begin{array}{l}\text { *Includes tributaries to Baker Bay, Youngs Bay, and Cathlamet Bay. } \\
* * \text { Includes tributaries to Clatskanine Flats, Scappoose Creek. }\end{array}$ \\
\hline
\end{tabular}

The Plan could serve as a critical ecosystem-based resource during development of the subbasin plans for the Columbia estuary and adjacent watersheds. The RPA Plan relates to all 11 subbasin plans in the lower river and estuary in that they converge in the tidal, estuarine area. Recent additions to the Technical Guide stress the importance of addressing scales larger than what each subbasin plan addresses; hence, the Plan's ecosystem approach is applicable. In addition, linkages are being developed between the subbasin planning process and NOAA Fisheries' recovery planning needs (see below). And, the Lower Columbia Fish Recovery Board (LCFRB; see Section A.6) and the Estuary Partnership have started work to develop subbasin plans for the Columbia River estuary study and key tributary watersheds. The subbasin plans are scheduled to be completed in May 2004.

Coordination is taking place between the NOAA Fisheries and the NPCC's subbasin planners to ensure consistency and to provide mutual benefits for each initiative. NOAA Fisheries is working closely with the NPCC with the intent of using the subbasin plans as the basis for ESU-based recovery plans. However, because ESUs cover more than one subbasin, subbasin plans need to be integrated into ESUscale recovery plans (B. Lohn, NOAA Fisheries, letter to NPCC, May 2002). Conversely, interim recovery goals and related products are being developed that assist in the development of all three phases of a subbasin plan. This facilitates the utility of subbasin plans in the recovery planning process. Examples of these products include standardized assessment tools, targeted action steps for effective habitat conservation, and direction on monitoring and research methods.

This document provides information about the Columbia River estuary ecosystem that could aid subbasin and recovery planning efforts. The approach encourages subbasin planners, regional experts, and NOAA fisheries staff to incorporate the current understanding of an ecological region that is the critical thread to all of the subbasins and ESUs of the Lower Columbia Basin. The iterative nature of development of this approach encourages the incorporation of the latest methods, assessment tools, and research questions into the findings of the document, which will further our understanding of the ecosystem. This will enhance the development of targeted conservation and research actions needed for an effective restoration and monitoring program in the future.

\section{A.5 The Estuary Partnership}

In 1989, the states of Oregon and Washington, in partnership with private industry, implemented a comprehensive 5-year water quality study of the CRE. Based on the results of that study, known as the Bi-State Water Quality Study (1990-1995), the CRE was nominated and accepted into the National Estuary Program in 1995. In 1999, after a 3-year collaborative planning process, the Lower Columbia 
River Estuary Program (now known as the Lower Columbia River Estuary Partnership) completed the "Lower Columbia River Estuary Plan" for the lower 146 river miles of the Columbia River. This document identified a series of ecological and managerial problems in the lower Columbia River and provided 43 specific actions to address those problems. Foremost among the threats to the system was the loss of more than 50\% of the river's original wetland habitat. Accordingly, the Estuary Partnership targeted habitat conservation and protection as its top priority and began to address this issue following the adoption of the Estuary Plan in October 1999 by the U.S. EPA and the governors of the States of Oregon and Washington. The Plan includes two main elements related to habitat: inventory/monitoring and conservation/restoration.

Habitat Inventory and Monitoring: The Estuary Partnership developed a comprehensive ecosystem monitoring plan as part of its Lower Columbia River Estuary Plan. Entitled the Aquatic Ecosystem Monitoring Strategy for the Lower Columbia and Estuary, it provides a series of recommended monitoring actions to be phased in over time as funding and resources allow. The document includes strategies for data management, toxic contaminant and conventional pollutant monitoring, invasive species monitoring, and food web analysis. A key component of the Strategy is a detailed habitat inventory described in Section 2.4. A second important component is the implementation of a long-term habitat monitoring initiative with the goal of measuring habitat conditions over time and monitoring the effectiveness of habitat conservation/restoration projects. The inventory/monitoring effort will be used to establish baseline conditions for selected environmental attributes. Performance indicators can then be monitored over time to assess the effectiveness of restoration actions relative to performance standards. In 2003, the Estuary Partnership is scheduled to receive funding from the BPA to begin a project to develop habitat monitoring protocols, initiate ecosystem monitoring, and evaluate effectiveness of habitat projects (Estuary Province proposal 30015). A second part of the project calls for monitoring toxic contaminants to determine the effects they have on the health of the ecosystem

Habitat Conservation and Restoration Program Development: For the past three years, the Estuary Partnership has undertaken a series of collaborative projects funded primarily through grants from EPA and the State's 319 Programs. These projects were designed to establish the framework for an ecosystem restoration program and to develop a systematic, scientific approach for evaluating proposed habitat protection and restoration projects. The effort resulted in five important outcomes: 1) an inventory of habitat restoration sites, which provides a spatially referenced database on potential restoration sites in the lower river and estuary (Garono et al. 2002); 2) a network of organizations committed to the protection and restoration of the CRE including land owners, local communities, government agencies and nonprofit organizations; 3 ) a workshop on habitat conservation and restoration in the CRE, held in Astoria in June 2001 (COE et al. 2001); 4) a workshop on research needs held in Portland in March 2003; and 5) funding from BPA for ecosystem restoration projects in the CRE (Estuary Province proposals 30004 Blind Slough, 30005 Grays River, 30006 Chinook River, 30011 Channel Islands, and 30016 habitat restoration program (see http://www.cbfwf.org/files/province/estuary/projects). In addition, the Estuary Partnership has received funding from the EPA Watershed Initiative and is working with the COE to fund Section 536 projects. This document builds upon and explicitly incorporates many of the results of the Estuary Partnership's efforts related to restoration.

On an organizational level, the Estuary Partnership could act as the central coordinating body for the CRE. In this capacity, the Partnership has a board of directors with broad based representation that provides oversight and direction, and a Science Work Group with approximately 50 members that provide technical expertise and advice, which has sponsored a bi-state working group known as the ESA 
Executive Committee that provides a forum for resolving and coordinating salmon recovery-related issues. The Partnership is also the lead entity for developing the NPCC subbasin plan for the Lower Columbia and Estuary provinces. Finally the Partnership has been a key sponsor in a series of workshops designed to resolve technical issues related to ecosystem restoration.

\section{A.6 Lower Columbia Fish Recovery Board}

The Lower Columbia Fish Recovery Board (LCFRB) is a coordination body established by the State of Washington. The state's Salmon Recovery Funding Board (SRFB) designated LCFRB as the lead entity in southwest Washington to solicit, develop, prioritize, and submit habitat protection and restoration projects for SRFB consideration. As part of the recovery planning process, LCFRB has initiated a strategic watershed-based planning effort toward the recovery of salmonid species in the lower river and estuary. LCFRB distributes watershed planning funds to local areas through Watershed Resource Inventory Areas (WRIA). Each WRIA goes through several assessment phases to inventory current information in order to obtain a coarse understanding of watershed characteristics, including instream flows, water quality, and habitat conditions. Three WRIAs are currently doing watershed planning related to the Columbia River estuary. Each WRIA has a planning group representing the diverse interests of the watershed. They assist in guiding the development and assessment of products and action plans for their respective watershed. LCFRB has also led the development of a limiting factors analysis to characterize the "conditions that limit the ability of habitat to fully sustain populations of salmon." The Plan was reviewed by the LCFRB for consistency with their efforts.

\section{A.7 Oregon Watershed Enhancement Board}

The Oregon Watershed Enhancement Board is a state agency led by a policy oversight board in charge of implementing the Oregon Plan whose goal is "Restoring and protecting Oregon's watersheds through locally driven, voluntary, cooperative efforts." They promote and fund voluntary actions that strive to enhance Oregon's watersheds through several grant programs that awards more than \$20 million annually to support voluntary efforts by Oregonians seeking to create and maintain healthy watersheds. OWEB support is primarily through citizen action groups or "watershed councils." In January 2003, OWEB embarked on establishing regional priorities that will become the basis for funding decisions by the board. In the Columbia Basin, the Lower Columbia and Hood River subbasins have been selected to be a part of this process. This initiative is meant to be closely coordinated with the NPCC subbasin planning entity to identify and incorporate the priorities resulting from the subbasin planning process.

\section{A.8 U.S. Environmental Protection Agency}

The EPA administers the Clean Water Act and through that act provides base funding to the Estuary Partnership as part of the National Estuary Program. Through the CWA it also enforces water quality standards to support the beneficial uses of the nations' water bodies. In the LCRE, the EPA is the lead agency for the development of Total Maximum Daily Loads (TMDLs) for temperature, total dissolved gas and toxics to ensure that the LCRE is safe for salmonids and other native species and for human use. The EPA also provides grant monies to support wetland development projects and watershed restoration initiatives. Several of these grants have benefited the LCRE. Through its Environmental Monitoring and Assessment Program, EPA also funded a comprehensive one time sampling in 2000 of the LCRE which 
included 50 random sites. Parameters sampled included basic water quality as well as toxics in sediments and fish tissue. That data provide will provide key baseline data for future monitoring efforts.

\section{A.9 U.S. Geological Survey}

The USGS is a monitoring and research arm of the federal government. USGS maintains an ambient monitoring site on the lower Columbia River at Beaver. Basic water quality parameters and pesticides are monitored on a regular basis. Through its Biological Resources Division, the USGS also tracks the occurrence and distribution of contaminants in fish tissue and osprey eggs in the Columbia River basin and conducts specific studies on salmonids in the Columbia River basin for the COE and BPA.

\section{A.10 Other Restoration-Related Organizations}

Local organizations are conducting conservation and restoration activities in the Columbia River estuary (Table A.3). These include non-profit organizations, special districts, local governments, and other unique partnerships. Each possesses unique capacity and resources to benefit the ecological features of the study area. These resources include but are not limited to technical assistance, land acquisition expertise, site identification, planning and design, monitoring, local ecosystem knowledge, and community credibility. These groups are extremely instrumental to the success of restoration in the CRE. Given the majority of restoration opportunities reside in privately held land, groups that maintain a local presence and credibility are poised to measure the feasibility of a restoration strategy in the context of a community's local needs. Many of these groups participate in the Estuary Partnership's Science Work Group to share restoration-related issues and benefit from the collective project development and implementation process. Listed in Table A.3 below is a summary of the group's organizational features related to restoration and their respective capacity to implement various phases of a restoration project. These organizations are listed here as potential Project Sponsors that will have the opportunity to use the approach as a resource for restoration project planning, implementation, and related monitoring. 
Table A.3. Other Restoration-Related Organizations in the Columbia River Estuary

\begin{tabular}{|c|c|c|c|c|}
\hline $\begin{array}{l}\text { Organization/ } \\
\text { Program }\end{array}$ & Features & Geographic Scope & $\begin{array}{l}\text { Targeted } \\
\text { Species }\end{array}$ & Relationship to Restoration Program \\
\hline Columbia Land Trust & $\begin{array}{l}\text { Private, non-profit conservancy organization active } \\
\text { in habitat protection and restoration in the Columbia } \\
\text { River estuary. The mission of the Land Trust is to } \\
\text { conserve important habitat areas together with local } \\
\text { communities by employing the tools of private } \\
\text { voluntary land conservation. }\end{array}$ & $\begin{array}{l}\text { Columbia River } \\
\text { Estuary }\end{array}$ & $\begin{array}{l}\text { No targeted } \\
\text { species }\end{array}$ & $\begin{array}{l}\text { Resource for land acquisition opportunities } \\
\text { and restoration planning in the Columbia } \\
\text { River estuary. Possess ability to present to } \\
\text { willing landowner a suite of conservation- } \\
\text { based alternatives through financial } \\
\text { incentives. }\end{array}$ \\
\hline $\begin{array}{l}\text { Columbia River } \\
\text { Estuary Study } \\
\text { Taskforce }\end{array}$ & $\begin{array}{l}\text { Local Council of Governments providing planning } \\
\text { assistance for the local jurisdictions of the Columbia } \\
\text { Estuary }\end{array}$ & $\begin{array}{l}\text { River Mile } 0 \text { to } 46 \\
\text { representing the } \\
\text { interests of both } \\
\text { Washington and } \\
\text { Oregon }\end{array}$ & $\begin{array}{l}\text { All species } \\
\text { dependent on } \\
\text { the resources } \\
\text { of the } \\
\text { Columbia } \\
\text { Estuary }\end{array}$ & $\begin{array}{l}\text { Resource for providing restoration site, } \\
\text { planning, project prioritization, and } \\
\text { monitoring of tidal wetlands. }\end{array}$ \\
\hline Ducks Unlimited & $\begin{array}{l}\text { A national organization dedicated to the } \\
\text { conservation of wetlands, Ducks Unlimited is } \\
\text { conducting a variety of restoration planning and } \\
\text { design work in the study area. They offer a variety } \\
\text { of technical services to landowners and } \\
\text { organizations conducting restoration work in the } \\
\text { study area. }\end{array}$ & National & $\begin{array}{l}\text { Waterfowl } \\
\text { and other } \\
\text { wetland } \\
\text { dependent } \\
\text { species }\end{array}$ & $\begin{array}{l}\text { Resource for providing technical assistance } \\
\text { for assessing restoration site potential, } \\
\text { planning, project prioritization, and } \\
\text { monitoring of tidal wetlands. }\end{array}$ \\
\hline Friends of Fox Creek & $\begin{array}{l}\text { Friends of Fox Creek is a community action group } \\
\text { that was organized in November 1991. Its mission is } \\
\text { to restore Fox Creek in Rainier for education, } \\
\text { wildlife habitat, community recreation and flood } \\
\text { protection. }\end{array}$ & Watershed & $\begin{array}{l}\text { No targeted } \\
\text { species } \\
\text { specifically }\end{array}$ & $\begin{array}{l}\text { Locally based group that has experience in } \\
\text { coordinating larger-scale restoration with } \\
\text { multiple groups. They have recruited the } \\
\text { resources of the COE Section } 1135 \\
\text { program to implement a restoration project } \\
\text { in Fox Creek. They have identified similar } \\
\text { restoration projects in the Rainier area that } \\
\text { could benefit anadromous fish and other } \\
\text { native species. }\end{array}$ \\
\hline
\end{tabular}




\begin{tabular}{ll}
\hline $\begin{array}{l}\text { Organization/ } \\
\text { Program }\end{array}$ & Features \\
\hline $\begin{array}{l}\text { Lower Columbia } \\
\text { River Watershed } \\
\text { Council }\end{array}$ & $\begin{array}{l}\text { Watershed Council representing the multiple } \\
\text { interests of Oregon side of the Lower Columbia } \\
\text { River }\end{array}$ \\
& \\
Nature Conservancy & $\begin{array}{l}\text { National organization that owns and manages } \\
\text { important tidal swamp habitat in the Columbia } \\
\text { Estuary; a valuable resource for understanding } \\
\text { processes and functions of remaining historical } \\
\text { features of lost habitat types. }\end{array}$
\end{tabular}

Natural Resource Primary resource locally for government-sponsored Conservation Service programs to conserve land, water, forests, wildlife (NRCS) and Soil and related natural resources.

Districts (SWCD)
North Coast Land

Conservancy
A long standing locally based conservation organization that owns parcels of land, primarily on the Oregon Coast.
Geographic Scope Targeted Species

Clatskanie Flats, Anadromous

Fox Creek,

Westport Slough, and Beaver Creek

National

County (SWCD) and Regional (NRCS)

No targeted species specifically

Species that contribute to the natural bio-diversity of a given region.

\section{Relationship to Restoration Program}

Have experience working with existing restoration authorities including the Army COE Section 1135 program. The Watershed Council worked with ACOE to reconnect a portion of the Westport Slough channel back to the tidal benefiting water quality and fish passage in the area.

Currently working on a bio-regional approach to prioritizing important ecological areas to focus their efforts.

Information that is produced from this effort should be linked to any prioritization process for future restoration projects. TNC can provide financial assistance for land acquisition of important habitat types in the study area. In addition they can provide information that characterizes the conditions of intact habitat "patches" that is a useful tool for restoration of areas adjacent to their property.

Local and regional resource for conservation incentive programs promoting the enhancement of habitat features relevant to salmon that include but not limited to: riparian plantings/fencing, erosion control, and conservation easement programs such as the Wetland Reserve Program

North Coast of

Oregon and

Anadromous

Fish and tidelands of the Native Plant Oregon side of the Species Columbia Estuary ecological features of Coastal and

Columbia Estuary habitats. Demonstrates capacity to administer purchases for environmental protection and restoration.
Possess knowledge of unique, local 


\begin{tabular}{|c|c|c|c|c|}
\hline $\begin{array}{l}\text { Organization/ } \\
\text { Program }\end{array}$ & Features & Geographic Scope & $\begin{array}{l}\text { Targeted } \\
\text { Species }\end{array}$ & Relationship to Restoration Program \\
\hline $\begin{array}{l}\text { North Coast } \\
\text { Watershed } \\
\text { Association }\end{array}$ & $\begin{array}{l}\text { Grass roots-based organization that identifies, } \\
\text { coordinates, and implements restoration projects } \\
\text { with multiple interests of the watershed. }\end{array}$ & $\begin{array}{l}\text { Watersheds of the } \\
\text { Oregon side of the } \\
\text { Columbia Estuary }\end{array}$ & $\begin{array}{l}\text { Anadromous } \\
\text { Fish }\end{array}$ & $\begin{array}{l}\text { Key resource for assessing the feasibility of } \\
\text { projects in the context of a given } \\
\text { communities needs. }\end{array}$ \\
\hline Pacific Joint Venture & $\begin{array}{l}\text { The Joint Venture is a coalition of private } \\
\text { conservation, waterfowl, fisheries, and agriculture } \\
\text { organizations working with government agencies to } \\
\text { protect and restore important wetland habitats. The } \\
\text { Joint Venture helps put together partnerships for a } \\
\text { variety of wetland-related projects, including habitat } \\
\text { restoration and enhancement on private and public } \\
\text { lands, acquisition of key areas for permanent } \\
\text { protection, and development of educational and } \\
\text { interpretive programs. }\end{array}$ & Regional & $\begin{array}{l}\text { Waterfowl } \\
\text { and other } \\
\text { wetland } \\
\text { dependent } \\
\text { species }\end{array}$ & $\begin{array}{l}\text { Potential cost share partner with available } \\
\text { funds and authorities to support restoration } \\
\text { project proposals. }\end{array}$ \\
\hline $\begin{array}{l}\text { Scappoose Bay } \\
\text { Watershed Council }\end{array}$ & $\begin{array}{l}\text { Representing the multiple interests of Scappoose } \\
\text { Bay, the watershed council facilitates the } \\
\text { development of important assessment products and } \\
\text { restoration projects. }\end{array}$ & Scappoose Bay & $\begin{array}{l}\text { Anadromous } \\
\text { Fish }\end{array}$ & $\begin{array}{l}\text { Local contact for scoping the feasibility of } \\
\text { restoration activities in the Scappoose Bay } \\
\text { system. }\end{array}$ \\
\hline Sea Resources & $\begin{array}{l}\text { Sea Resources is a nonprofit, community-based } \\
\text { education and watershed restoration organization } \\
\text { situated on the Chinook River (Baker Bay) in } \\
\text { Southwestern Washington. Currently, Sea } \\
\text { Resources' program emphasizes watershed } \\
\text { restoration and demonstrates how a community } \\
\text { project can teach a variety of disciplines and skills } \\
\text { necessary for salmon recovery. }\end{array}$ & Watershed-Based & $\begin{array}{l}\text { Native species } \\
\text { of the } \\
\text { Columbia } \\
\text { Estuary }\end{array}$ & $\begin{array}{l}\text { Resource for restoration planning in the } \\
\text { Estuary and associated effectiveness } \\
\text { monitoring. }\end{array}$ \\
\hline $\begin{array}{l}\text { The Wetlands } \\
\text { Conservancy }\end{array}$ & $\begin{array}{l}\text { The Wetlands Conservancy (TWC) is a non-profit } \\
\text { land trust. TWC owns and manages many wetlands } \\
\text { preserves in the Portland metropolitan area and } \\
\text { along the Oregon coast. In 2001, TWC launched the } \\
\text { "Oregon's Greatest Wetlands Project" and set an } \\
\text { ambitious goal of working with others to } \\
\text { permanently conserve } 12,000 \text { acres of wetlands in }\end{array}$ & State of Oregon & $\begin{array}{l}\text { Wetland } \\
\text { associated } \\
\text { species }\end{array}$ & $\begin{array}{l}\text { Resource for identification of sites, } \\
\text { ecosystem monitoring expertise, } \\
\text { community facilitation and liaison, project } \\
\text { planning, restoration and conservation } \\
\text { designs, specs and implementation, and } \\
\text { long- term management, maintenance, and } \\
\text { stewardship technical assistance. }\end{array}$ \\
\hline
\end{tabular}




\begin{tabular}{|c|c|c|c|c|}
\hline $\begin{array}{l}\text { Organization/ } \\
\text { Program }\end{array}$ & Features & Geographic Scope & $\begin{array}{c}\text { Targeted } \\
\text { Species }\end{array}$ & Relationship to Restoration Program \\
\hline & $\begin{array}{l}\text { Oregon. The "Oregon's Greatest Wetlands" Project } \\
\text { is an effort to identify, map, and gather information } \\
\text { on the state's most valuable wetlands. In order to } \\
\text { conserve and restore Oregon's wetlands, TWC is } \\
\text { working closely with local communities, land trusts, } \\
\text { watershed councils, individual landowners and state } \\
\text { resource managers to build local stewardship, } \\
\text { acquire and restore wetlands. }\end{array}$ & & & \\
\hline
\end{tabular}




\section{Appendix B}

Conceptual Ecosystem Model for the CRE 


\section{Appendix B: Conceptual Ecosystem Model for the CRE}

Section 5 of the Biological Assessment for the Columbia River Channel Improvements Project (COE 2001) describes the relationships among ecosystem components and the factors that determine salmonid production and ocean entry. A conceptual model was constructed of the Columbia River estuary ecosystem relationships that are significant for juvenile salmonids. The model provides a framework for evaluating potential environmental effects on listed salmonid species. In discussions of the complex nature of the lower Columbia River and its estuary, the science panel convened by SEI identified the need for a consistent framework for understanding the lower Columbia River ecosystem. The conceptual model discussed in this chapter is based on the agencies' discussions of a common framework. The framework is to be used to understand and explain the estuarine ecosystem and its functions as they relate to salmonids.

Figure B.1 depicts flows from the general processes (ocean and river) through the more specific characteristics of this ecosystem. It is also compatible with other conceptual tools that may be used in biological assessments, including the NMFS' concept of Properly Functioning Conditions (PFC). The PFC format for large river estuarine ecosystems is discussed further in Section B.2.

The conceptual model for juvenile salmonids of the lower Columbia River provides an integrated diagram of the major ecosystem links that affect ecosystem structure and function as they relate to juvenile salmonid production and ocean entry. The specific objectives of the lower Columbia River model are to:

- Provide an ecosystem-level scientific framework for evaluating the Project

- Identify links among physical-chemical and biological indicators

- Aid in the identification of ecosystem-based processes that link salmon and potential effects of the Project

- Develop a systematic methodology to evaluate monitoring and adaptive management opportunities

The conceptual model is generally formatted to describe the present state of the ecosystem, using general factors and identifying how they influence a specific function, as shown below:

\section{Controlling Factors $\rightarrow$ Ecosystem Structure $\rightarrow$ Ecosystem Function}

The controlling factors refer to those general physical processes that influence all river conditions. The ecosystem structure refers to how those factors are manifest, specifically in the 
lower Columbia River system. The ecosystem function is then determined by output of indicators specific to the ways in which the ecosystem structure functions to produce salmonids for ocean entry.

The goal of the model is to present a clear, scientifically based hypothesis in diagram form that illustrates major connections among processes, indicators, and pathways within the system. Because of the complexity of the ecosystem, these connections are illustrated in a series of figures representing a set of linked submodels based on the functional pathways of the system. These pathways include processes within the river system (e.g., habitat formation, tides, bedload transport, accretion/erosion); specific components, or indicators, within the system (e.g., habitat types, food types, physical properties); and the pathways through which these processes and indicators combine to affect the ecosystem (e.g., primary productivity, food web). The processes and indicators used in the conceptual model are introduced in bold type throughout this chapter. Habitat types are shown in italics on first reference.

Figure B.1 illustrates the relationships among the major functional pathways that affect salmonids in the lower Columbia River. These pathways support the growth and survival of juvenile salmonids, which then result in the output shown in Figure B.1 and in the model - i.e., juvenile salmonid production and ocean entry. Salmonid production and ocean entry depend on several functions, including development of habitats, production of food to fuel the food web, and ability of salmonids to access and use these habitats. The culmination of these functions results in growth and survival of salmonids and their ultimate entry into the ocean.

Figures B.2 and B.3 illustrate the habitat-forming processes and the various indicators that lead to development of the habitat types that support juvenile salmonid growth and survival, again leading to the output of juvenile salmonid production and ocean entry. These figures depict the ecosystem function as it relates to salmonids in the action area. Figure B.2 presents these processes for the ocean areas, while Figure B.3 presents them for the areas that lie within the Columbia River system. Other figures in this section illustrate each of the major function pathways.

The requisites provided to salmonids in the lower Columbia River ecosystem are a function of the ability of salmonids to access habitats (i.e., habitat opportunity) and the amount of food available within these habitats (i.e., habitat capacity) (Bottom et al. 2001). In turn, opportunity and capacity depend on the development and functioning of viable habitats. These habitats are formed and maintained by physical and chemical forcing factors. Significant interactions affect both the development of habitat and the support provided by habitats to salmonids. These interactions include habitat succession rates and patterns, disturbance regimes, landscape connectivity, and diversity among salmonid ESUs and DPSs. The model highlights the complexity of the factors supporting juvenile salmonid production and ocean entry. 

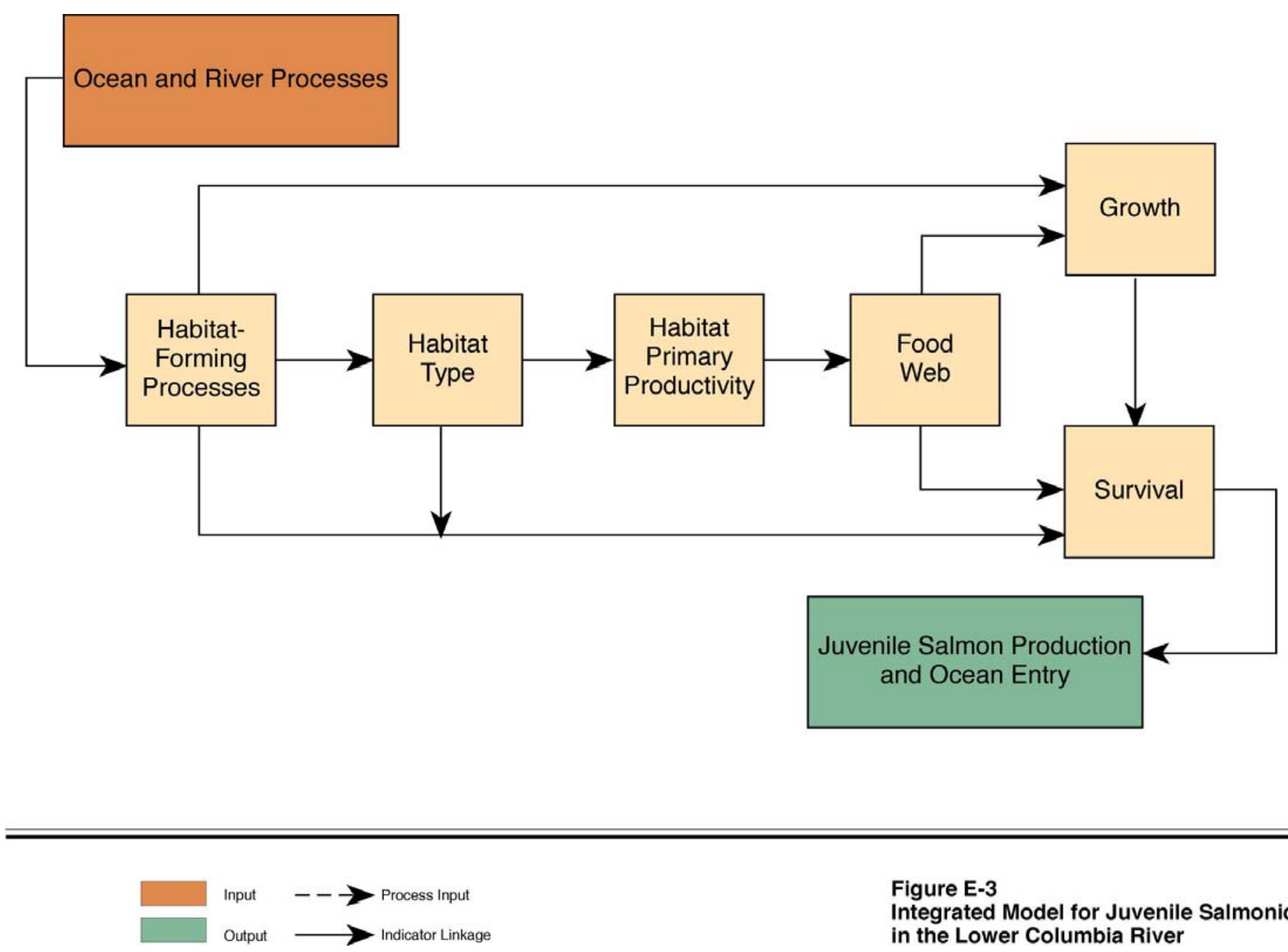

\section{Figure E-3}

integrated Model for Juvenile Salmonids

in the Lower Columbia River

Figure B.1. Integrated Model for Juvenile Salmonids in the CRE. 


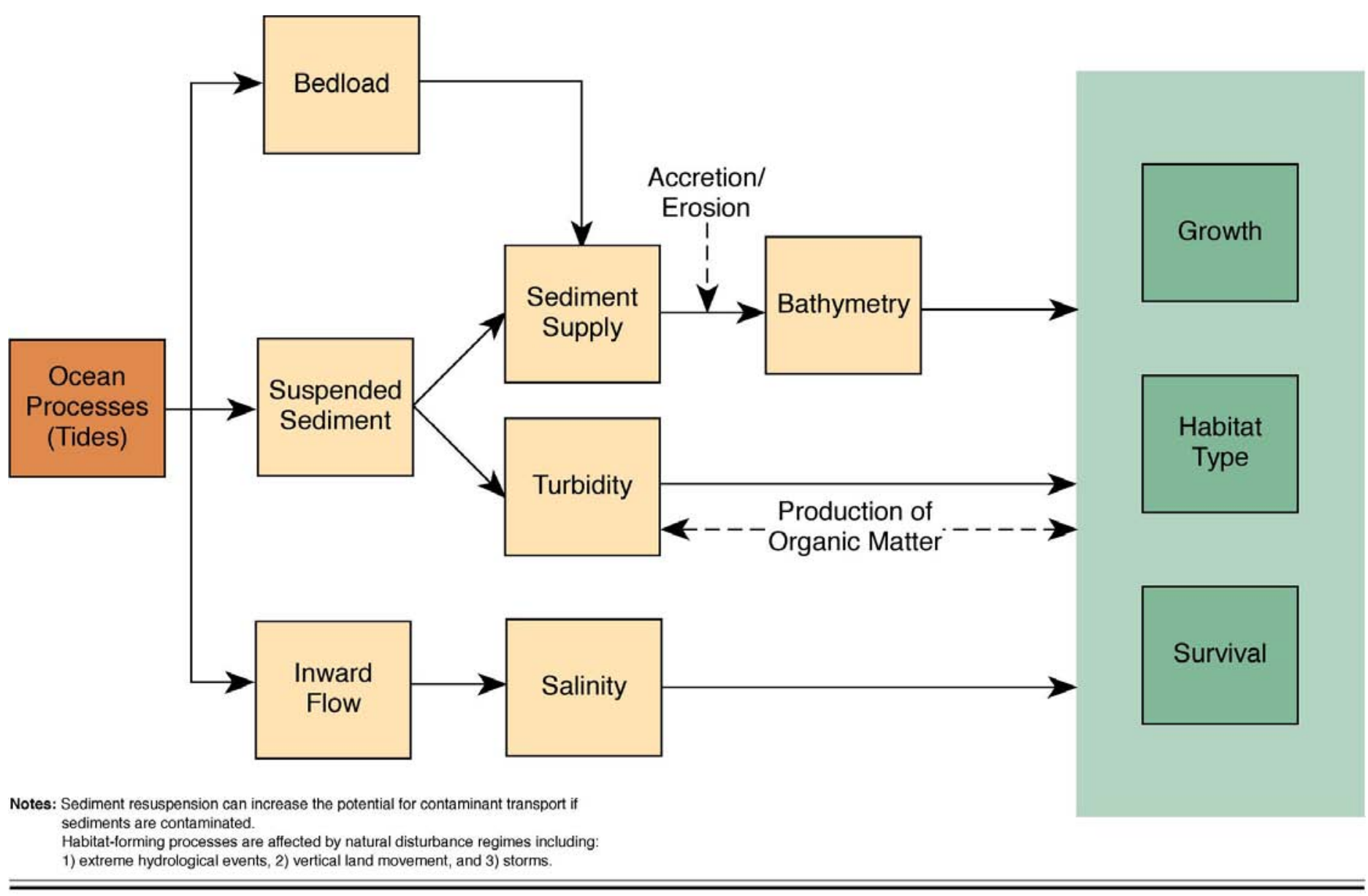

$$
\begin{aligned}
& \text { Sediments are contaminated. } \\
& \text { Habitat-forming processes are affected by natural disturbance regimes including: }
\end{aligned}
$$

1) extreme hydrological events, 2) vertical land movement, and 3) storms.

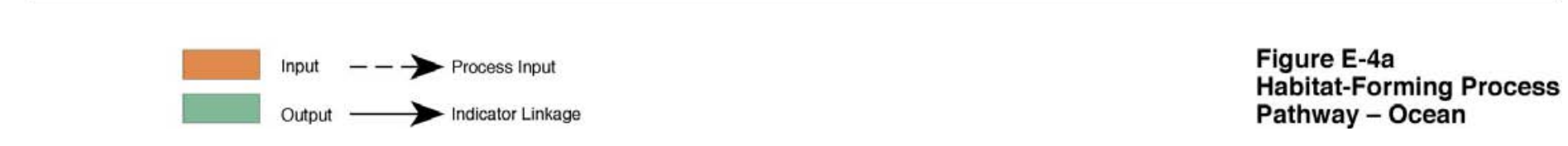

Figure B.2. Habitat Forming Process Pathway - Ocean. 


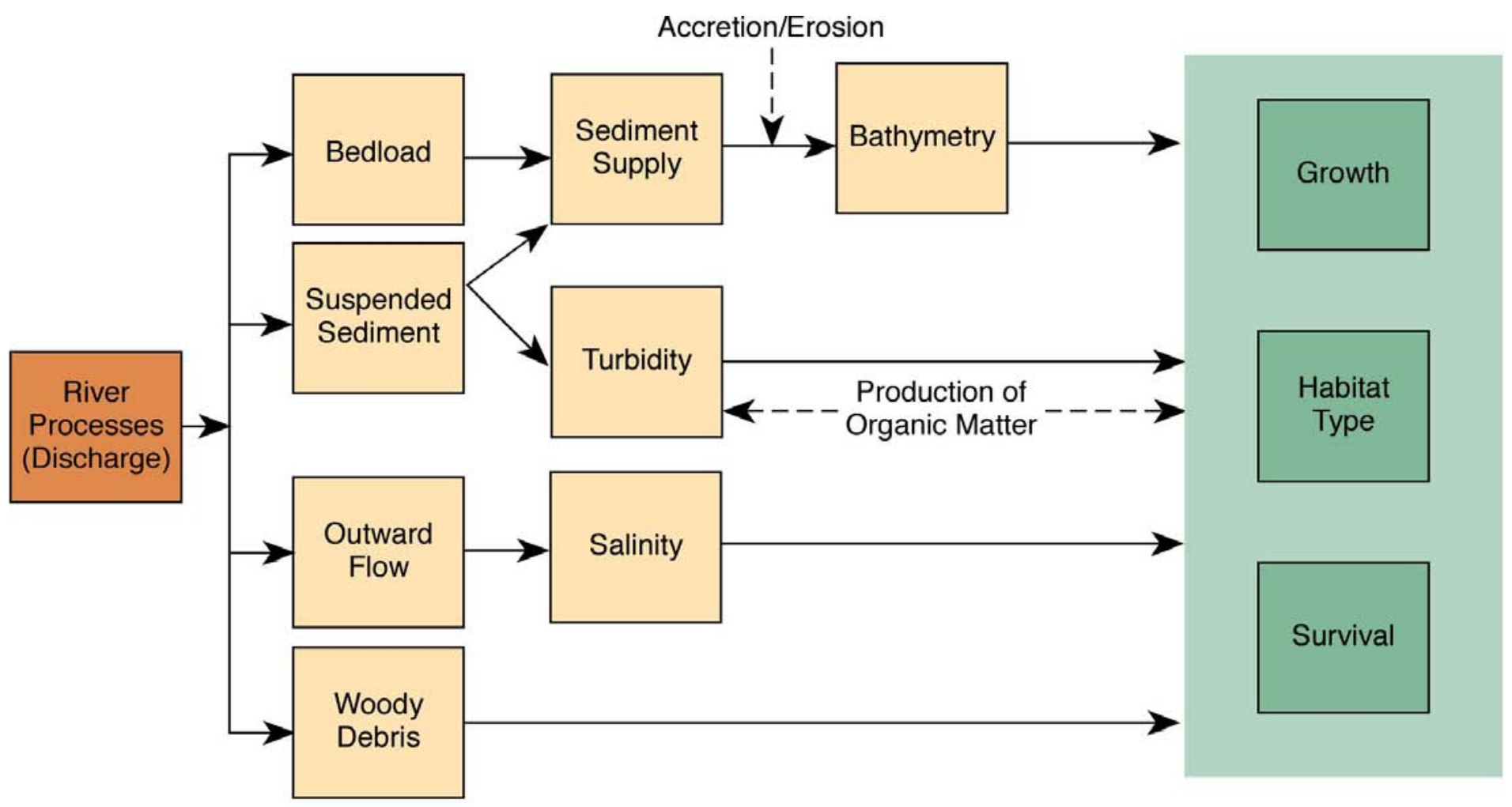

Notes: Sediment resuspension can increase the potential for contaminant transport if

sediments are contaminated.

are affected by natural disturbance regimes including:

1) extreme hydrological events, 2) vertical land movement, and 3) storms.

$\square$ Input $\quad-\rightarrow$ Process Input

Output $\longrightarrow$ Indicator Linkage
Figure E-4b

Habitat-Forming Process

Pathway - River

Figure B.3. Habitat Forming Process Pathway - River. 


\section{B.1 Habitat-Forming Processes}

Habitats are formed primarily by the interaction of hydrodynamic forces and sediment supply. In the lower Columbia River, both the river and the ocean influence the estuarine hydrodynamics. River discharges and volumes are regulated by precipitation, temperature (i.e., freeze and thaw), and reservoir operations. Ocean processes, including tidal action and waves, interact with river processes, including currents and sediment transport, in the lower Columbia to produce the estuary's complex hydrodynamics. The net result is deposition (accretion) of suspended sediments to form flats and carving (erosion) to form shallow and deep channels. Where sediment deposits can form islands, marsh and swamp vegetation can develop. These marshes and swamps are dissected by shallow channels, which allow fish access to edges of the vegetated areas.

The indicators and processes involved in the formation and maintenance of lower Columbia River habitats are illustrated in the Habitat-Forming Processes Pathway shown in Figures B.2 and B.3. The main factors affecting or explaining habitat development include salinity and bathymetry (i.e., elevation of substrate). Salinity and bathymetry are indicators of system function. Additional indicators include suspended sediment, bedload, woody debris, turbidity, and accretion/erosion. Woody debris is a special case of a habitat-forming indicator that is directly input into the estuary from upstream sources.

Shallow water and flats form in intertidal sandy or muddy areas where sediments are somewhat unstable and where the elevation is not high enough for emergent marshes to develop. If the turbidity levels are low enough to allow sufficient light penetration for plant growth, these areas may develop submerged vegetation such as eelgrass.

Bedload transport describes the process through which the channel bottom sands are moved along the surface of the riverbed. In sandy riverbeds, like those in the lower Columbia River, bedload transport shapes portions of the bed into a series of sand waves. The hydraulic forces of the river move these waves downstream as sediment erodes from the upstream face, deposits in the downstream trough, and is then buried by additional material eroded from the upstream face. The topography created by these sand waves is the bathymetry of the river.

The movement and deposition of large woody debris are also affected by the hydrologic process. It is deposited on the flats, along channel edges, and in marshes and swamps. Woody debris creates a vertical structure to which fish often orient and also provides "micro" habitats that can trap organic matter, which can be rich in invertebrates.

Another important factor in habitat development is the mixing of freshwater and saltwater in the lower river, which results in a salinity gradient in the estuary. The zone of mixing varies significantly in location, depending on river flow and tides. Because it is denser than freshwater, saltwater moves upstream along the bottom where it forms a salt "wedge" below the overlying layer of freshwater. Intense mixing proportional to river depth occurs at the area between freshwater and saltwater. Because plants and animals are adapted to certain salinity ranges, the salinity level, as well as seasonal and spatial patterns, strongly influences where species occur in the lower Columbia River. 
As in many other estuaries, turbidity from suspended sediment and plankton is moderate to high in the lower Columbia River. High river flows and heavy wind and wave activity can increase turbidity significantly. Because plants require light to grow, turbidity affects how deep plants can grow below the water surface. Higher turbidity means that plants can grow only very near the surface of the water. Rooted aquatic plants, such as eelgrass (Zostera marina), are generally limited to very shallow depths in the estuary because of turbid water (Dennison et al. 1993).

Table B.1 is a list of salinity ranges that occur in estuaries. Of relevance to juvenile salmonids is the oligohaline zone, brackish water areas of only slight salinity, where juvenile salmonids go through a physiological transition to a saltwater environment. Juvenile ocean-type salmon may spend a considerable period of time in the oligohaline zone, where they require adequate food supplies and refuge from predators to survive and grow.

Table B.1. Salinity Zones (modified from Cowardin et al. 1979)

\begin{tabular}{ll}
\hline Zones & Salinity Range $(\mathrm{ppt})$ \\
\hline Hyperhaline & $>40$ \\
Euhaline & $30.0-40$ \\
Mixohaline (brackish) & $0.5-30$ \\
Polyhaline & $18.0-30$ \\
Mesohaline & $5.0-18$ \\
Oligohaline & $0.5-5$ \\
Fresh water & $<0.5$ \\
\hline
\end{tabular}

In the Habitat-Forming Processes Pathway (see Figures B.2 and B.3), all of these dynamics and interactions culminate in the expression of habitat types important to salmon in the lower Columbia River. The habitats created are shown in the Habitat Type Pathway (Figure B.4) and relative to elevation ranges (Figure B.5). 


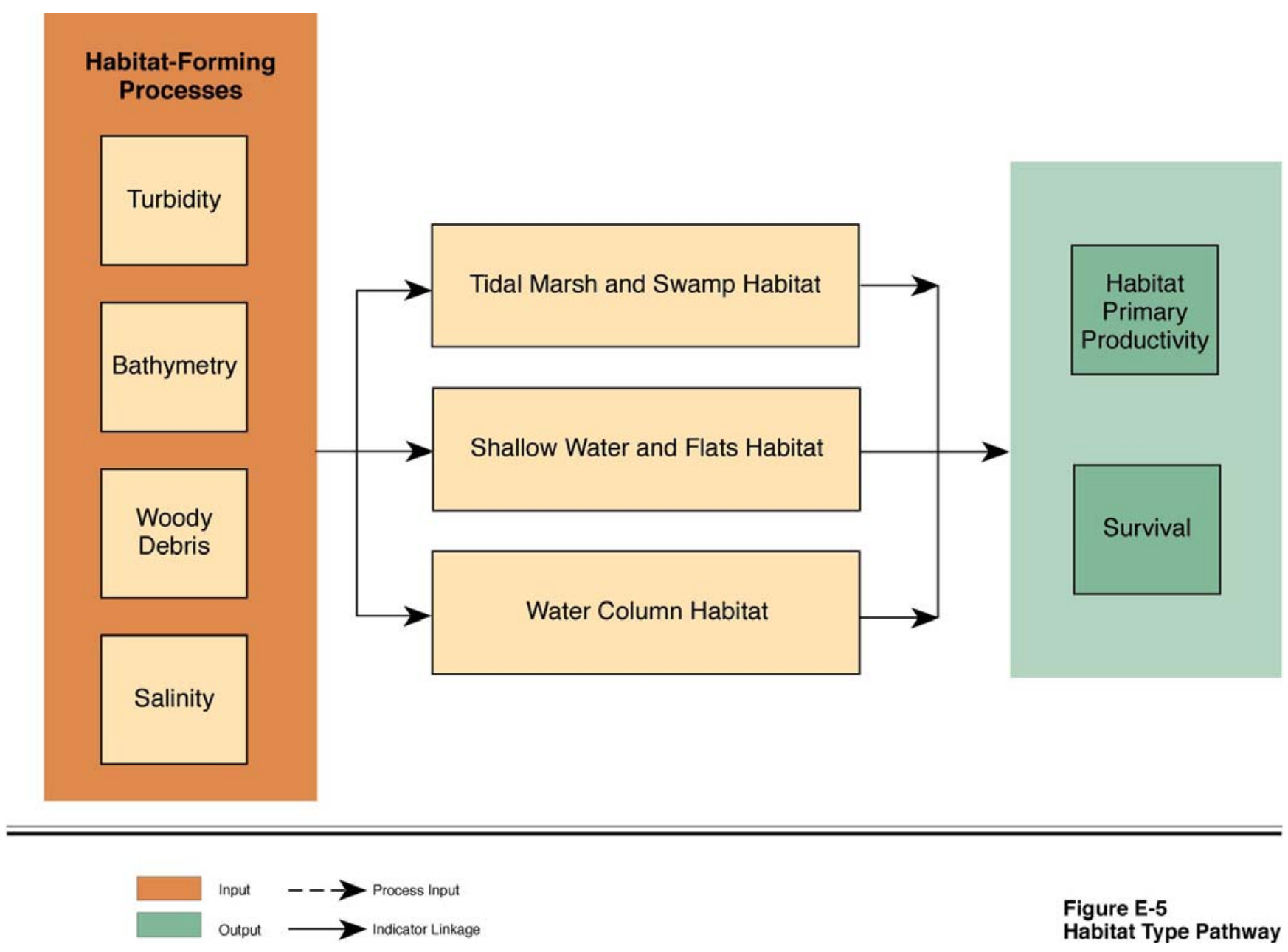

Figure B 4. Habitat Type Pathway. 


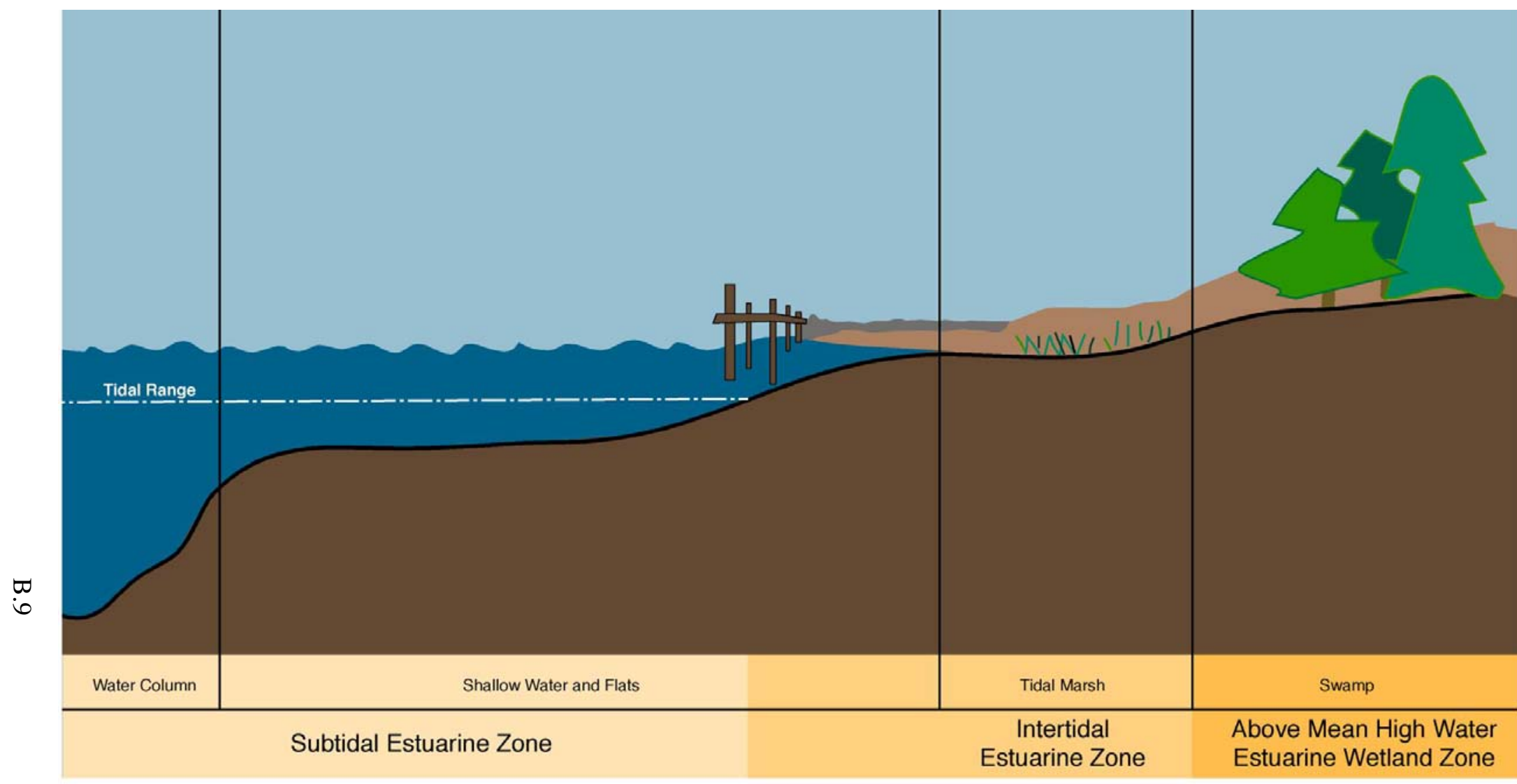

Figure E-7

Major Habitat Types in the System

Figure B 5. Major Habitat Types. 

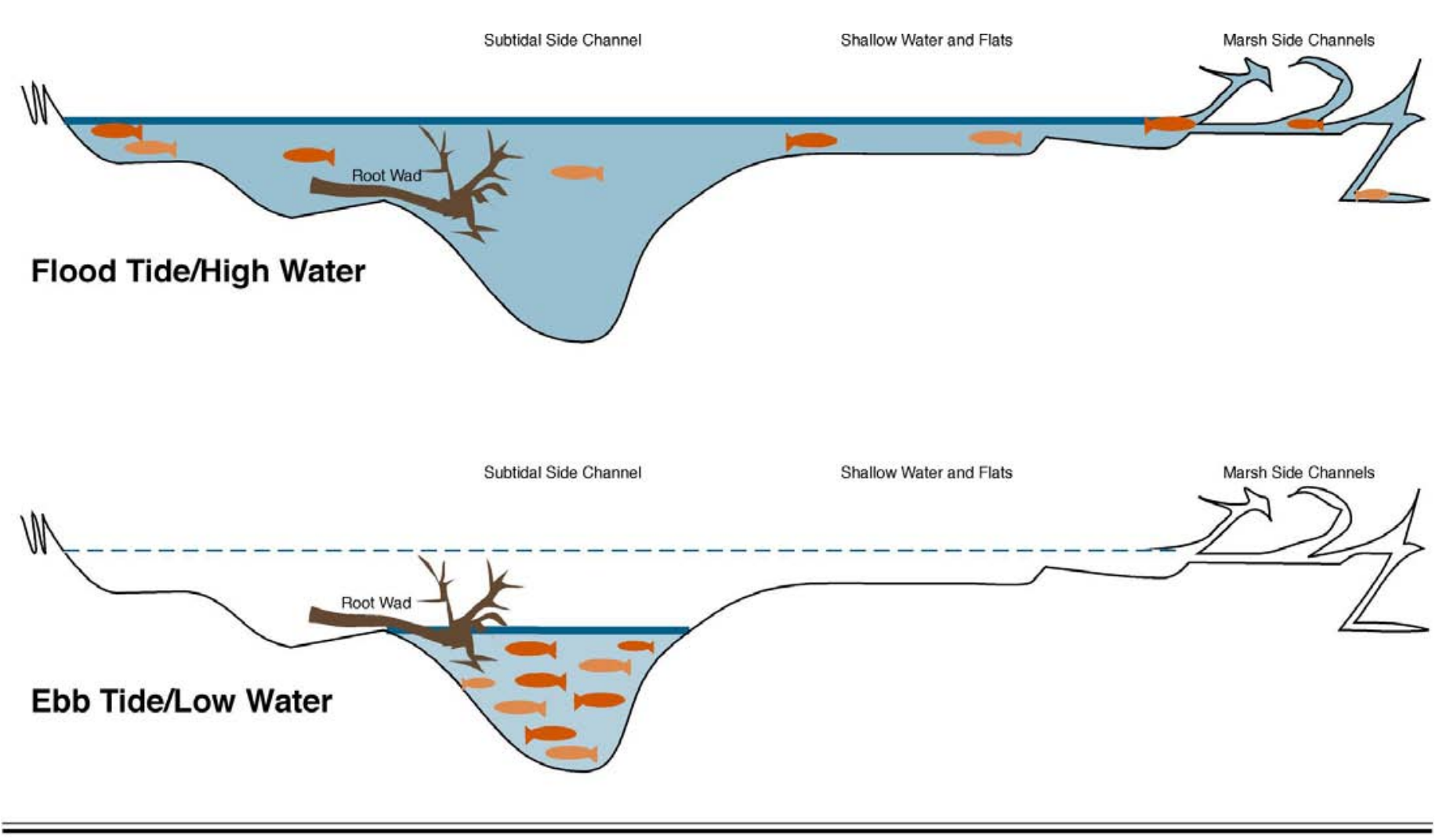

\section{Figure E-10}

General Pattern of Lower Columbia River Use by Juvenile Salmonids

Figure B 6. General Pattern of Use. 


\section{B.2 Habitat Types}

The habitats most directly linked to salmonid in the lower Columbia River include the tidal marshes and swamps, shallow water and flats, and the water column. As described in Section B.1, these habitats are the result of highly dynamic physical processes interacting in the river and ocean of the action area. Habitat types are generally defined by specific elevation ranges (Figure B.5 and Figure B.6).

Tidal marshes and swamps generally occur from about MHHW. Tidal marshes begin at lower tidal elevations, slightly above MLLW although rare at lower than these elevations, and swamps occur at or above MHHW (Thomas 1983). Thomas (1983) based these characteristics on a comparison of 19 vegetation types where low, medium and high elevations are based on a diurnal range (MLLW-MHHW) averaging 8 feet (where low equals 2.5 to 4 feet above MLLW; medium equals 4 to 6.5 feet above MLLW; and high equals above 6.5 feet). Ocean-type juvenile salmonids use the edges of these marshes to feed, and the edges of shallow channels within the marshes as refuge and feeding areas. Consequently, access to the edges at high tide and development of low-tide refuge areas near or within marshes are critical to lower river ocean-type juveniles. Channel order (the number and width of channels) and channel depth are also functional characteristics of a marsh area. The aquatic edge is considered to be an important factor governing the exchange of organisms, and the connectivity associated with the channels offers more opportunity to marsh access (Shafer and Yozzo 1998). Although there are no empirical data on this relationship for the lower Columbia River, smaller marshes would provide limited salmonid access and only limited nearby low-tide refuge areas. Large marshes provide access to a much greater amount of edge and provide low-tide refuge.

Tidal marshes can be divided into saltwater marshes and freshwater marshes, each characterized by a distinctive vegetation type. Tidal marshes include tidally influenced areas all the way up to Bonneville Dam, as well as extensive tidal freshwater marshes in the lower Columbia River, particularly those in Cathlamet Bay. Shallow water and flats occur throughout the intertidal zone and into the shallow subtidal zone in waters up to 6 feet deep. Benthic algae (largely benthic diatoms) develop on tidal flats and in the shallow subtidal zone within the system.

Water column habitat refers to waters that are greater than 6 feet deep and can be characterized by depth. For example, the upper 3 to 10 feet of the water column can have a very different community from that found at greater depths. The stratification is caused both by the salinity variation and the light penetration by depth. The water column habitat is essentially the location where phytoplankton and floatable organic matter occur within the lower Columbia River system. Both phytoplankton and zooplankton respond to salinity changes within the habitat. Freshwater plankton dominate the fresh and oligohaline portions of the water column upstream, and plankton tolerant of greater salinity dominate the estuary and the river mouth of water column habitats.

\section{B.3 Habitat Primary Productivity Pathway}

A major function of the habitats is to produce food used by the ecosystem. Habitat primary productivity refers to the amount of material (biomass) produced over time during plant growth that occurs within each habitat type. Primary productivity is driven by light (Figure B.7) and is supported by inorganic nutrients (e.g., nitrate, phosphate). Inorganic nutrients enter the system from the upstream 
watershed and the downstream ocean currents and through the breakdown and recycling of organic matter within the system. Factors that affect the distribution of the plants within the system include the habitatforming processes of sedimentation, erosion, salinity, and turbidity (see Figures B.2 and B.3). One example of the interaction of these processes is that, as turbidity is increased, light in the water column is reduced (Figure B.7). This can result in less plant growth as well as limit the depth at which plants will grow. The Habitat Primary Productivity Pathway is illustrated in Figure B.7.

Phytoplankton are the primary producers within water column habitat. Phytoplankton are singlecelled plants, primarily diatoms, that drift within the water column. There are two types of phytoplankton in the lower Columbia River: imported phytoplankton, which are freshwater species produced in large quantities in the upstream watershed (particularly in the reservoirs behind the mainstem dams), and resident phytoplankton, which are produced within the lower Columbia River. Resident species can be freshwater, euryhaline, or marine species.

Primary productivity within the shallow water and flats habitat results mostly from benthic algae, single-celled plants in or on the sediments. Shallow water habitats can also produce filamentous algae and flowering grasses such as eelgrass; however, the majority of primary productivity within the river's shallow water areas comes from benthic algae.

Primary productivity within tidal marsh and swamp habitat comes from the marsh and swamp vegetation, which includes emergent plants, shrubs, and trees.

As illustrated in the Food Web Pathway (Figure B8), live plant material and detritus are the primary sources of organic matter in the food web used by salmonids in the lower Columbia River.

\section{B.4 Food Web Pathway}

Another key function of the lower Columbia River is to provide for salmonids. A food web reflects who eats what in an ecosystem. It helps develop an understanding of the pathways by which trophic groups of the food web obtain food. In addition, when habitat types and habitat-forming processes change over time and affect productivity patterns, the resulting food web shifts can provide insight about processes that potentially limit the growth of groups within the food web. 


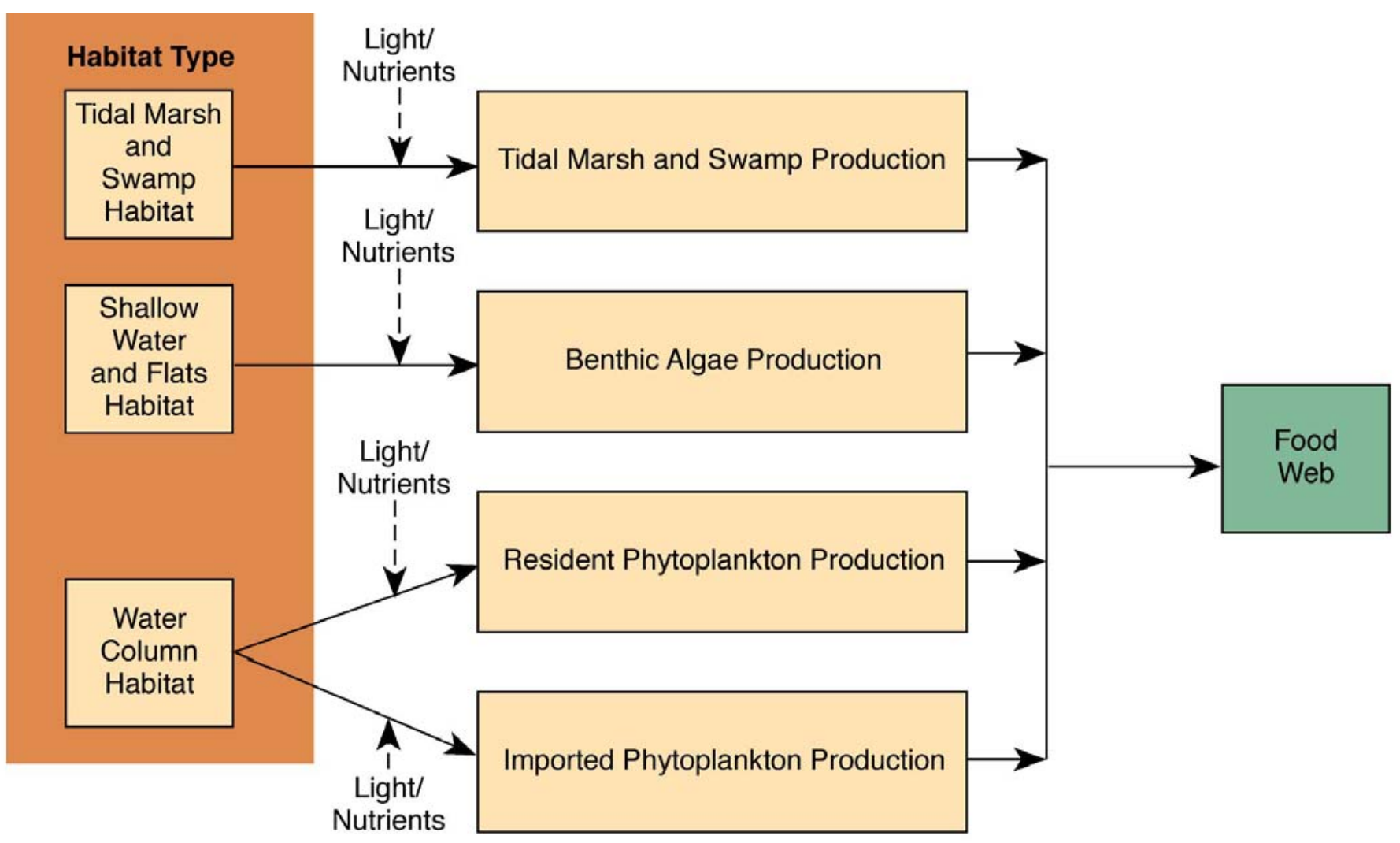

Figure E-6

Input $-\rightarrow$ Process Input

Habitat Primary Productivity Pathway

Figure B 7. Habitat Primary Productivity Pathway. 


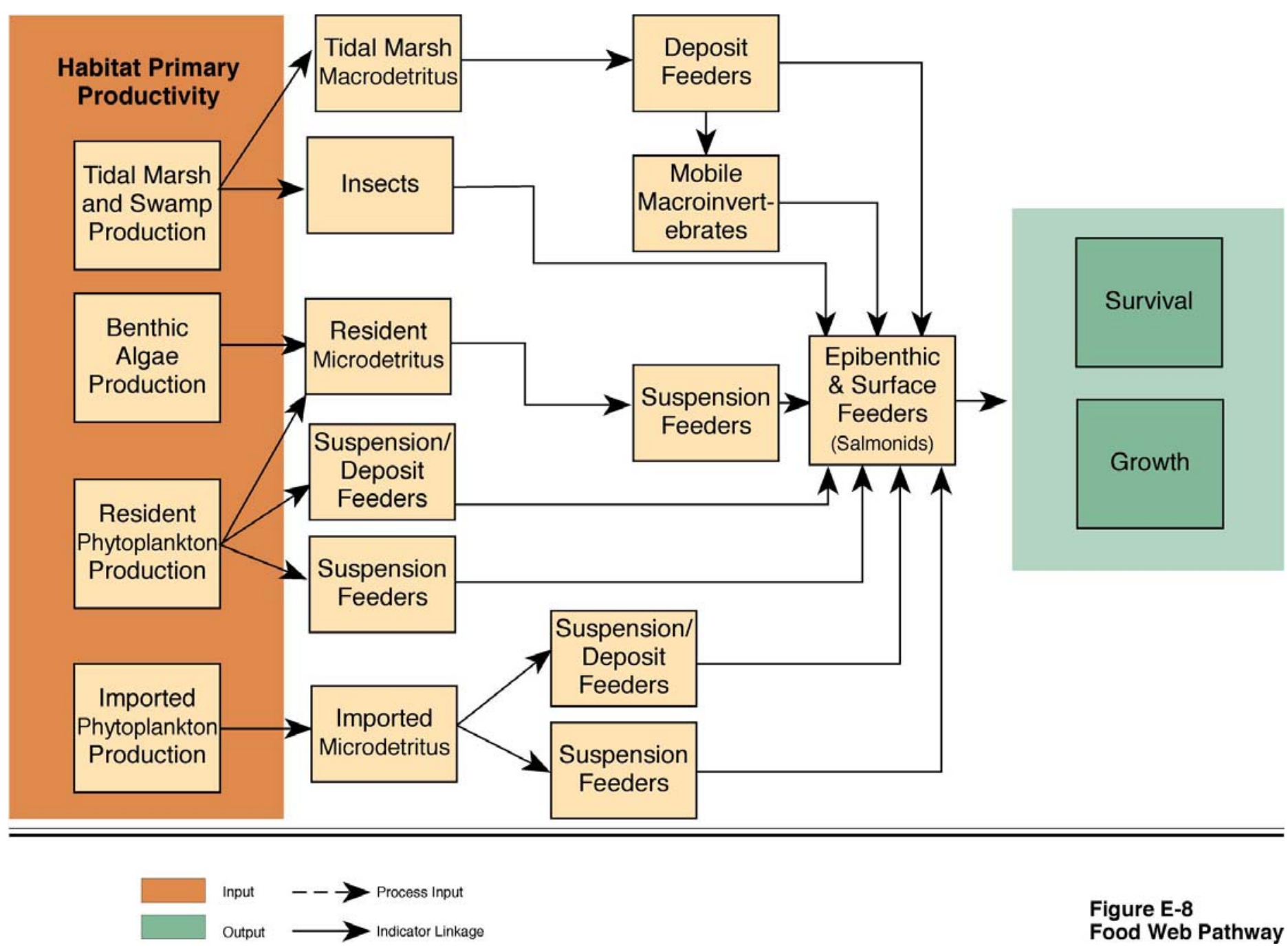

Figure B 8. Food Web Pathway. 
The base of any food web is the plant material produced over time or the primary productivity within each habitat type. This food web base also includes detritus (dead plant material). Macrodetritus in the system are large, complex forms of dead plants, primarily in tidal marsh macrodetritus. Microdetritus are dead, simple-celled plant materials or organic particles. Microdetritus can be in the form of imported microdetritus if they are derived from imported phytoplankton, or resident microdetritus if they are derived from resident phytoplankton. Small animals that shred the larger plant matter and microbes, including bacteria, protozoa, and fungi, facilitate the breakdown of detritus. In addition to making the organic matter useful to the food web, these breakdown processes recycle inorganic nutrients needed by the plants for primary production.

As illustrated in the Food Web Pathway (Figure B.8), juvenile salmonids are members of a complex food web in the lower Columbia River. The model represents only the salmonid portion of the total food web for the system, which is far more complex. The organic energy sources at the base of this web are shown on the left side of the figure and, as stated above, are from the primary producers of biomass as depicted in the Habitat Primary Productivity Pathway (Figure B7). The model illustrates energy transfers through live plants that can be eaten directly or detritus that can be incorporated into the food web through detritivores (animals that eat dead and decaying plants and animals).

Although the Food Web Pathway does not show the relative amounts of food energy derived from each primary producer type, it does illustrate that salmonids can and do eat invertebrate prey species that are supported by resident and imported plankton, detritus, and tidal marsh and swamp plant material. The relative amount of food and food energy depends on the abundance of each resident habitat type (e.g., tidal marshes) and the input of nonresident material from upstream sources. Input of nonresident material is determined from upstream production, primarily by production in the reservoirs behind the dams, which is regulated by Bonneville Dam flow rates.

Several types of feeders make up the next level up the food chain from the primary producers and their detritus. For purposes of the conceptual model, the next level has been grouped as follows:

Mobile macroinvertebrates are large epibenthic organisms that reside on the river bottom and feed on bottom sediments. The main examples of macroinvertebrates in the lower river include sand shrimp (Crangon franciscorum), mysids (Neomysis mercedis), and Dungeness crab (Cancer magister). Mysids are the primary macroinvertebrates that are relevant to the salmonid food web.

Deposit feeders are benthic animals that feed by consuming organic matter in sediments. For this conceptual model, the term deposit feeders refers to both surface and subsurface deposit feeders, which include marine annelids (polychaetes), and freshwater annelids (oligochaetes), and benthic crustaceans.

Suspension feeders are organisms that feed from the water column itself. For zooplankton and benthic/epibenthic organisms, this is accomplished primarily through "filter feeding" (extracting organic matter from the water column by pumping or siphoning the water through their systems). Among the most abundant species found in the stomachs of salmonids is the planktonic cladocera suspension feeder Daphnia pulex. 
Suspension/deposit feeders are benthic and epibenthic organisms that feed on or at the interface between the sediment and the water column. Perhaps the most abundant species found in the stomachs of salmonids is the benthic amphipod Corophium salmonis.

Floating insects (larvae and adults) appear to be important in the diet of most of the species and age classes in the salmonid food web. Many of these insect types feed on live tidal marsh plants.

As described in Section 2, subyearling Chinook, an example of juvenile salmonids in general, feed primarily on the bottom but in shallow water while they are in the lower Columbia River, whereas older (yearling) fish of all species feed primarily on zooplankton in the water column.

Where these prey species are found is also important. Because outmigrating juvenile salmon are often found in the upper 6 feet of the water column, they probably do not eat benthic (bottom dwelling) prey in deeper parts of the estuary (i.e., more than 6 feet deep). Consequently, the primary depth range for salmonids feeding on benthic prey is the intertidal zone and down to a depth of about $6 \mathrm{ft}$ below Extreme Lower Low Water. Insects, Corophium, and mysids located in shallow habitats such as tidal marshes, tidal channels, and flats are more available to salmonids at higher tides. On the other hand, planktonic prey such as Daphnia and copepods are available at any stage of the tide.

\section{B.5 Growth Pathway}

Salmonid feeding results in growth of the animals in preparation for their outmigration to the north Pacific. The Growth Pathway depicted in Figure B.9 incorporates feeding as well as other factors that are involved in producing salmonid growth in the lower Columbia River.

The inputs leading to the Growth Pathway (Figure B.9) indicate the progression from physical factors involved in defining habitats in the lower Columbia River through the way in which these habitats work to produce food for salmonids. The Growth Pathway highlights the factors involved in producing both the appropriate amount and type of food prey and the access by juvenile salmonids to productive feeding areas.

The characteristics of the food web, such as the abundance of insects versus the biomass of nonresident microdetritus and where prey and other nutrients are distributed, are important in determining the relative contribution of these food sources to the growth of salmonids. The "Food Abundance and Distribution" and "Habitat-Specific Food Availability" boxes in the Growth Pathway (Figure B.8) address these feeding factors. The actual location and structure of feeding habitats are important because salmonids need first to be able to access feeding habitat and, while there, be able to find the prey items.

Salmonids are adapted for using a complex mosaic of many habitat areas as they migrate downstream and during their residence in riverine and estuarine systems in the Pacific Northwest. Therefore, coupled with habitat-specific food availability, feeding habitat opportunity needs to exist for salmonids to feed within the set of habitats. As described in Section 2, juvenile salmonids primarily frequent very shallow water areas, especially the subyearling Chinook. They benefit most from prey produced in tidal marshes and marsh channels, on the edges of natural side channels, and on flats (Figure B.4). When water level is low, salmonids are believed to congregate at the edges of natural side channels and pools, which become low-tide refuges. 
This mosaic of habitats used by salmonids is referred to as habitat complexity. An absence or reduction in the natural complexity of habitats available may affect the salmonids' ability to reach food resources needed for growth. Conveyance is the opportunity for salmonids to move over flats and into tidal marsh systems as the water level rises and falls with the tide and with river flow (Figure B.5).

Connectivity refers to links and spatial arrangements among habitats in the mosaic of changing habitat areas. For juvenile salmonids in the lower Columbia River, this refers to favorable access among viable feeding, rearing, and refuge habitats along the migratory corridor. Blockages, interruptions of corridors, or modifications of habitat may prevent or limit access to productive feeding habitats. For example, a culvert may block fish access to tidal marsh behind a river levee. Large numbers of overwater structures may limit the ability to migrate or the migration habits of fish traveling along the shoreline. Because fish are adapted to use a wide but linked set of habitats, maintaining access among habitat types is important to feeding habitat opportunity. Connectivity is illustrated in the Growth Pathway (Figure B.9).

Low current, shallow areas provide productive feeding areas for salmonids. Available information suggests that velocities of 30 centimeters per second or less are best for optimal foraging opportunity (Bottom et al. 2001). Because salmonids are visual predators, turbidity and uneven bathymetry may limit their ability to prey. The concepts of velocity field, shallow bathymetry, and turbidity are illustrated in boxes at the left of the Growth Pathway (Figure B.9).

Finally, there are energy costs that each individual animal expends to feed. These include locating prey, feeding behavior, avoiding predators, and processing energy from prey consumed. In general, fish prefer high-energy food that provides the most energy per unit of effort. Anything less than this will, theoretically, produce suboptimal growth rates. 
$\varpi_{\infty}^{\infty}$

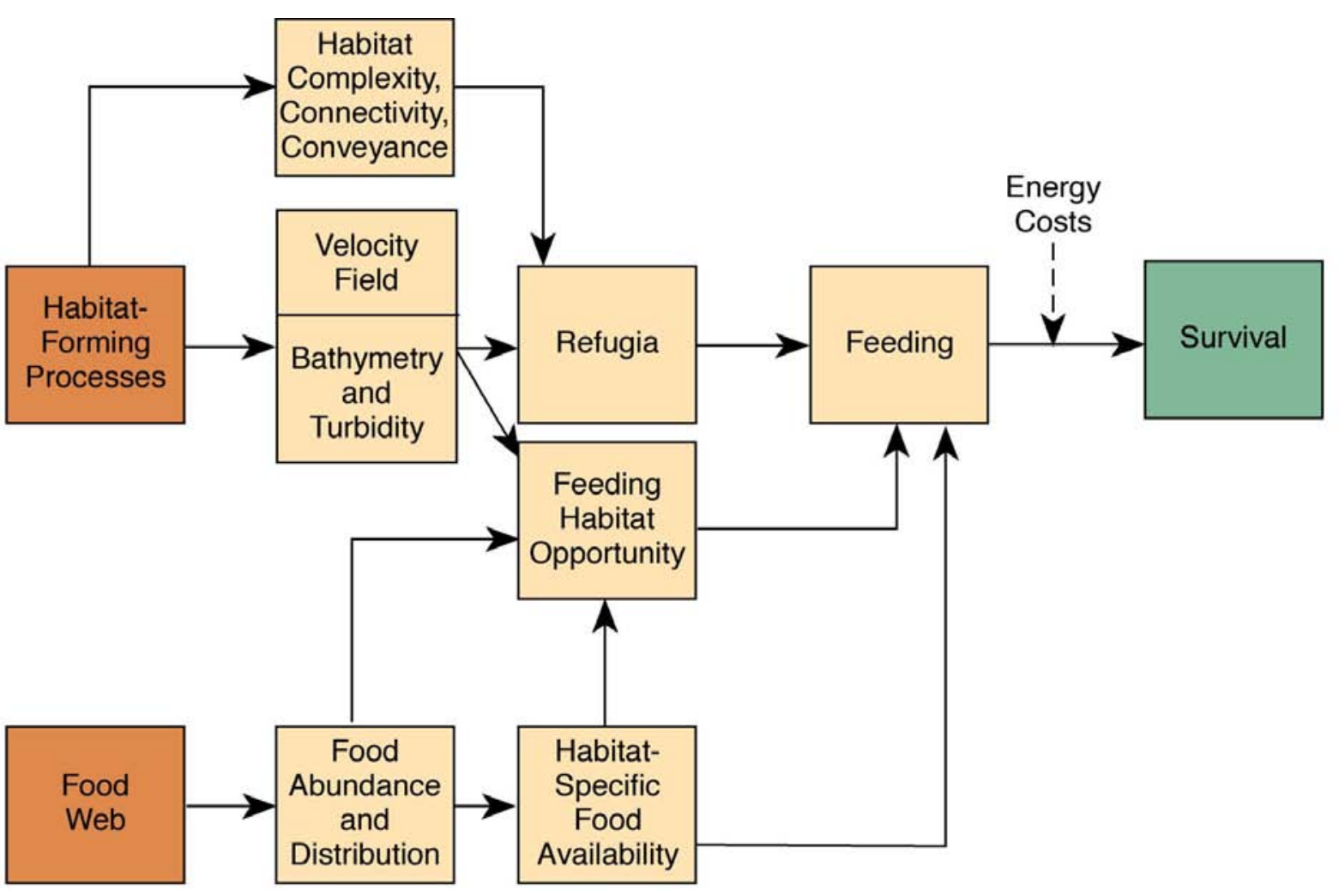

Input $--\rightarrow$ Process Input

Output $\longrightarrow$ Indicator Linkage
Figure E-9

Growth Pathway

9. Growth Pathway.

Figure B 


\section{B.6 Survival Pathway}

Besides growth, a variety of factors interact to affect the ultimate survival of salmonids in the lower Columbia River. The Survival Pathway (Figure B.10) shows the links among these factors.

Salmonid survival depends on the ability of fish to grow and migrate through the lower Columbia River system. As shown in the previous pathways, a complex set of factors can control or affect growth and migration.

Factors that can negatively affect survival include contaminants, predation, suspended solids, temperature and salinity extremes, stranding, entrainment, and competition. These factors are discussed below.

Contaminants include chemicals that can affect the health of salmonids. They can be taken up directly through the water column or through contaminated prey (food web). The prey of juvenile salmonids may obtain contaminants via their food. For example, contaminants deposited on the bottom along with organic matter may be ingested by deposit-feeding animals, which are in turn eaten by juvenile salmonids. These contaminants may affect the health (physiological integrity) of fish and may result in disease as well as a reduced ability to physiologically adapt to saltwater, avoid predators, forage effectively, and seek and find shelter.

Predation is a major factor affecting salmonid survival in the lower Columbia River. Birds, including Western grebes, cormorants, gulls, terns, and great blue herons, are known to prey on small fish that may include young salmon. Fish predators are less well known, but larger fish, including sculpins, have been documented as having salmon in their guts.

Suspended solids, which can be a major contributor to turbidity, affect migratory ability by reducing the ability of salmonids to see prey. Data indicate that the threshold concentration for survival of ocean-type salmonids is on the order of $1 \mathrm{~g} / \mathrm{L}$.

Temperature and salinity extremes typically stress fish. Salinity extremes can occur during extreme low-flow conditions, which allow more salt farther up into the estuary. Temperature extremes can occur in the summer over shallow flats and channels during low tides.

Stranding can occur when fish are washed up onto higher ground by waves or ship wakes, or if they are caught for extended periods of time in a shallow pool during an extended low tide. Fisheries biologists have observed stranding of salmonids in the lower Columbia River system.

Entrainment refers to the uptake of fish during dredging. Because dredging occurs primarily in the deepest portions of the channel, bottom-dwelling fish would be more susceptible to being entrained. Surface-oriented fish, such as salmonids, may be less susceptible. 

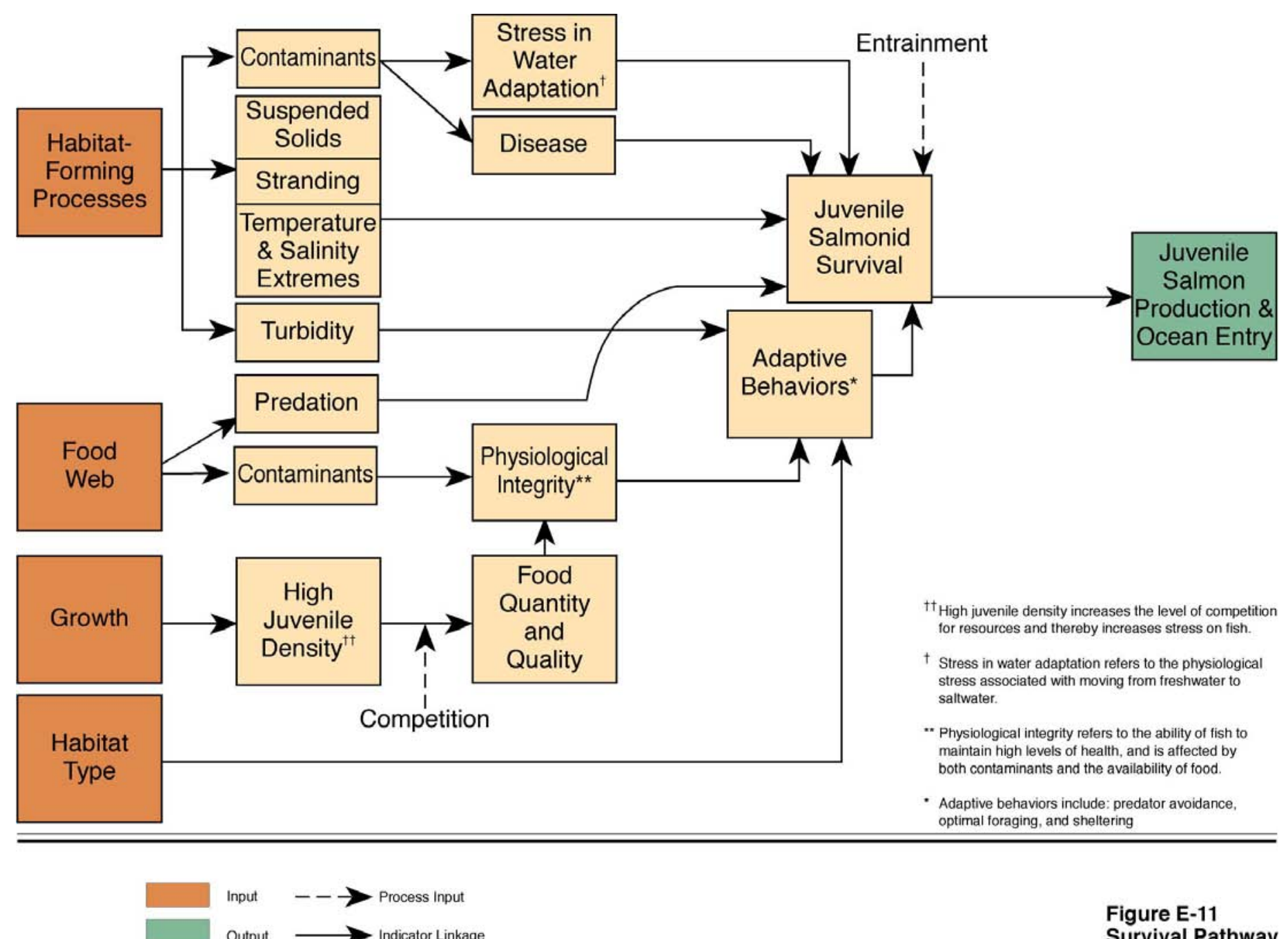

Figure E-11

Output $\longrightarrow$ Indicator Linkage

Survival Pathway

Figure B 10. Survival Pathway. 
Finally, competition among members of the outmigrating population may play a role in survival; however, little is understood or documented regarding the effects of competition in the lower Columbia River.

Adaptive behavior improves the probability that salmonids will survive. The adaptive behaviors of predator avoidance, feeding optimally in the system, and ability to find refuge are all enhanced if fish are healthy. As described in several pathways above, salmonid health depends on physiological integrity, as well as the availability and quality of habitats.

\section{B.7 Pathways and Indicators}

The conceptual model is a way to show the interactions and relationships within a system that, when they are operating properly, help to characterize the system as a whole. This conceptual model for juvenile salmonids consists of several submodels that represent the primary functions of the system. Each of these submodels is composed of several components that link together common relationships associated with maintaining the primary functions. These submodels are the "pathways" in which the components operate for a common function. Each of the components, in turn, may have many states, values, or characteristics that are indicative of the function of the pathway at a particular time; therefore, these components are called "indicators." 
